\title{
Semi-classical analysis of the string theory cigar
}

\author{
Daniel Louis Jafferis and Elliot Schneider \\ Center for the Fundamental Laws of Nature, Harvard University, \\ 17 Oxford Street, Cambridge, MA 02138, U.S.A. \\ E-mail: jafferis@physics.harvard.edu, \\ elliot.andrew.schneider@gmail.com
}

Abstract: We study the semi-classical limit of the reflection coefficient for the $\mathrm{SL}(2, \mathbb{R})_{k} / \mathrm{U}(1) \mathrm{CFT}$. For large $k$, the CFT describes a string in a Euclidean black hole of 2-dimensional dilaton-gravity, whose target space is a cigar with an asymptotically linear dilaton. This sigma-model description is weakly coupled in the large $k$ limit, and we investigate the saddle-point expansion of the functional integral that computes the reflection coefficient. As in the semi-classical limit of Liouville CFT studied in [1], we find that one must complexify the functional integral and sum over complex saddles to reproduce the limit of the exact reflection coefficient. Unlike Liouville, the $\mathrm{SL}(2, \mathbb{R})_{k} / \mathrm{U}(1) \mathrm{CFT}$ admits bound states that manifest as poles of the reflection coefficient. To reproduce them in the semi-classical limit, we find that one must sum over configurations that hit the black hole singularity, but nevertheless contribute to the saddle-point expansion with finite action.

Keywords: Black Holes in String Theory, Conformal Field Theory, Nonperturbative Effects, Sigma Models

ARXiv EPrint: 2004.05223 


\section{Contents}

1 Introduction and overview 1

2 Asymptotic conditions in linear-dilaton backgrounds $\quad 6$

2.1 Asymptotic conditions in free theory 6

$\begin{array}{lll}2.2 & \text { Background-charge operators } & 7\end{array}$

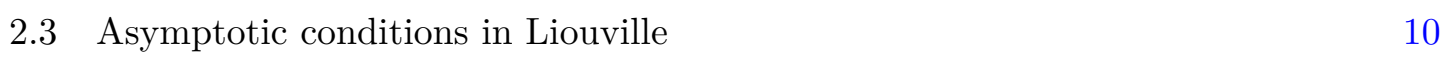

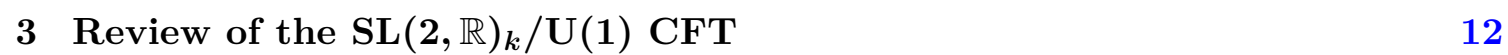

$\begin{array}{lll}3.1 & \text { The cigar sigma-model } & 12\end{array}$

$\begin{array}{lll}3.2 & \text { Operator spectrum } & 13\end{array}$

$\begin{array}{lll}3.3 & \text { Asymptotic conditions in the cigar } & 16\end{array}$

4 Semi-classical limit $\quad 19$

$\begin{array}{ll}\text { 4.1 The reflection coefficient in the large } k \text { limit } & 19\end{array}$

$\begin{array}{ll}4.2 \text { Saddle-point expansion } & 21\end{array}$

4.2.1 Complex quantum mechanics 25

4.2.2 Reflection coefficient on the complex $r$-plane 28

4.2.3 Transmission coefficient on the complex $r$-plane 35

$\begin{array}{lll}4.3 & \text { Bound states and the cigar wrapping saddle } & 37\end{array}$

$\begin{array}{lll}4.4 & \text { Scattering states } & 42\end{array}$

$5 \quad$ Sine-Liouville limit $\quad 44$

$\begin{array}{lll}6 & \text { Discussion } & 48\end{array}$

A Exact solution of the cigar quantum mechanics 49

\section{Introduction and overview}

The $\mathrm{SL}(2, \mathbb{R})_{k} / \mathrm{U}(1) \mathrm{CFT}$ has been a subject of great interest for almost 30 years, since it was shown in [2] that for large $k$ it describes a string in a Euclidean black hole of 2dimensional dilaton-gravity. Much is known about the CFT, thanks in particular to its simple relation to the $\mathrm{SL}(2, \mathbb{R})_{k}$ and $\mathrm{SL}(2, \mathbb{C})_{k} / \mathrm{SU}(2)$ WZW models, which are themselves well-studied CFTs [3-11].

In recent years there has also been renewed interest in the saddle-point expansions of functional integrals, inspired in large part by $[1,12-15]$. In these and related contexts [16$20]$, it has been shown that in order to compute the saddle-point expansion of a functional integral one must, in general, complexify the integral and sum over complex saddles. In particular, in [1] the saddle-point expansions for the two and three-point functions of 
Virasoro primaries $V_{\alpha}(z, \bar{z})$ in Liouville CFT were studied for general complex values of $\alpha$. By comparing to the exact correlation functions, known from [21, 22], the authors of [1] identified the saddles that contribute to the corresponding functional integrals. Even for real values of $\alpha$, the functional integral receives contributions from complex saddle-points. ${ }^{1}$

In this paper, we similarly investigate the saddle-point expansion of the two-point function for the $\mathrm{SL}(2, \mathbb{R})_{k} / \mathrm{U}(1) \mathrm{CFT}$. The exact answer is again known [6, 8, 9, 11], and by comparing to its semi-classical limit we identify the saddles that contribute to the functional integral. We again find that one must sum over complex saddles to reproduce the known result. In fact, as has long been known in Liouville CFT [23] and is also the case in the $\mathrm{SL}(2, \mathbb{R})_{k} / \mathrm{U}(1) \mathrm{CFT}$, the functional integral over real fields for the two-point function is divergent. ${ }^{2}$ Instead, the functional integral for these and related (asymptotic) linear-dilaton backgrounds should in general be defined by an integral over a contour in complexified field space [1]. By identifying the complex saddles that contribute to the functional integral, one may in fact define the appropriate integration cycle by the sum of steepest-descent contours attached to the contributing saddles [1].

The necessity of complexification is of course encountered already in finite-dimensional integrals. For example, when evaluating the asymptotic expansion of a real integral $\int_{\mathcal{C}=\mathbb{R}} \mathrm{d} X e^{-k \tilde{S}[X]}$, one typically continues $\tilde{S}[X]$ to a holomorphic function on the complex $X$-plane, identifies its saddle-points $\tilde{S}^{\prime}\left[X_{n}\right]=0$, constructs the steepest-descent contours $\mathcal{C}_{n}$ attached to each saddle, and deforms the original integration contour into the sum of steepest-descent contours $\mathcal{C}=\sum_{n \in D} \mathcal{C}_{n}$ that is Cauchy-equivalent to the original contour. In the $k \rightarrow \infty$ limit, the integral along a steepest-descent contour $\mathcal{C}_{n}$ is dominated by the contribution from its saddle, $e^{-k \tilde{S}\left[X_{n}\right]}$. Thus, in such favorable circumstances the asymptotic expansion of the original integral is given by the sum $\sum_{n \in D} e^{-k \tilde{S}\left[X_{n}\right]}$ of contributions from the subset of saddles that lie on the deformed integration contour.

One may apply analogous methods to extract the asymptotic expansions of functional integrals $[1,12-15]$. The main complication in the infinite-dimensional case is that it is challenging to derive from first principles which sum of steepest-descent contours is equivalent to the original contour, and therefore which subset of saddles one should sum over in computing the asymptotic expansion [13]. As cited above, however, since the exact result for the $\mathrm{SL}(2, \mathbb{R})_{k} / \mathrm{U}(1) \mathrm{CFT}$ is known, we may take its semi-classical limit and identify the set of saddles that reproduce it.

The $\mathrm{SL}(2, \mathbb{R})_{k}$ WZW model describes a string in $\operatorname{AdS}_{3} \simeq \mathrm{SL}(2, \mathbb{R})$, where $k=l_{\text {AdS }}^{2} / l_{s}^{2}$ sets the AdS length. In global coordinates, $\mathrm{AdS}_{3}$ is a solid cylinder with Lorentzian time running along its length. The $\mathrm{SL}(2, \mathbb{R})_{k} / \mathrm{U}(1)$ coset gauges the time translation isometry, yielding a unitary CFT. At large $k$, it admits a weakly-coupled Lagrangian description given by a sigma-model into a cigar-shaped geometry with an asymptotically linear dilaton, pictured in figure 1 [2]. With the compact coordinate $\theta \sim \theta+2 \pi$ interpreted as Euclidean time, one obtains a two-sided black hole when the geometry is continued to Lorentzian signature [2], with its horizon at $r=0$ where the $\theta$ circle shrinks.

\footnotetext{
${ }^{1}$ Except for a special range of $\alpha$ 's in the case of the three-point function.

${ }^{2} \mathrm{As}$ is the partition function.
} 


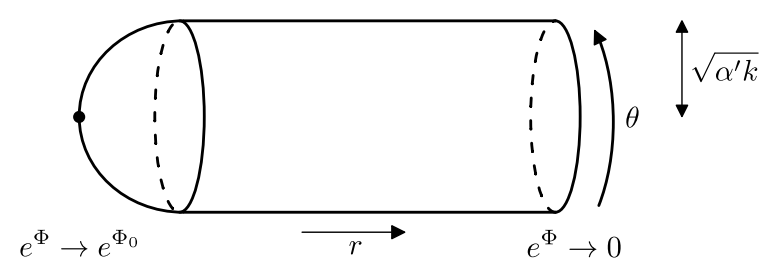

Figure 1. The cigar background. The cigar sigma-model is a weakly-coupled Lagrangian description of the $\mathrm{SL}(2, \mathbb{R})_{k} / \mathrm{U}(1)$ CFT when $k$ is large. For large $r$, the geometry is a cylinder of radius $\sqrt{\alpha^{\prime} k}$, and as $r \rightarrow 0$ the cylinder smoothly caps off. The dilaton is a monotonically decreasing function of $r$. Its maximal value $\Phi_{0}$ is attained at the tip, and at large $r$ it falls off linearly as $-r$. Although a string in the weak-coupling region appears to be able to wind around the cylinder, there is no conserved topological charge because the string can unwind at the tip.

The Virasoro primaries $\mathcal{O}_{j n w}$ of the CFT are labeled by three quantum numbers (cf. eq. (3.9)). $n$ and $w$ are integers, and $j$ is a complex number that is constrained in the normalizable spectrum of the CFT, though we consider its analytic continuation to general complex values. In the asymptotic $r \rightarrow \infty$ region, the cigar approaches a free linear-dilaton $\times S^{1}$ background, and the abstract primaries may be expanded in linear-dilaton $\times S^{1}$ primaries [3]:

$$
\mathcal{O}_{j n w} \stackrel{r \rightarrow \infty}{\longrightarrow}\left(e^{-2(1-j) r}+R(j, n, w) e^{-2 j r}\right) e^{i(n-k w) \theta_{\mathrm{L}}+i(n+k w) \theta_{\mathrm{R}}} .
$$

$\theta_{\mathrm{L}}(z)$ and $\theta_{\mathrm{R}}(\bar{z})$ are the chiral components of the asymptotic circle, $n$ is the momentum number around the circle, and $w$ is (minus) the winding number. $j$ and its reflection $1-j$ are meanwhile momenta along the asymptotic linear-dilaton direction. The reflection coefficient $R(j, n, w)$, which is known exactly (cf. eq. (3.19)) $[6,9,11]$, is the amplitude for a string sent in from the asymptotic region to reflect in the interior and return to infinity.

The normalization chosen in eq. (1.1) is not canonical, and the two-point function of $\mathcal{O}_{j n w}$ and $\mathcal{O}_{j,-n,-w}$ is proportional to $R(j, n, w)$. It is $R$ that we wish to compute by a saddle-point expansion. As an abstract CFT quantity, it characterizes a redundancy in the space of CFT operators $\mathcal{O}_{j n w}$ when analytically continued to the complex $j$-plane; operators labeled by $j$ and $1-j$ are identical, up to rescaling by the reflection coefficient.

The redundancy under $j \rightarrow 1-j$ is a reflection about $j=\frac{1}{2}$. To avoid double-counting operators, one restricts the domain to $\operatorname{Re}(j)>\frac{1}{2}$ or $j \in \frac{1}{2}+i \mathbb{R}_{+}$. This is the $\operatorname{SL}(2, \mathbb{R})_{k} / \mathrm{U}(1)$ version of the Seiberg bound for Liouville CFT [23]. The set $j \in \frac{1}{2}+i \mathbb{R}_{+}$corresponds to delta-function normalizable scattering states on the cigar [3], analogous to the spectrum of Liouville. When $\operatorname{Re}(j)>0$, on the other hand, $e^{-2(1-j) r}$ is exponentially dominant over $e^{-2 j r}$ in eq. (1.1), and the wavefunction of the associated state generically diverges in the asymptotic region. ${ }^{3}$

\footnotetext{
${ }^{3}$ Due to the background-charge contribution from the dilaton, the radial wavefunction at large $r$ differs from eq. (1.1) by an additional factor of $e^{r}$,

$$
\Psi_{j n w}(r) \stackrel{r \rightarrow \infty}{\longrightarrow} e^{-2\left(\frac{1}{2}-j\right) r}+R(j, n, w) e^{-2\left(j-\frac{1}{2}\right) r},
$$

as reviewed in sections 2 and 3 .
} 
At special real values of $j_{N}>\frac{1}{2}$, however, $R\left(j_{N}, n, w\right)$ has simple poles. Then it is the otherwise sub-leading term $e^{-2 j_{N} r}$ that dominates in the asymptotic region, and one obtains normalizable bound states defined as the residue of $\mathcal{O}_{j n w}$ as $j \rightarrow j_{N}[3,24]$. This discrete spectrum of bound states is a remarkable feature of the $\mathrm{SL}(2, \mathbb{R})_{k} / \mathrm{U}(1) \mathrm{CFT}$ that has no analog in Liouville. To reproduce these poles of the reflection coefficient in the saddle-point expansion, we find that we must sum over configurations that hit the singularity of the black hole in the complexified target space. ${ }^{4}$

We are interested in the reflection coefficient for "heavy" operators, for which $j \equiv \frac{k \eta}{2}$ is order $k$, and therefore contributes at the same order as the leading terms in the action in the $k \rightarrow \infty$ limit. We are moreover primarily interested in the pure-winding bound states, for which $n=0$. In this sector, we argue that the only classical solution for $\theta(\rho, \phi)$ that contributes to the saddle-point expansion is the simplest winding solution $\theta=-w \phi$, where $\rho$ and $\phi \sim \phi+2 \pi$ are Euclidean worldsheet cylinder coordinates. Then we show that the integral over $r(\rho, \phi)$ reduces to a quantum mechanics problem in the potential $V(r)=-\frac{1}{2} w^{2} \operatorname{sech}^{2}(r)$, with action (cf. eq. (4.14))

$$
\tilde{S}[r]=\int_{-L}^{L} \mathrm{~d} \rho\left(\frac{1}{2}\left(\frac{\mathrm{d} r}{\mathrm{~d} \rho}\right)^{2}+V(r)\right)-\eta(r(L)+r(-L))+\eta^{2} L .
$$

Here $\rho \in[-L, L]$ is an interval of length $2 L$, which is taken to infinity. The action is defined by this limiting procedure so that the boundary terms $-\eta(r(L)+r(-L))$ insert in the far past and future the operators with $j=\frac{k \eta}{2}$ whose two-point function we wish to compute $[1,22]$. The last term $\eta^{2} L$ is a counterterm that renders the on-shell action finite in the $L \rightarrow \infty$ limit. Using the energy conservation equation in the inverted-potential $-V(r)$, the on-shell action may be written as a contour integral in the $r$-plane:

$$
\tilde{S}[r]=\int_{\mathscr{C}} \mathrm{d} r \sqrt{\eta^{2}+2 V(r)}-\eta(r(L)+r(-L)) .
$$

This quantum mechanics may in fact be solved exactly, as we review in appendix A. There we show that the semi-classical limit of the exact quantum mechanics reflection coefficient reproduces the semi-classical limit of the exact CFT reflection coefficient at order $e^{k}$ (cf. eq. (A.35)). The two differ beginning at order one. Thus, the saddle-point expansion of the reflection coefficient of the CFT in a pure-winding sector reduces to a saddle-point expansion of a quantum mechanics path integral. ${ }^{5}$ Note that the quantum mechanics is defined on a half-line, the radial cigar coordinate being non-negative. Its reflection coefficient may be obtained from the difference of the reflection and transmission coefficients for the quantum mechanics on the full-line.

Our task therefore reduces to computing the saddle-point expansions of the reflection and transmission coefficients for the complexified quantum mechanics on the full $r$-plane.

\footnotetext{
${ }^{4}$ Similar complex contours hitting the black hole singularity were considered in Hartle and Hawking's path integral derivation of Hawking radiation [25].

${ }^{5}$ The same reduction to quantum mechanics occurs in the saddle-point expansion of the Liouville reflection coefficient, though the calculation is presented from a slightly different perspective in [1].
} 
As expected, one must sum over complex solutions, even when $\eta$ is real. ${ }^{6}$ Rather more surprising, however, is that one must also sum over singular configurations that hit the poles of $V(r)$. The potential has double poles at $r=\frac{\pi i}{2}+\pi i \mathbb{Z}$, which coincide with the physical singularities of the Lorentzian black hole in the continued geometry. Though singular, we argue that these trajectories contribute to the functional integral with finite action. In the neighborhood of a pole $z=r-\frac{\pi i}{2}$, the equation of motion is $\frac{\mathrm{d} z}{\mathrm{~d} \rho} \propto \frac{1}{z}$ (cf. eq. (4.37)). The speed-squared of the solution has a $\frac{1}{\rho}$ singularity at the pole, which is integrable up to an ambiguity in its imaginary part by $2 \pi i \mathbb{Z}$. The ambiguity amounts to the choice of deformation of the contour $\mathscr{C}$ in eq. (1.4) along which one evaluates the on-shell action to avoid the pole. Singular saddles were similarly necessary to understand the three-point function in Liouville in [1], and in related contexts in [18].

Thus, one obtains families of saddles with common real part, but whose imaginary parts differ by integer multiples. With these ingredients, we are able to construct contours $\mathscr{C}$ for which the saddle-point expansions reproduce the semi-classical limits of the exact results. The full answer is expected to be a sum over appropriate resummations of the perturbative expansions around each of these saddles. It would be interesting to study whether these perturbative series are Borel resummable. The agreement we find with the factorized $e^{\mathcal{O}(k)} \times e^{\mathcal{O}(1)}$ structure of the large $k$ limit of the exact answer indicates that the one-loop determinants around all of the contributing saddles are equal up to signs, so that their sum with equal weights is not corrected at leading order in $k$. This is not unexpected given that all of the saddles we discuss are related by varying an impact parameter at infinity, and we note that it also applies to the singular saddles.

The outline of the remainder of the paper is as follows. In section 2 we review the formulation of asymptotic conditions in linear-dilaton theories, by which operator insertions in the functional integral may be described by cutting out the neighborhood of the insertion and adding an appropriate boundary term to the action. In section 3 we review the cigar sigma-model background that describes the $\mathrm{SL}(2, \mathbb{R})_{k} / \mathrm{U}(1) \mathrm{CFT}$ at large $k$, the operator spectrum of the CFT, and the associated asymptotic conditions that describe operator insertions in the cigar background. In section 4 we come to the main calculation of the paper, where we show that the large $k$ limit of the exact reflection coefficient of winding operators in the $\mathrm{SL}(2, \mathbb{R})_{k} / \mathrm{U}(1)$ CFT may be reproduced by a saddle-point expansion in complexified field space. Finally, in section 5 we discuss the $k \rightarrow 2$ limit of the $\operatorname{SL}(2, \mathbb{R})_{k} / \mathrm{U}(1)$ CFT, which according to the FZZ duality admits a dual Lagrangian description given by the sine-Liouville background. We argue that the saddle-point expansion in this limit is again given by a sum over complex cycles, and we reproduce the poles of the reflection coefficient in this limit. Appendix A reviews the exact solution of the quantum mechanics that describes the pure-winding sector of the cigar, which is relevant to the calculation of the saddle-point expansion in section 4.

\footnotetext{
${ }^{6}$ Except for the transmission coefficient when $\eta>w$, as discussed in section 4.2.3. In that case a single real saddle is sufficient, corresponding to a particle that rolls over the inverted-potential hill $-V(r)$. For $\eta<w$ there is likewise a real saddle of the reflection coefficient corresponding to a particle that rolls partway up the hill and then rolls back to infinity. But in that case one must also sum over infinitely many complex saddles to reproduce the limit of the exact reflection coefficient.
} 


\section{Asymptotic conditions in linear-dilaton backgrounds}

Before coming to the $\mathrm{SL}(2, \mathbb{R})_{k} / \mathrm{U}(1) \mathrm{CFT}$, in this section we review some aspects of linear-dilaton theories that will be important in what follows, especially the formulation of "asymptotic conditions." These provide a convenient description of operator insertions in the functional integral via boundary modifications of the action. ${ }^{7}$

\subsection{Asymptotic conditions in free theory}

A local operator insertion in a functional integral produces a delta-function source in the equations of motion, which, semi-classically, requires that the saddles behave as the associated Green function in the neighborhood of the insertion. The resulting saddles are singular at the insertion point. With an asymptotic condition, this singular behavior is regulated by cutting out the neighborhood of the insertion from the worldsheet and introducing an appropriate boundary action there. The boundary term is chosen such that the boundary equations of motion impose the required Green function behavior. In the limit that the cut-out neighborhood shrinks away, the functional integral defined by the action with boundary reproduces the functional integral with the operator insertion.

To understand how to apply this procedure in practice, consider first the free theory of a non-compact boson $X(z, \bar{z})$ with a linear-dilaton ${ }^{8} \Phi(X)=-Q X$. The action in locally flat complex coordinates is

$$
S=\frac{1}{2 \pi \alpha^{\prime}} \int \mathrm{d}^{2} z \partial X \bar{\partial} X+\cdots,
$$

up to boundary terms due to the dilaton, which we will account for shortly. The Virasoro primaries may be written as $V_{\alpha}(z, \bar{z})=e^{-2 \alpha X(z, \bar{z})}$, where we will consider the analytic continuation of $\alpha$ to general complex values. They are scalars, of conformal weights

$$
h_{\alpha}=\bar{h}_{\alpha}=\alpha^{\prime} \alpha(Q-\alpha)
$$

with respect to the holomorphic stress tensor

$$
T(z)=-\frac{1}{\alpha^{\prime}}(\partial X)^{2}-Q \partial^{2} X
$$

and its anti-holomorphic counterpart. The stress tensor satisfies the Virasoro algebra with central charge $c_{X}=1+6 \alpha^{\prime} Q^{2}$.

Note that the conformal weights are symmetric under reflection about $\alpha=\frac{Q}{2}: h_{\alpha}=$ $h_{Q-\alpha}$. In the free theory, $\alpha$ and $Q-\alpha$ label independent operators, though in the interacting theories of interest they will in fact correspond to the same operator. Note also that the weights are real when $\alpha \in \mathbb{R}$ or $\alpha \in \frac{Q}{2}+i \mathbb{R}$, which are referred to as the real and complex branches of operators. On the complex branch, $h_{\alpha}=\alpha^{\prime}|\alpha|^{2} \geq \alpha^{\prime} \frac{Q^{2}}{4}$ is always positive. On

\footnotetext{
${ }^{7}$ This section is included to establish conventions and keep the paper self-contained. Experienced readers may wish to go directly to section 3 .

${ }^{8}$ We take $Q$ real and positive, such that the effective string coupling $e^{\Phi(X)}$ decays at $X \rightarrow \infty$ and diverges at $X \rightarrow-\infty$, which we refer to as the weak and strong coupling regions.
} 
the real branch, the weight is only positive in the window $0<\alpha<Q$, its maximal value coinciding with the minimal weight on the complex branch.

Inserting $V_{\alpha}\left(z^{\prime}, \bar{z}^{\prime}\right)$ in the functional integral,

$$
\begin{aligned}
\int D X & e^{-S} V_{\alpha}\left(z^{\prime}, \bar{z}^{\prime}\right) \\
& =\int D X \exp \left\{-\frac{1}{2 \pi \alpha^{\prime}} \int \mathrm{d}^{2} z\left(\partial X \bar{\partial} X+4 \pi \alpha^{\prime} \alpha \delta\left(z-z^{\prime}, \bar{z}-\bar{z}^{\prime}\right) X(z, \bar{z})\right)\right\},
\end{aligned}
$$

introduces a source term in the bulk equation of motion,

$$
\partial \bar{\partial} X=2 \pi \alpha^{\prime} \alpha \delta\left(z-z^{\prime}, \bar{z}-\bar{z}^{\prime}\right) .
$$

Recalling the Green function for the 2-dimensional wave equation, $\partial \bar{\partial} \log (z \bar{z})=2 \pi \delta(z, \bar{z})$, we find that in the neighborhood of the insertion point on the worldsheet the solution of the equation of motion is

$$
X(z, \bar{z}) \stackrel{\left|z-z^{\prime}\right| \rightarrow 0}{\longrightarrow} 2 \alpha^{\prime} \alpha \log \left|z-z^{\prime}\right|+\mathcal{O}(1)
$$

or $X(\rho, \phi) \stackrel{\rho \rightarrow-\infty}{\longrightarrow} 2 \alpha^{\prime} \alpha \rho+\mathcal{O}(1)$ in local cylinder coordinates $z-z^{\prime} \equiv e^{\rho+i \phi}$. Thus, the operator insertion requires that the solution is asymptotically linear in $\rho$, with $\partial_{\rho} X \rightarrow 2 \alpha^{\prime} \alpha$ as $\rho \rightarrow-\infty$.

Let us therefore cut out a small disk $d_{\varepsilon}$ of radius $\left|z-z^{\prime}\right|=\varepsilon$ surrounding the insertion point and deform the action by a boundary term [1, 22]:

$$
S(\varepsilon)=S+2 \alpha \int_{\partial d_{\varepsilon}} \frac{\mathrm{d} \phi}{2 \pi} X-2 \alpha^{\prime} \alpha^{2} \log (\varepsilon),
$$

where $\mathrm{d} \phi=\frac{1}{2 i}\left(\frac{\mathrm{d} z}{z-z^{\prime}}-\frac{\mathrm{d} \bar{z}}{\bar{z}-\bar{z}^{\prime}}\right)$. Then the boundary variation $-\frac{1}{2 \pi \alpha^{\prime}} \int_{\partial d_{\varepsilon}} \mathrm{d} \phi \delta X \partial_{\rho} X$ of $S$ and the variation of the boundary term $2 \alpha \int_{\partial d_{\varepsilon}} \frac{\mathrm{d} \phi}{2 \pi} \delta X$ yield the desired boundary equation of motion,

$$
\left.\partial_{\rho} X\right|_{\rho=\log (\varepsilon)}=2 \alpha^{\prime} \alpha .
$$

In the limit $\varepsilon \rightarrow 0$, one expects the functional integral weighted by the deformed action $e^{-S(\varepsilon)}$ to reproduce the functional integral weighted by $e^{-S} e^{-2 \alpha X\left(z^{\prime}, \bar{z}^{\prime}\right)}$. The counterterm $-2 \alpha^{\prime} \alpha^{2} \log (\varepsilon)$ is included to render the on-shell action finite.

\subsection{Background-charge operators}

Even in the absense of any operator insertions, however, the action eq. (2.1) is supplemented by boundary terms due to the non-trivial dilaton. To understand these terms, consider the more general sigma-model action on a worldsheet $\Sigma$ with metric $h$,

$$
\begin{aligned}
S[X ; h]= & \frac{1}{4 \pi \alpha^{\prime}} \int_{\Sigma} \mathrm{d}^{2} \sigma \sqrt{h} h^{a b} \partial_{a} X \partial_{b} X \\
& -Q\left(\frac{1}{4 \pi} \int_{\Sigma} \mathrm{d}^{2} \sigma \sqrt{h} \mathcal{R} X+\frac{1}{2 \pi} \int_{\partial \Sigma} \mathrm{d} \phi \sqrt{\gamma} \mathcal{K} X\right) .
\end{aligned}
$$


Here, $\mathcal{R}$ is the scalar curvature of $h, \mathcal{K}$ is the trace of the extrinsic curvature of the boundary (if present), $\gamma$ is the induced metric of the boundary, and $\mathrm{d} \phi \sqrt{\gamma}$ is the induced volume form.

The sigma-model is Weyl-invariant up to a field-independent anomaly, provided that $X$ simultaneously transforms as a Goldstone boson,

$$
S\left[X+\alpha^{\prime} Q \omega ; e^{2 \omega} h\right]=S[X ; h]-S\left[-\alpha^{\prime} Q \omega ; h\right] .
$$

The dilaton violates the target translation symmetry of the kinetic term, $S[X+\varepsilon ; h]=$ $S[X ; h]-\varepsilon Q \chi$, with $\chi$ the Euler characteristic of $\Sigma$, which implies the anomalous conservation law

$$
\sum_{j} \alpha_{j}=\frac{1}{2} Q \chi
$$

for a correlation function of operators $\prod_{j} V_{\alpha_{j}}$. The bulk equation of motion,

$$
\nabla^{2} X=-\frac{1}{2} \alpha^{\prime} Q \mathcal{R}[h]
$$

likewise reflects the anomalous conservation of the currents $\partial X$ and $\bar{\partial} X$.

We are interested in the theory at string tree-level, for which $\Sigma$ has the topology of a sphere and $\chi=2$. Eq. (2.1) was written in locally flat coordinates $\mathrm{d} s^{2}=\mathrm{d} z \mathrm{~d} \bar{z}$, and would follow from eq. (2.9) by discarding the curvature terms. Globally, however, there does not exist a flat metric on the sphere. In particular, the coordinate $z$ does not cover the neighborhood of the point-at-infinity, and the "flat" metric is singular there: $\mathrm{d} s^{2}=\frac{\mathrm{d} u \mathrm{~d} \bar{u}}{(u \bar{u})^{2}}$, with a local coordinate $u=\frac{1}{z}$. This singularity contributes a delta-function source of curvature, $\mathcal{R}=16 \pi(u \bar{u})^{2} \delta(u, \bar{u})=16 \pi \delta\left(z-z_{\infty}, \bar{z}-\bar{z}_{\infty}\right)$, as required to reproduce the Euler characteristic of the sphere.

To avoid this singular behavior, one could choose instead the round metric, $\mathrm{d} s^{2}=$ $\frac{4}{(1+z \bar{z})^{2}} \mathrm{~d} z \mathrm{~d} \bar{z}$, for which $\mathcal{R}=2$ is a constant. However, the dilaton term then produces a linear potential, which is slightly awkward. It is instead common practice to work with the plane metric $\mathrm{d} z \mathrm{~d} \bar{z}$ or the cylinder metric $\frac{\mathrm{d} z \mathrm{~d} \bar{z}}{z \bar{z}}$, which are related to the round metric by singular Weyl transformations that push all the curvature of the sphere to the pointat-infinity or the two ends of the cylinder.

With the plane metric, the effect of the curvature singularity is to shift eq. (2.1) by $-2 Q X\left(z_{\infty}, \bar{z}_{\infty}\right)$, which may be thought of as an insertion of $V_{-Q}\left(z_{\infty}, \bar{z}_{\infty}\right)$ at the point-atinfinity. Thus, one can study the linear-dilaton background in flat coordinates, provided that one keeps track of this so-called background-charge operator. It inserts a fixed source in the equation of motion,

$$
\partial \bar{\partial} X=-2 \pi \alpha^{\prime} Q \delta\left(z-z_{\infty}, \bar{z}-\bar{z}_{\infty}\right)
$$

again demonstrating the anomalous conservation of the $\mathfrak{u}_{1}$ currents in the presence of the linear-dilaton, and imposing the asymptotic condition

$$
X(z, \bar{z}) \stackrel{|z| \rightarrow \infty}{\longrightarrow} 2 \alpha^{\prime} Q \log |z|+\mathcal{O}(1)
$$


The Green function $2 \alpha^{\prime} \sum_{j} \alpha_{j} \log \left|z-z_{j}\right|$ in the presence of operator insertions $\prod_{j} V_{\alpha_{j}}\left(z_{j}, \bar{z}_{j}\right)$ satisfies the asymptotic condition only provided

$$
\sum_{j} \alpha_{j}=Q
$$

reproducing eq. (2.11). Note also that the 1-point function of the operator $V_{Q}(z, \bar{z})$ is not required to vanish, which is compatible with conformal symmetry because $h_{Q}=\bar{h}_{Q}=0$.

By the earlier discussion, the background-charge insertion at the point-at-infinity may be replaced by excising its neighborhood and introducing a boundary term as in eq. (2.7). The action for the linear-dilaton on the plane is therefore given by the $R \rightarrow \infty$ limit of $[1,22,26]$

$$
S=\frac{1}{2 \pi \alpha^{\prime}} \int_{D_{R}} \mathrm{~d}^{2} z \partial X \bar{\partial} X-2 Q \int_{\partial D_{R}} \frac{\mathrm{d} \phi}{2 \pi} X+2 \alpha^{\prime} Q^{2} \log (R),
$$

with $D_{R}$ a disk of radius $R$. Note that the prescription amounts to cutting out the source and doubling the extrinsic curvature term in eq. (2.9) on the resulting boundary, which ensures that $2 \times \frac{1}{2 \pi} \int_{\partial \Sigma} \mathrm{d} \phi \sqrt{\gamma} \mathcal{K}=2$ produces the Euler characteristic of the sphere rather than the disk. As before, one may introduce additional insertions $V_{\alpha_{j}}\left(z_{j}, \bar{z}_{j}\right)$ by cutting out disks at $\left(z_{j}, \bar{z}_{j}\right)$ and including additional boundary terms as in eq. (2.7).

For the most part, we will actually work on the cylinder rather than the plane, corresponding to the metric $\mathrm{d} s^{2}=\frac{\mathrm{d} z \mathrm{~d} \bar{z}}{z \bar{z}}$. The cylinder metric is singular at both its ends, $\mathcal{R}=8 \pi z \bar{z}\left(\delta(z, \bar{z})+\delta\left(z-z_{\infty}, \bar{z}-\bar{z}_{\infty}\right)\right)$, and the background-charge is now split symmetrically between them with insertions $V_{-Q / 2}(0)$ and $V_{-Q / 2}\left(z_{\infty}, \bar{z}_{\infty}\right)$. The equation of motion becomes

$$
\partial \bar{\partial} X=-\pi \alpha^{\prime} Q\left(\delta(z, \bar{z})+\delta\left(z-z_{\infty}, \bar{z}-\bar{z}_{\infty}\right)\right),
$$

and the asymptotic conditions are

$$
X(\rho, \phi) \stackrel{\rho \rightarrow \pm \infty}{\longrightarrow} \pm \alpha^{\prime} Q \rho+\mathcal{O}(1)
$$

where $z=e^{\rho+i \phi}$. The action is given by the $L \rightarrow \infty$ limit of

$$
S=\frac{1}{4 \pi \alpha^{\prime}} \int_{-L}^{L} \mathrm{~d} \rho \int_{0}^{2 \pi} \mathrm{d} \phi\left(\left(\partial_{\rho} X\right)^{2}+\left(\partial_{\phi} X\right)^{2}\right)-Q \int_{0}^{2 \pi} \frac{\mathrm{d} \phi}{2 \pi}\left(\left.X\right|_{\rho=L}+\left.X\right|_{\rho=-L}\right)+\alpha^{\prime} Q^{2} L .
$$

Suppose a primary $e^{-2 \alpha X}$ is inserted in the far past on the cylinder. The asymptotic condition is

$$
X(\rho, \phi) \stackrel{\rho \rightarrow-\infty}{\longrightarrow}-2 \alpha^{\prime}\left(\frac{Q}{2}-\alpha\right) \rho+\mathcal{O}(1)
$$

Note that for $\operatorname{Re}(\alpha)<\frac{Q}{2}$, the asymptotic condition sends $X$ to the weak-coupling region, whereas for $\operatorname{Re}(\alpha)>\frac{Q}{2}$ it is mapped to the strong-coupling region. Comparing to the usual mode-expansion $X=X_{0}-i \alpha^{\prime} P_{0} \rho+\cdots$, one finds that the operator insertion $e^{-2 \alpha X}$ prepares a state on the cylinder of momentum $P_{0}=-2 i\left(\frac{Q}{2}-\alpha\right)$. Note that the reflection $\alpha \rightarrow Q-\alpha$ flips the sign of $P_{0}$. 
The zero-mode wavefunction $e^{i P_{0} X_{0}}$ of the state prepared by $e^{-2 \alpha X}$ is then

$$
\Psi_{\alpha}\left(X_{0}\right)=e^{2\left(\frac{Q}{2}-\alpha\right) X_{0}} .
$$

It differs from $e^{-2 \alpha X}$ by the background-charge operator $e^{Q X}$, which is fixed on the end of the cylinder. More generally, the target space string-frame effective action is multiplied by an overall factor of $e^{-2 \Phi}$. Extracting the target space wavefunction from $e^{-2 \Phi}\left(\nabla_{X} \tilde{\Psi}(X)\right)^{2}$ requires rescaling $\tilde{\Psi}(X) \rightarrow \Psi(X)=e^{-\Phi} \tilde{\Psi}(X)$. For the linear-dilaton, the necessary factor is again $e^{-\Phi}=e^{Q X}$.

For $\alpha \in \frac{Q}{2}+i \mathbb{R}$, the zero-mode wavefunction is oscillatory and delta-function normalizable. Otherwise it is non-normalizable, exponentially diverging either at $X \rightarrow \infty$ for $\operatorname{Re}(\alpha)<\frac{Q}{2}$, or $X \rightarrow-\infty$ for $\operatorname{Re}(\alpha)>\frac{Q}{2}$, in correspondence with the sign of the asymptotic condition.

\subsection{Asymptotic conditions in Liouville}

The above discussion was confined to the free linear-dilaton theory. However, the free linear-dilaton is not a unitary CFT. ${ }^{9}$ As a string background it is clearly ill-behaved because the string coupling $e^{\Phi}$ diverges exponentially as $X \rightarrow-\infty$. This pathological behavior may be regulated by turning on a potential barrier $\mu e^{-2 b_{\mathrm{L}} X}$, with $\mu>0$ and $\operatorname{Re}\left(b_{\mathrm{L}}\right)>0$, that suppresses string configurations that extend too deeply into the strong-coupling region. $b_{\mathrm{L}}$ is fixed by demanding that the potential is marginal, $b_{\mathrm{L}}\left(Q-b_{\mathrm{L}}\right)=1$. The result is the Liouville CFT, with bulk action ${ }^{10}$

$$
S=\frac{1}{2 \pi} \int \mathrm{d}^{2} z\left(\partial X \bar{\partial} X+\pi \mu e^{-2 b_{\mathrm{L}} X}\right)+\cdots
$$

Much of the preceding machinery of the free theory continues to apply, with a few caveats that we describe now. The cigar CFT, discussed in the next section, will be closely analogous.

As $X \rightarrow \infty$, the Liouville potential vanishes and the free linear-dilaton is recovered. One therefore again has scattering solutions of the zero-mode quantum mechanics that behave as plane waves $e^{ \pm i P_{0} X_{0}}$ in the free-field region. They are no longer independent, however. The solutions which decay under the potential in the strong-coupling region behave as linear combinations of incoming and reflected waves in the free-field region, $\Psi\left(X_{0}\right) \stackrel{X_{0} \rightarrow \infty}{\longrightarrow} e^{i P_{0} X_{0}}+R\left(P_{0}\right) e^{-i P_{0} X}$, with $R\left(P_{0}\right)$ the reflection coefficient.

In the CFT, one correspondingly has operators $V_{\alpha}(z, \bar{z})$ behaving asymptotically as ${ }^{11}$

$$
V_{\alpha} \stackrel{X \rightarrow \infty}{\longrightarrow} e^{-2 \alpha X}+R(\alpha) e^{-2(Q-\alpha) X}
$$

\footnotetext{
${ }^{9}$ The operators which map to delta-function normalizable states have $\alpha \in \frac{Q}{2}+i \mathbb{R}$, but the OPE generates operators with $\alpha$ outside this range.

${ }^{10} \mathrm{We}$ set $\alpha^{\prime}=1$ in this subsection to be consistent with the standard convention in Liouville.

${ }^{11}$ With this choice of normalization, the 2-point function of Liouville primaries is proportional to $R(\alpha)$. A canonically normalized 2-point function is obtained by rescaling the primaries by $R(\alpha)^{-1 / 2}$.
} 
The exact reflection coefficient is [21, 22]

$$
R(\alpha)=-\left(\pi \mu \frac{\Gamma\left(b_{\mathrm{L}}^{2}\right)}{\Gamma\left(1-b_{\mathrm{L}}^{2}\right)}\right)^{\frac{2}{b_{\mathrm{L}}}\left(\frac{Q}{2}-\alpha\right)} \frac{\Gamma\left(1-\frac{2}{b_{\mathrm{L}}}\left(\frac{Q}{2}-\alpha\right)\right)}{\Gamma\left(1+\frac{2}{b_{\mathrm{L}}}\left(\frac{Q}{2}-\alpha\right)\right)} \frac{\Gamma\left(1-2 b_{\mathrm{L}}\left(\frac{Q}{2}-\alpha\right)\right)}{\Gamma\left(1+2 b_{\mathrm{L}}\left(\frac{Q}{2}-\alpha\right)\right)} .
$$

It satisfies $R(\alpha) R(Q-\alpha)=1$. Whereas $\alpha$ and $Q-\alpha$ labeled independent operators of identical conformal weights in the free theory, they now label two components of the same operator due to reflection off the potential. One therefore labels Liouville operators by $\alpha$ satisfying $\operatorname{Re}(\alpha) \leq \frac{Q}{2}$, and moreover $\operatorname{Im}(\alpha)>0$ if $\operatorname{Re}(\alpha)=\frac{Q}{2}$, in order to avoid doublecounting. With this convention, $e^{-2 \alpha X}$ is the exponentially dominant term at infinity, except when $\operatorname{Re}(\alpha)=\frac{Q}{2}$, in which case neither term dominates the other. It is impossible to have an operator that asymptotes to $e^{-2 \alpha X}$ with $\operatorname{Re}(\alpha)>\frac{Q}{2}$ because it is sub-dominant to its reflection $e^{-2(Q-\alpha) X}$, and both terms are required to obtain a non-singular solution in the interior [23]. Equivalently, one may allow all values of $\alpha$, in which case the CFT operators $V_{\alpha}$ and $V_{Q-\alpha}$ are identical up to rescaling by the reflection coefficient, $V_{\alpha}=R(\alpha) V_{Q-\alpha}$. The complex and real branches of operators with non-negative conformal weights are labeled by $\alpha \in \frac{Q}{2}+i \mathbb{R}_{+}$and $\alpha \in\left[0, \frac{Q}{2}\right]$.

Consider the worldsheet neighborhood of an operator insertion $V_{\alpha}\left(z^{\prime}, \bar{z}^{\prime}\right)$. Suppose $\operatorname{Re}(\alpha)<\frac{Q}{2}$, such that operator at large $X$ is dominated by $e^{-2 \alpha X}$, as in the free theory. If one further requires $\operatorname{Re}(\alpha)<0$, then the free-field Green function eq. (2.6) remains a self-consistent solution of the Liouville equation of motion, since it maps the neighborhood of the insertion to the free-field region where the potential is sub-leading. As before, one can cut out the insertion and replace it with the boundary action eq. (2.7).

The same considerations as in the free theory require the asymptotic conditions eq. (2.14) on the plane or eq. (2.18) on the cylinder. These likewise map $X \rightarrow \infty$ where the potential is sub-leading, and the free-field results remain consistent.

Inserting $V_{\alpha}$ with $\operatorname{Re}(\alpha)<\frac{Q}{2}$ in the far past on the cylinder imposes the same asymptotic condition as before, eq. (2.20). Indeed, $X$ is sent to the free-field region when $\operatorname{Re}(\alpha)<\frac{Q}{2}$. One may similarly describe a complex branch insertion by shifting $\alpha \rightarrow \alpha-\varepsilon$ with a small regulator $\varepsilon>0$, such that the asymptotic condition again sends $\operatorname{Re}(X) \rightarrow \infty$.

The zero-mode wavefunction for the state prepared by inserting $V_{\alpha}$ in the far past behaves at large $X$ as

$$
\Psi_{\alpha}\left(X_{0}\right) \stackrel{X_{0} \rightarrow \infty}{\longrightarrow} e^{2\left(\frac{Q}{2}-\alpha\right) X_{0}}+R(\alpha) e^{-2\left(\frac{Q}{2}-\alpha\right) X_{0}} .
$$

On the complex branch it is oscillatory and delta-function normalizable, corresponding to a scattering state with asymptotic momentum $2 \operatorname{Im}(\alpha)$, while on the real branch it is exponentially divergent at weak-coupling and therefore non-normalizable. The Hilbert space of normalizable states is then spanned by the complex branch $\alpha \in \frac{Q}{2}+i \mathbb{R}_{+}[23]$. 
With insertions of $V_{\alpha}$ at both ends of the cylinder, one obtains the following action

$$
\begin{aligned}
S_{\alpha}= & \frac{1}{4 \pi} \int_{-L}^{L} \mathrm{~d} \rho \int_{0}^{2 \pi} \mathrm{d} \phi\left(\left(\partial_{\rho} X\right)^{2}+\left(\partial_{\phi} X\right)^{2}+4 \pi \mu e^{-2 b_{\mathrm{L}} X}\right) \\
& -2\left(\frac{Q}{2}-\alpha\right) \int_{0}^{2 \pi} \frac{\mathrm{d} \phi}{2 \pi}\left(\left.X\right|_{\rho=L}+\left.X\right|_{\rho=-L}\right)+4\left(\frac{Q}{2}-\alpha\right)^{2} L,
\end{aligned}
$$

whose functional integral computes the Liouville reflection coefficient $R(\alpha)$ in the limit $L \rightarrow \infty$. The saddle-point expansion of this integral in the semi-classical $\left(b_{\mathrm{L}} \rightarrow 0\right)$ limit was computed in [1], and matched to the limit of the exact reflection coefficient.

Our objective in sections 3 and 4 is to treat the large $k$ limit of the $\operatorname{SL}(2, \mathbb{R})_{k} / \mathrm{U}(1)$ CFT similarly.

\section{Review of the $\mathrm{SL}(2, \mathbb{R})_{k} / \mathrm{U}(1) \mathrm{CFT}$}

The $\mathrm{SL}(2, \mathbb{R})_{k} / \mathrm{U}(1) \mathrm{CFT}$ is a coset of the $\mathrm{SL}(2, \mathbb{R})_{k}$ WZW model. The latter describes a string propagating ${ }^{12}$ in Lorentzian $\mathrm{AdS}_{3}=\mathrm{SL}(2, \mathbb{R})$, where the WZW level $k$ sets the AdS-length, $l_{\mathrm{AdS}}^{2}=k l_{\mathrm{s}}^{2}$. AdS $\mathrm{A}_{3}$ may be described as a solid cylinder, and the coset is defined by gauging the timelike isometry along its length, producing a target space of Euclidean signature. Its central charge is

$$
c=\frac{3 k}{k-2}-1
$$

where $k$ is a real number greater than 2 , which need not be an integer.

We will primarily be interested ${ }^{13}$ in the semi-classical limit of the CFT at large $k$, which describes a string propagating in a 2-dimensional Euclidean black hole [2]. In this section we review the Lagrangian description of the CFT in this limit, given by the cigar sigma-model background. Then we review the operator spectrum of the CFT and the associated asymptotic conditions in the cigar description. Finally, we write the action for the 2-point function of primaries on the cylinder, which we will use in section 4 to compute the saddle-point expansion of the reflection coefficient.

\subsection{The cigar sigma-model}

For large $k$, the coset admits a Lagrangian description given by the following sigma-model background:

$$
\begin{aligned}
\mathrm{d} s^{2} & =\alpha^{\prime} k\left(\mathrm{~d} r^{2}+\tanh ^{2}(r) \mathrm{d} \theta^{2}\right) \\
\Phi & =-\log \cosh (r)+\Phi_{0} .
\end{aligned}
$$

The action on a closed worldsheet $\Sigma$ is

$$
S=\frac{k}{4 \pi} \int_{\Sigma} \mathrm{d}^{2} \sigma \sqrt{h}\left\{(\nabla r)^{2}+\tanh ^{2}(r)(\nabla \theta)^{2}+\frac{1}{k} \mathcal{R}[h]\left(\Phi_{0}-\log \cosh r\right)\right\}
$$

\footnotetext{
${ }^{12} \mathrm{By} \mathrm{AdS}_{3}=\mathrm{SL}(2, \mathbb{R})$ we mean the simply-connected covering-space.

${ }^{13}$ The $k \rightarrow 2$ limit of the CFT is also interesting, and we discuss it briefly in section 5 .
} 
with equations of motion

$$
\begin{aligned}
\nabla^{2} r-\tanh (r) \operatorname{sech}^{2}(r)(\nabla \theta)^{2}+\frac{1}{2 k} \mathcal{R}[h] \tanh (r) & =0 \\
\nabla^{2} \theta+2 \operatorname{sech}(r) \operatorname{csch}(r) h^{a b} \nabla_{a} r \nabla_{b} \theta & =0 .
\end{aligned}
$$

The target space has the topology of a disk, with coordinates $r \in[0, \infty)$ and $\theta \sim \theta+2 \pi$. The dilaton is a monotonically decreasing function of $r$, with the constant $\Phi_{0}$ setting its maximal value at the origin: $\left.\Phi\right|_{r=0}=\Phi_{0}$. In that neighborhood the geometry is simply $\mathbb{R}^{2}$ in polar coordinates:

$$
\begin{aligned}
\mathrm{d} s^{2} & =\alpha^{\prime} k\left(\mathrm{~d} r^{2}+r^{2} \mathrm{~d} \theta^{2}\right)+\mathcal{O}\left(r^{3}\right) \\
\Phi & =\Phi_{0}-\frac{1}{2} r^{2}+\mathcal{O}\left(r^{3}\right) .
\end{aligned}
$$

At large $r$, on the other hand, the geometry approaches a cylinder of radius $\sqrt{\alpha^{\prime} k}$, with a linearly decreasing dilaton along its length:

$$
\begin{aligned}
\mathrm{d} s^{2} & =\alpha^{\prime} k\left(\mathrm{~d} r^{2}+\mathrm{d} \theta^{2}\right)+\mathcal{O}\left(e^{-2 r}\right) \\
\Phi & =-r+\mathcal{O}(1) .
\end{aligned}
$$

The target space therefore resembles a cigar, with its asymptotic cylinder at large $r$ and its tip at $r=0$, where the $\theta$ circle shrinks to a point, as pictured in figure 1 .

For large $k$ the cigar is large and weakly-curved,

$$
\mathcal{R}=\frac{4}{\cosh ^{2}(r)} \frac{1}{\alpha^{\prime} k},
$$

and the sigma-model is weakly-coupled in the $\alpha^{\prime}$ sense. Meanwhile, the string coupling $e^{\Phi}$ attains its maximal value $e^{\Phi_{0}}$ at the tip of the cigar and decays to zero at large $r$. The parameter $\Phi_{0}$ is a modulus of the theory. It reflects the usual freedom to shift the dilaton by a constant, the only effect being to shift the action by $\Phi_{0} \chi$, with $\chi$ the Euler characteristic of $\Sigma$.

The rather exotic coupling to curvature represented by the dilaton eq. (3.2b) is required to satisfy the 1-loop beta function equation [2]

$$
\beta_{I J}(G)=\alpha^{\prime}\left(R_{I J}+2 \nabla_{I} \nabla_{J} \Phi\right)+\mathcal{O}\left(\alpha^{\prime 2}\right)=\mathcal{O}\left(\alpha^{\prime 2}\right),
$$

which implies conformal invariance of the sigma-model to leading order in the large $k$ limit.

\subsection{Operator spectrum}

Although the above sigma-model is a valid description of the coset only at large $k$, the exact spectrum of the CFT is known in light of its relation to the $\operatorname{SL}(2, \mathbb{R})_{k}$ WZW model via the coset construction $[3,4,10]$. The Virasoro primaries $\mathcal{O}_{j n w}(z, \bar{z})$ of the coset are labeled by integers $n$ and $w$ and a complex number $j$, taking the following values:

$$
\begin{aligned}
j & =\frac{1}{2}+i s, & s \in \mathbb{R}_{+} \\
j_{N} & =\frac{k|w|-|n|}{2}-N \in\left(\frac{1}{2}, \frac{k-1}{2}\right), & N \in \mathbb{N} .
\end{aligned}
$$


We refer to these two sets as the complex and real branches of primaries based on the value of $j$. As in the free linear-dilaton and Liouville theories reviewed in the previous section, the complex branch primaries correspond to scattering states on the cigar with momentum proportional to $s$. On the other hand, the real branch primaries with $j=j_{N}$ correspond to bound states living at the tip of the cigar. One may also consider real branch operators where $j$ is not valued in this discrete set, which map to non-normalizable states. The integers $n$ and $w$, meanwhile, correspond to the momentum and winding numbers around the asymptotic cylinder at large $r$.

Note that on the complex branch the value of $j$ is independent of the integers $n$ and $w$, whereas on the real branch $n$ and $w$ determine the allowed values of $j$ up to shifts by the natural number $N$, constrained to lie within the interval $\frac{1}{2}<j<\frac{k-1}{2}$. That lower-bound implies there may only exist real branch primaries with $k|w|-|n|>1$. In particular, there are none with $w=0$.

These primaries carry conformal weights

$$
h_{j n w}=-\frac{j(j-1)}{k-2}+\frac{(n-k w)^{2}}{4 k}, \quad \bar{h}_{j n w}=-\frac{j(j-1)}{k-2}+\frac{(n+k w)^{2}}{4 k} .
$$

Note that the quantity $-j(j-1)$ is a real number not only on the real branch, but also on the complex branch where $-j(j-1)=\frac{1}{4}+s^{2}$. On the real branch, it is non-negative for $\frac{1}{2} \leq j \leq 1$ and negative thereafter, its maximal value coinciding with the minimal value on the complex branch. The total conformal weight is non-negative, however.

Since the sigma-model reduces to the free linear-dilaton $\times S^{1}$ background at large $r$, the abstract primaries $\mathcal{O}_{j n w}$ may be expanded in free-field primaries in that limit. To compare with the formulas from the previous section, one may define a canonically normalized field $\hat{r} \equiv \sqrt{\alpha^{\prime} k} r$, and likewise $\hat{\theta}=\sqrt{\alpha^{\prime} k} \theta$, in terms of which the asymptotic background eq. (3.6) is

$$
\begin{gathered}
\mathrm{d} s^{2} \stackrel{\hat{r} \rightarrow \infty}{\longrightarrow} \mathrm{d} \hat{r}^{2}+\mathrm{d} \hat{\theta}^{2} \\
\Phi \stackrel{\hat{r} \rightarrow \infty}{\longrightarrow}-Q \hat{r},
\end{gathered}
$$

with

$$
Q=\frac{1}{\sqrt{\alpha^{\prime} k}}
$$

Note that $Q$ goes to zero in the large $k$ limit, in contrast to Liouville where $Q \stackrel{b \rightarrow 0}{\longrightarrow} \frac{1}{\alpha^{\prime} b}$ diverged in the semi-classical limit. In the semi-classical limit of the cigar, the dilaton contribution is sub-leading to the metric.

The Virasoro primaries of the free theory are

$$
\mathcal{V}_{\alpha p_{\mathrm{L}} p_{\mathrm{R}}}(z, \bar{z})=e^{-2 \alpha \hat{r}(z, \bar{z})} e^{i p_{\mathrm{L}} \hat{\theta}_{\mathrm{L}}(z)+i p_{\mathrm{R}} \hat{\theta}_{\mathrm{R}}(\bar{z})},
$$

where $p_{\mathrm{L}}, p_{\mathrm{R}}$ are valued in the lattice

$$
p_{\mathrm{L}}=\frac{n}{\sqrt{\alpha^{\prime} k}}-\sqrt{\frac{k}{\alpha^{\prime}}} w, \quad p_{\mathrm{R}}=\frac{n}{\sqrt{\alpha^{\prime} k}}+\sqrt{\frac{k}{\alpha^{\prime}}} w, \quad n, w \in \mathbb{Z} .
$$


$n$ is the momentum number around the cylinder and $w$ is (minus) the winding number. Their conformal weights with respect to the free theory stress tensor

$$
T(z)=-\frac{1}{\alpha^{\prime}}(\partial \hat{r})^{2}-Q \partial^{2} \hat{r}-\frac{1}{\alpha^{\prime}}(\partial \hat{\theta})^{2}
$$

are

$$
h_{\alpha p_{\mathrm{L}} p_{\mathrm{R}}}=\alpha^{\prime} \alpha(Q-\alpha)+\alpha^{\prime} \frac{p_{\mathrm{L}}^{2}}{4}, \quad \bar{h}_{\alpha p_{\mathrm{L}} p_{\mathrm{R}}}=\alpha^{\prime} \alpha(Q-\alpha)+\alpha^{\prime} \frac{p_{\mathrm{R}}^{2}}{4} .
$$

The central charge of the Virasoro algebra is

$$
c_{\mathrm{LD} \times S^{1}}=2+6 \alpha^{\prime} Q^{2} .
$$

The large $\hat{r}$ expansion of the coset primary $\mathcal{O}_{j n w}(z, \bar{z})$ is ${ }^{14,15}[3]$

$$
\mathcal{O}_{j n w} \stackrel{\hat{r} \rightarrow \infty}{\longrightarrow}\left(e^{-2 Q(1-j) \hat{r}}+R(j, n, w) e^{-2 Q j \hat{r}}\right) e^{i p_{\mathrm{L}} \hat{\theta}_{\mathrm{L}}+i p_{\mathrm{R}} \hat{\theta}_{\mathrm{R}}},
$$

where $R(j, n, w)$ is the reflection coefficient $[6,9,11]$ :

$$
\begin{aligned}
R(j, n, w)= & (\nu(k))^{2 j-1} \frac{\Gamma\left(1-\frac{2 j-1}{k-2}\right)}{\Gamma\left(1+\frac{2 j-1}{k-2}\right)} \\
& \times 4^{2 j-1} \frac{\Gamma(1-2 j)}{\Gamma(2 j-1)} \frac{\Gamma\left(j+\frac{|n|-k w}{2}\right) \Gamma\left(j+\frac{|n|+k w}{2}\right)}{\Gamma\left(1-j+\frac{|n|-k w}{2}\right) \Gamma\left(1-j+\frac{|n|+k w}{2}\right)} .
\end{aligned}
$$

It satisfies $R(1-j, n, w) R(j, n, w)=1 . \nu(k)$ is a $j$-independent function, analogous to the prefactor $\pi \mu \Gamma\left(b_{\mathrm{L}}^{2}\right) / \Gamma\left(1-b_{\mathrm{L}}^{2}\right)$ appearing in the Liouville reflection coefficient eq. (2.24). We will set it to one in what follows.

As recalled in the previous section, the zero-mode wavefunction for the state prepared by inserting $\mathcal{O}_{j n w}$ in the far past on the cylinder is obtained after rescaling by $e^{-\Phi}=$ $e^{-\Phi_{0}} \cosh (r)$. For large $r$, the radial wavefunction is then

$$
\Psi_{j n w}\left(\hat{r}_{0}\right) \stackrel{\hat{r}_{0} \rightarrow \infty}{\longrightarrow} \frac{1}{2} e^{-\Phi_{0}}\left(e^{2 Q\left(j-\frac{1}{2}\right) \hat{r}_{0}}+R(j, n, w) e^{-2 Q\left(j-\frac{1}{2}\right) \hat{r}_{0}}\right) .
$$

With $j \in \frac{1}{2}+i \mathbb{R}$, neither exponential dominates the other, the asymptotic radial wavefunction is oscillatory, and one obtains a delta-function normalizable state. The asymptotic operator is identified with the linear-dilaton primary $e^{-2 \alpha \hat{r}}$ with $\alpha=Q(1-j)$ plus its reflection $e^{-2(Q-\alpha) \hat{r}}$, together with a compact boson primary of momentum $n$ and winding $-w$.

\footnotetext{
${ }^{14}$ The finite $k$ corrections to the background eq. (3.2) imply $Q=\frac{1}{\sqrt{\alpha^{\prime}(k-2)}}$. In the remainder of this subsection, we use this value of $Q$ so that the formulas for the asymptotic operators and wavefunctions are valid at finite $k$. Note that with the corrected value of $Q, c_{\mathrm{LD} \times S^{1}}$ reproduces the exact central charge eq. (3.1). Likewise the conformal weights eq. (3.16) reproduce the exact weights eq. (3.10) with the dictionary discussed below.

${ }^{15}$ This choice of operator normalization does not produce a canonically normalized two-point function. Rather, the two-point function of $\mathcal{O}_{j n w}$ and $\mathcal{O}_{j,-n,-w}$ is proportional to $R(j, n, w)$. Note that $R$ given in eq. (3.19) is appropriately even in both $n$ and $w$.
} 
For real $j$, on the other hand, the reflected term is exponentially sub-dominant for $j>\frac{1}{2}$. Then, generically, the wavefunction diverges exponentially at weak-coupling, and the associated state is non-normalizable.

There is an important exception, however, when $R(j, n, w)$ is singular, and this is the manifestation of the bound states. Indeed, on the real branch with $j=j_{N}$ given by eq. (3.9b), one of the two Gamma functions $\Gamma\left(j+\frac{|n| \pm k w}{2}\right)$ in eq. (3.19) has a simple pole, depending on the sign of $w$. For $w>0$ one has

$$
j_{N}+\frac{|n|-k w}{2}=-N
$$

and therefore

$$
\Gamma\left(j+\frac{|n|-k w}{2}\right) \stackrel{j \rightarrow j_{N}}{\longrightarrow} \frac{1}{j-j_{N}} \frac{(-)^{N}}{N !}+\mathcal{O}(1)
$$

$\Gamma\left(j_{N}+\frac{|n|+k w}{2}\right)$ is similarly singular for $w<0$. The remaining Gamma functions have additional singularities, but they are not associated to bound states [24].

Thus, for $j=j_{N}$, it is the reflected component $R\left(j_{N}, n, w\right) e^{-2 Q j_{N} \hat{r}}$ that dominates in the asymptotic region. One obtains a discrete set of operators $\tilde{\mathcal{O}}_{j_{N} n w}$ defined as the residue of $\mathcal{O}_{j n w}$ as $j \rightarrow j_{N}$ [24]. The zero-mode radial wavefunction decays in the weak-coupling region, corresponding to a normalizable bound state with wavefunction proportional to

$$
\Psi_{j_{N} n w}\left(\hat{r}_{0}\right) \underset{\hat{r}_{0} \rightarrow \infty}{\stackrel{\propto}{\longrightarrow}} e^{-2 Q\left(j_{N}-\frac{1}{2}\right) \hat{r}_{0}} .
$$

The simplest pair of bound states have $n=0, w= \pm 1$, and $N=1$, such that $j_{N}=\frac{k}{2}-1$. The asymptotic form of these operators is

$$
\frac{1}{R} \mathcal{O}_{j=\frac{k}{2}-1, n=0, w= \pm 1} \stackrel{\hat{r} \rightarrow \infty}{\longrightarrow} e^{-\sqrt{\frac{k-2}{\alpha^{\prime}}} \hat{r}} e^{\mp i \sqrt{\frac{k}{\alpha^{\prime}}}\left(\hat{\theta}_{\mathrm{L}}-\hat{\theta}_{\mathrm{R}}\right)}
$$

The sum of these two winding operators is called the sine-Liouville operator [27]. It defines a normalizable, marginal bound state for $k>3$. For $k<3, j=\frac{k}{2}-1$ falls below the lower-bound $j>\frac{1}{2}$. Then the zero-mode wavefunction eq. (3.23) diverges at large $\hat{r}$ and the state becomes non-normalizable.

\subsection{Asymptotic conditions in the cigar}

As in the linear-dilaton theories discussed in the section 2, the dilaton's coupling to curvature produces source terms in the equations of motion on $S^{2}$ with a singular metric. Choosing the cylinder metric, the radial equation of motion eq. (3.4a) in the absence of any insertions may be written

$$
\partial \bar{\partial} r-\tanh (r) \operatorname{sech}^{2}(r) \partial \theta \bar{\partial} \theta=-\frac{\pi}{k}\left(\delta(z, \bar{z})+\delta\left(z-z_{\infty}, \bar{z}-\bar{z}_{\infty}\right)\right) \tanh (r) .
$$

At large $r$, we recover the free linear-dilaton equation of motion eq. (2.17),

$$
\partial \bar{\partial} \hat{r}=-\pi \alpha^{\prime} Q\left(\delta(z, \bar{z})+\delta\left(z-z_{\infty}, \bar{z}-\bar{z}_{\infty}\right)\right),
$$


with Green functions

$$
\hat{r}(\rho, \phi) \stackrel{\rho \rightarrow \pm \infty}{\longrightarrow} \pm \alpha^{\prime} Q \rho+\mathcal{O}(1)
$$

where $z=e^{\rho+i \phi}$. These free Green functions are self-consistent solutions of the full cigar equations of motion in the neighborhood of the source terms because the ends of the cylinder are mapped to the asymptotic region, where the corrections to the linear-dilaton $\times S^{1}$ equations of motion are exponentially sub-leading. Then just as before we may write a regulated action for the cigar on a cylinder worldsheet with linear boundary terms at its ends:

$$
\begin{aligned}
S= & \frac{k}{4 \pi} \int_{-L}^{L} \mathrm{~d} \rho \int_{0}^{2 \pi} \mathrm{d} \phi\left(\left(\partial_{\rho} r\right)^{2}+\left(\partial_{\phi} r\right)^{2}+\tanh ^{2}(r)\left(\left(\partial_{\rho} \theta\right)^{2}+\left(\partial_{\phi} \theta\right)^{2}\right)\right) \\
& -\int_{0}^{2 \pi} \frac{\mathrm{d} \phi}{2 \pi}\left(\left.r\right|_{\rho=L}+\left.r\right|_{\rho=-L}\right)+\frac{L}{k} .
\end{aligned}
$$

Next consider the equations of motion in the neighborhood of an insertion $\mathcal{O}_{j n w}\left(z^{\prime}, \bar{z}^{\prime}\right)$ away from the curvature singularities. Suppose $\operatorname{Re}(j)>\frac{1}{2}$, with $R(j, n, w)$ regular, such that the operator is dominated by $\mathcal{V}_{Q(1-j) p_{\mathrm{L}} p_{\mathrm{R}}}\left(z^{\prime}, \bar{z}^{\prime}\right)$ at large $\hat{r}$. The Green functions of the asymptotic linear-dilaton $\times S^{1}$ background in the presence of this source are

$$
\begin{aligned}
& \hat{r}(z, \bar{z}) \stackrel{\left|z-z^{\prime}\right| \rightarrow 0}{\longrightarrow} 2 \alpha^{\prime} Q(1-j) \log \left|z-z^{\prime}\right|+\mathcal{O}(1) \\
& \hat{\theta}(z, \bar{z}) \stackrel{\left|z-z^{\prime}\right| \rightarrow 0}{\longrightarrow}-\frac{i}{2} \sqrt{\frac{\alpha^{\prime}}{k}}\left(2 n \log \left|z-z^{\prime}\right|-k w \log \frac{z-z^{\prime}}{\bar{z}-\bar{z}^{\prime}}\right)+\mathcal{O}(1)
\end{aligned}
$$

Suppose furthermore that $\operatorname{Re}(j)>1$. Then the neighborhood of the insertion is mapped to $\hat{r} \rightarrow \infty$, and once again one obtains a self-consistent solution of the cigar equations of motion.

By contrast, if $j=j_{N}$, such that $R(j, n, w)$ is singular and the operator approaches $\mathcal{V}_{Q j, p_{\mathrm{L}} p_{\mathrm{R}}}$ at large $\hat{r}$, then the free radial Green function in the neighborhood of the (appropriately normalized) operator insertion is

$$
\hat{r}(z, \bar{z}) \stackrel{\left|z-z^{\prime}\right| \rightarrow 0}{\longrightarrow} 2 \alpha^{\prime} Q j \log \left|z-z^{\prime}\right|+\mathcal{O}(1)
$$

In this case, even if $\hat{r}$ begins in the asymptotic region, as one approaches the insertion point on the worldsheet $\hat{r}$ is mapped out of the free-field region, and one no longer has a self-consistent solution.

Away from these discrete values, however, the appropriate asymptotic conditions are obtained from the free-field Green functions, and correlation functions with bound state insertions may be obtained by computing the functional integral for generic $j$ and then taking the residue of the result as $j \rightarrow j_{N}$. It is nevertheless interesting to identify an asymptotic condition that describes a bound state insertion directly, rather than as the residue of an ordinary insertion. We return to this problem in section 4.3. 
If a generic operator is inserted in the far past on the cylinder, then the asymptotic conditions including the effect of the background-charge are

$$
\begin{aligned}
& \hat{r}(\rho, \phi) \stackrel{\rho \rightarrow-\infty}{\longrightarrow}-2 \alpha^{\prime} Q\left(j-\frac{1}{2}\right) \rho+\mathcal{O}(1) \\
& \hat{\theta}(\rho, \phi) \stackrel{\rho \rightarrow-\infty}{\longrightarrow}-i \sqrt{\frac{\alpha^{\prime}}{k}}(n \rho-i k w \phi)+\mathcal{O}(1) .
\end{aligned}
$$

For $\operatorname{Re}(j)>\frac{1}{2}$, the solution is consistent. A complex branch operator may be similarly described by perturbing $j \rightarrow j+\varepsilon$ by a small positive regulator.

The $\rho$ dependence of the asymptotic conditions may be enforced as before by linear boundary terms. The $\phi$ dependence of $\hat{\theta}$, on the other hand, may be implemented using Lagrange multipliers $\sigma_{ \pm}$. For example, the regulated action for the 2-point function of $\mathcal{O}_{j n w}$ and $\mathcal{O}_{j,-n,-w}$ is given by

$$
\begin{aligned}
S_{j n w}= & \frac{k}{4 \pi} \int_{-L}^{L} \mathrm{~d} \rho \int_{0}^{2 \pi} \mathrm{d} \phi\left(\left(\partial_{\rho} r\right)^{2}+\left(\partial_{\phi} r\right)^{2}+\tanh ^{2}(r)\left(\left(\partial_{\rho} \theta\right)^{2}+\left(\partial_{\phi} \theta\right)^{2}\right)\right) \\
& +2\left(\frac{1}{2}-j\right) \int_{0}^{2 \pi} \frac{\mathrm{d} \phi}{2 \pi}\left(\left.r\right|_{\rho=L}+\left.r\right|_{\rho=-L}\right)+i n \int_{0}^{2 \pi} \frac{\mathrm{d} \phi}{2 \pi}\left(\left.\theta\right|_{\rho=L}-\left.\theta\right|_{\rho=-L}\right) \\
& +k \int_{0}^{2 \pi} \frac{\mathrm{d} \phi}{2 \pi}\left(\sigma_{+}\left(\left.\partial_{\phi} \theta\right|_{\rho=L}+w\right)+\sigma_{-}\left(\left.\partial_{\phi} \theta\right|_{\rho=-L}+w\right)\right) \\
& +4 \frac{L}{k}\left(j-\frac{1}{2}\right)^{2}-k w^{2} L-\frac{L}{k} n^{2} .
\end{aligned}
$$

Note that the imaginary boundary term for the momentum mode of $\theta$ ensures invariance of $e^{-S_{\text {jnw }}}$ under $\theta \sim \theta+2 \pi$, where $n \in \mathbb{Z}$.

The boundary equations of motion obtained by varying $r$ and $\sigma_{ \pm}$are

$$
\begin{aligned}
& \left.\partial_{\rho} r\right|_{\rho= \pm L}= \pm \frac{2}{k}\left(j-\frac{1}{2}\right) \\
& \left.\partial_{\phi} \theta\right|_{\rho= \pm L}=-w,
\end{aligned}
$$

while the variation of $\theta$ gives

$$
\pm\left.\partial_{\phi} \sigma_{ \pm}\right|_{\rho= \pm L}=\frac{i n}{k}+\left.\tanh ^{2}(r) \partial_{\rho} \theta\right|_{\rho= \pm L}
$$

In the large $L$ limit, eq. (3.33a) implies the asymptotic condition

$$
r \stackrel{\rho \rightarrow \pm \infty}{\longrightarrow} \pm \frac{2}{k}\left(j-\frac{1}{2}\right) \rho
$$

as in eq. (3.31a). Eq. (3.33b) requires that $\theta \rightarrow-w \phi+\theta_{0}(\rho)$, where $\theta_{0}(\rho)$ is the zero-mode in the Fourier expansion of $\theta(\rho, \phi)$ around the $\phi$ circle. This zero-mode is meanwhile fixed 
by the integral of eq. (3.34),

$$
\left.\int_{0}^{2 \pi} \mathrm{d} \phi \tanh ^{2}(r) \partial_{\rho} \theta\right|_{\rho= \pm \infty}=-\frac{2 \pi i n}{k} .
$$

Note that $\left.\tanh ^{2}(r)\right|_{\rho= \pm \infty}$ goes to one in large $L$ limit. The asymptotic condition on $\theta$ is then

$$
\theta \stackrel{\rho \rightarrow \pm \infty}{\longrightarrow}-i \frac{n}{k} \rho-w \phi
$$

reproducing eq. (3.31b).

In other words, eq. (3.33b) is a Dirichlet condition that requires the non-zero-modes of $\theta$ to vanish at the boundaries, while the integral of eq. (3.34) is a Neumann condition on the zero-mode. One solves the bulk equations of motion with these boundary conditions, together with the Neumann condition eq. (3.33a) on $r$. The Lagrange multipliers are then determined by eq. (3.34) up to a zero-mode, which we discard.

The functional integral weighted by $e^{-S_{j n w}}$ computes the reflection coefficient $R(j, n, w)$ in the $L \rightarrow \infty$ limit. In the next section, we compute the saddle-point expansion of this integral, focusing on the pure-winding sector, and show that there exists a set of saddles that reproduces the semi-classical limit of the exact reflection coefficient.

\section{Semi-classical limit}

In this section we compute the semi-classical limit of the cigar reflection coefficient by a saddle-point expansion, and compare to the large $k$ limit of the exact reflection coefficient eq. (3.19). As in the analogous calculation for Liouville [1], doing so requires summing over complex saddles, even for real branch operators. We will also find that saddles which hit the black hole singularity contribute to the saddle-point expansion with finite action, and are important for recovering the real branch bound states.

\subsection{The reflection coefficient in the large $k$ limit}

As reviewed in section 3.2, the exact reflection coefficient $R(j, n, w)$ of the $\mathrm{SL}(2, \mathbb{R})_{k} / \mathrm{U}(1)$ CFT is known thanks to work on the $\mathrm{SL}(2, \mathbb{R})_{k}$ WZW model (and its Euclidean continuation $\left.\mathrm{SL}(2, \mathbb{C})_{k} / \mathrm{SU}(2)\right)$, and its relation to $\mathrm{SL}(2, \mathbb{R})_{k} / \mathrm{U}(1)$ via the coset construction $[6,9,11]$. $R(j, n, w)$ defines the normalization of the 2-point function of the coset primaries $\mathcal{O}_{j n w}$ and $\mathcal{O}_{j,-n,-w}$, with the operator normalization chosen in eq. (3.18). Physically, it is the amplitude for a string sent from the weak-coupling region to reflect off the tip of the cigar.

In this section we will primarily focus on the pure-winding sector $n=0$, which includes the simplest bound states. In section 4.4 we discuss the closely analogous calculation for the pure-momentum states $w=0$, whose continuation to target space Lorentzian signature describe scattering states in the black hole background. For the winding states, eq. (3.19) simplifies to

$$
R(j, w)=4^{2 j-1} \frac{k-2}{\gamma\left(\frac{2 j-1}{k-2}\right)} \frac{1}{\gamma(2 j)} \gamma\left(j+\frac{1}{2} k w\right) \gamma\left(j-\frac{1}{2} k w\right),
$$

where $\gamma(z) \equiv \Gamma(z) / \Gamma(1-z)$. 
The cigar-sigma model description of the CFT is weakly-coupled for large $k$, and our goal in this section is to compute $R(j, w)$ by a saddle-point expansion in the $k \rightarrow \infty$ limit. In order to compare with the same limit of the exact result, let us first determine the large $k$ asymptotics of eq. (4.1). To do so, we must first decide how $j$ scales with $k$. We will restrict our attention to "heavy" operators, whose insertions contribute at the same order in $k$ as the leading terms in the action. We therefore define

$$
j=\frac{k \eta}{2},
$$

with $\eta=\mathcal{O}\left(k^{0}\right)$. Imposing $\operatorname{Re}(j)>\frac{1}{2}$ requires $\operatorname{Re}(\eta)>\frac{1}{k}$, which relaxes to $\operatorname{Re}(\eta)>0$ in the large $k$ limit.

The asymptotic behavior of the Gamma function for large complex values of its argument depends on the direction in the complex plane in which the limit is taken. To $e^{\mathcal{O}\left(z^{-1}\right)}$, it is given by $[1,28,29]$

$$
\Gamma(z) \stackrel{|z| \rightarrow \infty}{\longrightarrow} \begin{cases}e^{\left(z-\frac{1}{2}\right) \log (z)-z+\frac{1}{2} \log (2 \pi)+\mathcal{O}\left(z^{-1}\right)} & \operatorname{Re}(z)>0 \\ \csc (\pi z) e^{\left(z-\frac{1}{2}\right) \log (-z)-z+\frac{1}{2} \log \left(\frac{\pi}{2}\right)+\mathcal{O}\left(z^{-1}\right)} & \operatorname{Re}(z)<0 .\end{cases}
$$

The first line is the usual Stirling approximation, and the second follows from the first in combination with the identity $\Gamma(z) \Gamma(-z)=-\frac{\pi}{z} \csc (\pi z)$. The asymptotics of $\gamma(z)$ are then

$$
\gamma(z) \stackrel{|z| \rightarrow \infty}{\longrightarrow} \begin{cases}\frac{2 \sin (\pi z)}{z} e^{2 z(\log (z)-1)+\mathcal{O}\left(z^{-1}\right)} & \operatorname{Re}(z)>0 \\ -\frac{\csc (\pi z)}{2 z} e^{2 z(\log (-z)-1)+\mathcal{O}\left(z^{-1}\right)} & \operatorname{Re}(z)<0 .\end{cases}
$$

Assuming without loss of generality that $w>0$, we obtain

$$
\begin{aligned}
R(\eta, w) \stackrel{k \rightarrow \infty}{\longrightarrow} & \eta^{-2 k \eta}(w+\eta)^{k(w+\eta)} \csc (\pi k \eta) \sin \left(\frac{\pi}{2} k(w+\eta)\right) \\
& \times \begin{cases}\frac{1}{2}(w-\eta)^{k(\eta-w)} \csc \left(\frac{\pi}{2} k(w-\eta)\right) & 0<\operatorname{Re}(\eta)<w \\
2(\eta-w)^{k(\eta-w)} \sin \left(\frac{\pi}{2} k(\eta-w)\right) & \operatorname{Re}(\eta)>w\end{cases} \\
& \times \frac{\eta}{\left(\eta^{2}-w^{2}\right) \gamma(\eta)} .
\end{aligned}
$$

We have kept terms to order $k^{0}$ in the exponent. Note that the bound states now correspond to the poles of $\csc \left(\frac{\pi}{2} k(w-\eta)\right)$ at $\eta_{N}=w-\frac{2 N}{k}$. When we compute the saddle-point expansion, we expect the contribution $\sum e^{-S+\mathcal{O}\left(k^{0}\right)}$ from the order $k$ action evaluated on its saddles to reproduce the first two lines. The last line is order $e^{k^{0}}$, which we expect to arise from the fluctuation determinant, as well as the order $k^{0}$ corrections to the on-shell action. ${ }^{16}$

\footnotetext{
${ }^{16}$ It has been suggested that the cigar sigma-model is supplemented even at large $k$ by a potential that modifies the background in the neighborhood of the tip [30-32]. It would be interesting to see if the third line of eq. (4.5), in particular the factor of $\gamma(\eta)$ which originated in the factor of $\gamma\left(\frac{2 j-1}{k-2}\right)$ in eq. (4.1), is correctly reproduced by the 1-loop calculation in the pure cigar background. We have not attempted to compute this determinant, however.
} 


\subsection{Saddle-point expansion}

We now turn to the calculation of the semi-classical reflection coefficient by a saddle-point expansion of the functional integral

$$
R(\eta, w)=\int_{\mathcal{C}(\eta)} \operatorname{Dr} D \theta D \sigma_{ \pm} e^{-k \tilde{S}_{j w}},
$$

with action $k \tilde{S}_{j w} \equiv S_{j, 0, w}$ (cf. eq. (3.32)):

$$
\begin{aligned}
\tilde{S}_{j w}= & \frac{1}{4 \pi} \int_{-L}^{L} \mathrm{~d} \rho \int_{0}^{2 \pi} \mathrm{d} \phi\left(\left(\partial_{\rho} r\right)^{2}+\left(\partial_{\phi} r\right)^{2}+\tanh ^{2}(r)\left(\left(\partial_{\rho} \theta\right)^{2}+\left(\partial_{\phi} \theta\right)^{2}\right)\right) \\
& -\left(\eta-\frac{1}{k}\right) \int_{0}^{2 \pi} \frac{\mathrm{d} \phi}{2 \pi}\left(\left.r\right|_{\rho=L}+\left.r\right|_{\rho=-L}\right)+L(\eta-1 / k)^{2}-w^{2} L \\
& +\int_{0}^{2 \pi} \frac{\mathrm{d} \phi}{2 \pi}\left(\sigma_{+}\left(\left.\partial_{\phi} \theta\right|_{\rho=L}+w\right)+\sigma_{-}\left(\left.\partial_{\phi} \theta\right|_{\rho=-L}+w\right)\right) .
\end{aligned}
$$

As discussed in section 3.3, the maps $r$ and $\theta \sim \theta+2 \pi$ are defined on a worldsheet cylinder $[-L, L] \times S^{1}$ whose length $2 L$ is taken to infinity. In this limit, the boundary terms insert the operators $\mathcal{O}_{j=\frac{k \eta}{2}, n=0, \pm w}$, as well as the background-charge contributions, on opposite ends of the cylinder, and the functional integral computes the reflection coefficient. In the $k \rightarrow \infty$ limit, we would like to evaluate this integral by a saddle-point expansion

$$
R(\eta, w) \stackrel{k \rightarrow \infty}{\longrightarrow} \sum_{r_{i}, \theta_{i}} e^{-k \tilde{S}_{j w}+\mathcal{O}\left(k^{0}\right)},
$$

where $\left\{r_{i}, \theta_{i}\right\}$ are a subset of solutions of the equations of motion

$$
\begin{aligned}
& \left(\partial_{\rho}^{2}+\partial_{\phi}^{2}\right) r-\tanh (r) \operatorname{sech}^{2}(r)\left(\left(\partial_{\rho} \theta\right)^{2}+\left(\partial_{\phi} \theta\right)^{2}\right)=0 \\
& \left(\partial_{\rho}^{2}+\partial_{\phi}^{2}\right) \theta+2 \operatorname{sech}(r) \operatorname{csch}(r)\left(\partial_{\rho} r \partial_{\rho} \theta+\partial_{\phi} r \partial_{\phi} \theta\right)=0
\end{aligned}
$$

in the bulk and

$$
\begin{aligned}
\left.\partial_{\rho} r\right|_{\rho= \pm L} & = \pm \eta \\
\left.\partial_{\phi} \theta\right|_{\rho= \pm L} & =-w \\
\left.\int_{0}^{2 \pi} \mathrm{d} \phi \tanh ^{2}(r) \partial_{\rho} \theta\right|_{\rho= \pm L} & =0
\end{aligned}
$$

on the boundaries. ${ }^{17}$ We have discarded here the contributions from the $\mathcal{O}\left(k^{-1}\right)$ terms in $\tilde{S}_{j w}$, which contribute to the order one corrections to the saddle-point expansion.

\footnotetext{
${ }^{17}$ As explained in section 3.3, the Lagrange multipliers are then determined on-shell from $\partial_{\phi} \sigma_{ \pm}=$ $\pm\left.\tanh ^{2}(r) \partial_{\rho} \theta\right|_{\rho= \pm L}$ up to a $\phi$ zero-mode, which we discard. Evaluated on $\theta=-w \phi$, one obtains $\partial_{\phi} \sigma_{ \pm}=0$, and we then gauge-fix $\sigma_{ \pm}=0$.
} 
In the limit $L \rightarrow \infty$, the boundary equations of motion for $r$ impose the asymptotic conditions

$$
r \stackrel{\rho \rightarrow \pm \infty}{\longrightarrow} \pm \eta \rho+a_{ \pm}
$$

which sends $r$ to the weak-coupling region since $\operatorname{Re}(\eta)>0$. The integration constants $a_{ \pm}$ are sub-leading, but control the asymptotic separation $r(\infty)-r(-\infty)=a_{+}-a_{-}$.

Applying eq. (4.11) in the boundary $\theta$ equations of motion allows us to discard the factor of $\tanh ^{2}(r)$ in the large $L$ limit. Then these equations imply the asymptotic condition

$$
\theta \stackrel{\rho \rightarrow \pm \infty}{\longrightarrow}-w \phi
$$

which demands that $\theta$ have winding $-w$ around the ends of the cylinder. The simplest solution sets $\theta=-w \phi$ everywhere, on which the equations of motion reduce to

$$
\begin{aligned}
\partial_{\rho}^{2} r-w^{2} \tanh (r) \operatorname{sech}^{2}(r) & =0 \\
\partial_{\phi} r & =0 \\
\left.\partial_{\rho} r\right|_{\rho= \pm L} & = \pm \eta .
\end{aligned}
$$

Thus, in this pure-winding sector the theory reduces to a quantum mechanics problem for $r(\rho)$ with action

$$
\tilde{S}[r]=\int_{-L}^{L} \mathrm{~d} \rho\left(\frac{1}{2}\left(\frac{\mathrm{d} r}{\mathrm{~d} \rho}\right)^{2}+V(r)\right)-\eta(r(L)+r(-L))+\eta^{2} L,
$$

where

$$
V(r)=-\frac{1}{2} w^{2} \operatorname{sech}^{2}(r)
$$

The bulk equation of motion eq. (4.13a) may be written

$$
\frac{\mathrm{d}^{2} r}{\mathrm{~d} \rho^{2}}=V^{\prime}(r)
$$

describing the mechanics of a particle in the inverted potential $-V(r)$, pictured in figure 2 . The quantum mechanics of a particle in this potential can in fact be solved exactly, as we review in appendix A. There we show that the semi-classical limit of the exact reflection coefficient for the quantum mechanics, eq. (A.35), reproduces that of the CFT, eq. (4.5), at order $e^{k}$. We conclude that the saddles with $\theta(\phi)=-w \phi$ and $r=r(\rho)$ are sufficient for reproducing the saddle-point expansion of the coset reflection coefficient, and we do not need to consider more complicated solutions $\theta(\rho, \phi)$ and $r(\rho, \phi)$.

Since we allow for complex values of $\eta$, clearly the saddles of the functional integral will in general be complex. In fact, even for real values of $\eta$ we will see that one must sum over complex saddles. The necessity of complexification is familiar from applications of the saddle-point method to asymptotic expansions of ordinary integrals over real variables, where the original real integration contour is typically deformed into a homotopically equivalent sum of steepest-descent contours passing through complex critical points. 


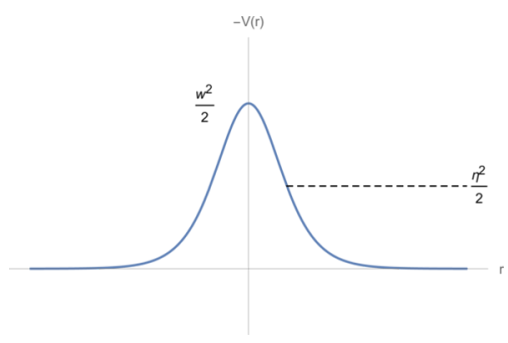

Figure 2. Inverted potential. Restricted to a pure-winding solution $\theta=-w \phi$, the cigar equations of motion describe the mechanics of a particle moving in the inverted potential shown. For $\eta$ real and less than $w$, there is a real solution that describes a particle that comes in from $r \rightarrow \infty$, rolls partway up the potential hill until it stops at the turning point, and then rolls back out to infinity. The cigar geometry is defined for $r \geq 0$, but to compute the saddle-point expansion of the functional integral we will continue $r$ to the complex plane. Here we draw the potential for the real $r$ slice.

The asymptotic expansion of the Gamma function in eq. (4.3) may itself be understood as a finite-dimensional example of the saddle-point expansion, since $\Gamma(z)$ may be defined by the integral

$$
\Gamma(z)=\int_{\mathcal{C}(z)} \mathrm{d} X e^{-\left(-z X+e^{X}\right)} .
$$

We refer the reader to appendix $\mathrm{C}$ of [1] for a self-contained review, because the problem for the functional integral is in many ways analogous. Briefly, the saddle-points of the "action" $S[X]=-z X+e^{X}$ are given by $X_{N}=\log (z)+2 \pi i N$, with $N \in \mathbb{Z}$. The contour $\mathcal{C}(z)$ is given by the real axis for $\operatorname{Re}(z)>0$, though it must be deformed for $\operatorname{Re}(z)<0$ to preserve convergence of the integral. For $\operatorname{Re}(z)>0, \mathcal{C}(z)$ is homotopic to the steepest-descent contour $\mathcal{C}_{0}$ through $X_{0}$, and one recovers the Stirling formula $e^{-S\left[X_{0}\right]}=e^{z \log (z)-z}$.

Along the imaginary $z$-axis, however, one encounters what is known as a Stokes wall. There the steepest-descent contour of any saddle-point, which otherwise varies smoothly with $z$, collides with a neighboring saddle-point. As a result, for values of $z$ just to either side of the imaginary axis, the steepest-descent contour jumps discontinuously. The integration contour $\mathcal{C}(z)$ itself varies smoothly with $z$, but its expansion in steepest-descent contours changes abruptly upon crossing the Stokes wall, and therefore its asymptotic expansion changes as well. For $\operatorname{Re}(z)<0$ and $\operatorname{Im}(z)>0, \mathcal{C}(z)$ is instead homotopic to the sum of steepest-descent contours $\sum_{N=0}^{\infty} \mathcal{C}_{N}$ passing through the saddles $X_{N}$. Then the saddle-point expansion yields

$$
\begin{aligned}
\sum_{N=0}^{\infty} e^{-S\left[X_{N}\right]} & =e^{-S\left[X_{0}\right]} \sum_{N=0}^{\infty} e^{2 \pi i z N} \\
& =\csc (\pi z) e^{z \log (-z)-z+\mathcal{O}\left(z^{0}\right)}
\end{aligned}
$$

as in eq. (4.3). For $\operatorname{Im}(z)<0$, the relevant contours are instead $\sum_{N=0}^{\infty} \mathcal{C}_{-N}$, as required for the geometric series to converge.

Thus, the poles of the Gamma function on the negative real axis may be understood as the divergence of the geometric series $\sum_{N=0}^{\infty} e^{2 \pi i z N}$ that results from summing over a 
family of saddles related by complex shifts $X \rightarrow X+2 \pi i$. We will see that the poles of $\csc (\pi k \eta)$ in eq. (4.5) are of similar origin. The poles of $\csc \left(\frac{\pi}{2} k(w-\eta)\right)$, meanwhile, will be attributed to summing over a family of singular saddles with finite action. In section 5 , we will also see that the poles of the prefactor $\gamma\left(\frac{2 j-1}{k-2}\right)$ in eq. (4.1) are due to a complex shift symmetry of the dual sine-Liouville description of the coset CFT in the $k \rightarrow 2$ limit.

As in the finite-dimensional problem, the saddle-point expansion of a functional integral is performed by deforming the integration contour into a sum of complex cycles $[1,13]$. Unlike for a finite-dimensional integral, however, for a functional integral it is in general very challenging to determine the set of steepest-descent cycles that are homotopic to the original contour. In other words, it is a hard problem to derive from first principles which complex saddles one should sum over in computing the saddle-point expansion, especially since the necessary set of saddles can jump upon crossing Stokes walls in the parameter space. Since for the problem at hand we know the exact answer and its semi-classical limit eq. (4.5), the approach we take here is to show that there exists a consistent set of solutions for which the saddle-point expansion reproduces the known answer.

In light of the abrupt change in the semi-classical limit of $R(\eta, w)$ across the line $\operatorname{Re}(\eta)=w$, we anticipate that a Stokes wall in the $\eta$-plane is found there. This is not unreasonable, since for $\eta$ real and less than $w$ there exists a real solution of the equations of motion describing a particle that comes in from $r \rightarrow \infty$, rolls partway up the potential until it stops at the turning point, and then rolls back out to infinity. For $\eta$ greater than $w$, on the other hand, the particle rolls over the potential and continues to $r \rightarrow-\infty$ in the continued field space. At the crossover point, the particle has just enough energy to (asymptotically) reach the top of the hill.

Thus, in computing the saddle-point expansion we expect that we will need to address the domains for $0<\operatorname{Re}(\eta)<w$ and $\operatorname{Re}(\eta)>w$ separately, and that we will find a different set of contributing saddles in each.

Finally, we have not yet specified the contour $\mathcal{C}(\eta)$ in field space along which the functional integral eq. (4.6) is to be performed. In the example of the Gamma function, one starts with a contour along the real axis when $z$ is a positive real number. Then one deforms it as necessary for complex values of $z$ to preserve convergence of the integral and produce an analytic function of $z$.

By contrast, even for real $\eta$ the functional integral eq. (4.6) over real fields diverges. Borrowing an argument from ${ }^{18}[1]$, consider a finite-action configuration of $\left(r, \theta, \sigma_{ \pm}\right)$. Now consider another configuration with $r \rightarrow r+a$ shifted by a large, positive real number. Then the action of the latter configuration is given by

$$
\tilde{S}_{j w}[r+a] \stackrel{a \rightarrow \infty}{\longrightarrow}-2 \eta a+\tilde{S}_{j w}[r]+\frac{1}{4 \pi} \int \mathrm{d} \rho \mathrm{d} \phi \operatorname{sech}^{2}(r)(\nabla \theta)^{2}+\mathcal{O}\left(e^{-2 a}, k^{-1}\right) .
$$

Since $\operatorname{Re}(\eta)>0$, by making $a$ arbitrarily large the action may be made arbitrarily negative. Thus, we have identified a region of real field space where $e^{-S} \rightarrow \infty$, and therefore the functional integral over real fields cannot converge.

\footnotetext{
${ }^{18}$ In [1] it is similarly shown that the functional integral over real fields for the Liouville 2-point function diverges. In [23], this divergence was dealt with by a fixed-area prescription, while in [1] it was interpreted as an indication that the functional integral must instead be defined over a complex cycle.
} 
Instead, $\mathcal{C}(\eta)$ must be chosen to be an appropriate complex cycle. By identifying the set of saddles with which the semi-classical limit of the exact result is reproduced, the appropriate contour may in principle be defined by the sum of steepest-descent contours associated to these complex saddles. Away from the Stokes walls, the steepest-descent contours themselves vary smoothly with $\eta$, as does $\mathcal{C}(\eta)$ in turn. As in the finite-dimensional case, even though the steepest-descent contours jump when $\eta$ crosses a Stokes wall, $\mathcal{C}(\eta)$ is expected to vary smoothly. Its expansion as a sum of steepest-descent contours changes across the Stokes wall, but the summed contours on either side of the wall should be equivalent up to Cauchy deformation.

\subsubsection{Complex quantum mechanics}

On the pure-winding solution $\theta=-w \phi$, the cigar action has reduced to the complex quantum mechanics in eq. (4.14). Since the original real coordinate $r \geq 0$ was valued in a half-line, the relevant complexification is the complex $r$-plane quotiented by $r \sim-r$. Note that this is a symmetry of the $\operatorname{sech}^{2}(r)$ potential.

Alternatively, one may compute the saddle-point expansion for the reflection and transmission coefficients of the quantum mechanics before the quotient, and then take their difference to obtain the reflection coefficient in the half-space. This is the approach that we will take here. Thus, we regard the action $\tilde{S}[r]$ in eq. (4.14) as a holomorphic functional of maps $r: \mathbb{R} \rightarrow \mathbb{C}$ into the complex $r$-plane and identify its critical points. ${ }^{19}$

The semi-classical limits of the reflection and transmission coefficients obtained from the exact solution of the full-space quantum mechanics are (cf. eqs. (A.26) and (A.27))

$$
\begin{aligned}
& R_{\mathrm{QM}}(\eta) \\
& \stackrel{k \rightarrow \infty}{\propto} \begin{cases}\eta^{-2 k \eta}(w+\eta)^{k(w+\eta)}(w-\eta)^{k(\eta-w)} \sin (\pi k w) \csc (\pi k \eta) \csc (\pi k(w-\eta)) & 0<\operatorname{Re}(\eta)<w \\
\eta^{-2 k \eta}(w+\eta)^{k(w+\eta)}(\eta-w)^{k(\eta-w)} \sin (\pi k w) \csc (\pi k \eta) & \operatorname{Re}(\eta)>w\end{cases}
\end{aligned}
$$

and

$$
T_{\mathrm{QM}}(\eta) \stackrel{k \rightarrow \infty}{\propto} \begin{cases}\eta^{-2 k \eta}(w+\eta)^{k(w+\eta)}(w-\eta)^{k(\eta-w)} \csc (\pi k(w-\eta)) & 0<\operatorname{Re}(\eta)<w \\ \eta^{-2 k \eta}(w+\eta)^{k(w+\eta)}(\eta-w)^{k(\eta-w)} & \operatorname{Re}(\eta)>w .\end{cases}
$$

It is shown in appendix A that the reflection coefficient of the half-space quantum mechanics, $R_{\mathrm{QM}}(\eta)-T_{\mathrm{QM}}(\eta)$, reproduces the cigar reflection coefficient at order $e^{k}$. Thus, our task is reduced to reproducing eqs. (4.21) and (4.22) by saddle-point expansions for the infinite-space quantum mechanics. The rest of this section is devoted to that calculation. In the remainder of the present sub-section, we discuss some generalities about the complex quantum mechanics and its saddles. In the following two sub-sections, we compute the saddle-point expansions of the reflection and transmission coefficients.

The bulk equation of motion eq. (4.16) describes a particle moving in an inverted potential $-V(r)$. One therefore obtains the energy conservation equation,

$$
\frac{1}{2} \dot{r}^{2}-V(r)=\frac{\eta^{2}}{2}
$$

\footnotetext{
${ }^{19}$ The transmission coefficient is computed similarly, but with $r(L)$ replaced by $-r(L)$ to fix the momentum at late times to $-\eta$ rather than $\eta$.
} 
where $\dot{r}=\frac{\mathrm{d} r}{\mathrm{~d} \rho}$. The conserved energy is indeed $\frac{\eta^{2}}{2}$, as is clear by evaluating the equation at $\rho \rightarrow \pm \infty$ and imposing the asymptotic conditions.

Observe that, as a holomorphic function on the complex $r$-plane, the potential is periodic in $\pi i$ :

$$
V(r+\pi i)=V(r) .
$$

The turning points, where $-V\left(r_{ \pm}\right)=\frac{\eta^{2}}{2}$ and therefore $\dot{r}=0$, are given by

$$
r_{ \pm}= \pm \cosh ^{-1}\left(\frac{w}{\eta}\right)
$$

as well as all shifts thereof by $\pi i \mathbb{Z}$.

There are also singular points where the potential diverges. $V(r)$ has a double-pole at $r=\frac{\pi i}{2}$

$$
V(r) \stackrel{r \rightarrow \frac{\pi i}{2}}{\longrightarrow} \frac{w^{2}}{2} \frac{1}{\left(r-\frac{\pi i}{2}\right)^{2}}+\mathcal{O}(1),
$$

and likewise at all points $\frac{\pi i}{2}+\pi i \mathbb{Z}$. We point out that $r= \pm \frac{\pi i}{2}$ coincide with the physical singularities of the Lorentzian black hole after continuing $\theta$ to Lorentzian time.

To compute the saddle-point expansion, we must evaluate the action on the solutions of the equations of motion. We will obtain these explicit solutions momentarily, but it is not actually necessary to solve the equations of motion in order to compute the on-shell action. Using the energy conservation equation, we may write eq. (4.14) as

$$
\tilde{S}[r]=\int_{-L}^{L} \mathrm{~d} \rho\left(\frac{\mathrm{d} r}{\mathrm{~d} \rho}\right)^{2}-\eta(r(L)+r(-L)) .
$$

Letting $\mathscr{C}$ denote the contour traced by the solution in the complex $r$-plane, we may write the action as a contour integral:

$$
\tilde{S}[r]=\int_{\mathscr{C}} \mathrm{d} r \sqrt{\eta^{2}+2 V(r)}-\eta(r(L)+r(-L)) .
$$

Note that the integrand $\sqrt{\eta^{2}+2 V(r)}=\dot{r}$ is the velocity function, and one should pick an appropriate branch of the square-root such that the velocity has the correct sign. The turning points $r_{ \pm}+\pi i \mathbb{Z}$ are branch points of the square-root. The double-poles of the potential, meanwhile, lead to simple-poles of the integrand of residue $\pm w$ :

$$
\pm \sqrt{\eta^{2}+2 V(r)} \stackrel{r \rightarrow \frac{\pi i}{2}}{\longrightarrow} \pm \frac{w}{r-\frac{\pi i}{2}}+\mathcal{O}(1)
$$

The explicit solutions of the bulk equation of motion may be obtained by separating and integrating the energy conservation equation. One finds

$$
r(\rho)=\sinh ^{-1}\left(\sqrt{\frac{w^{2}}{\eta^{2}}-1} \cosh \left(\eta\left(\rho+i \rho_{0}\right)\right)\right)+\pi i N_{1},
$$

where $\rho_{0}$ is a complex number and $N_{1}$ is an integer. $\rho_{0}$ is the integration constant that arises in integrating the energy conservation equation. In the limit $L \rightarrow \infty$, the real part 
of $i \rho_{0}$ is merely a reparameterization of $\rho$; we therefore take $i \rho_{0}$ to be pure imaginary. The freedom to shift any solution by $\pi i N_{1}$ arises from the periodicity of the potential. For each $N_{1}$, the continuous modulus $\rho_{0}$ parameterizes a family of solutions. The on-shell action is necessarily the same for all trajectories in such a family, unless in varying $\rho_{0}$ one encounters a singular solution. ${ }^{20}$ The on-shell action does depend on the discrete parameter $N_{1}$, however, through the boundary terms.

Since $\sinh ^{-1}(z)=\log \left(z+\sqrt{z^{2}+1}\right)$ is a multi-valued function, one has to pick a branch to define the trajectory. The $\sqrt{z^{2}+1}$ term leads to square-root branch points at $z= \pm i$, and there is a logarithmic branch point at infinity. On the principal branch, the cuts extend along the imaginary axis from $i$ to $i \infty$ and from $-i$ to $-i \infty$, though other choices are convenient depending on the values of the parameters. ${ }^{21}$

Eq. (4.30) solves the bulk equation of motion, but it remains to check if it satisfies the boundary equations. The velocity function is

$$
\dot{r}=\eta \frac{\sqrt{\frac{w^{2}}{\eta^{2}}-1} \sinh \left(\eta\left(\rho+i \rho_{0}\right)\right)}{\sqrt{\left(\frac{w^{2}}{\eta^{2}}-1\right) \cosh ^{2}\left(\eta\left(\rho+i \rho_{0}\right)\right)+1}},
$$

which indeed asymptotes to $\pm \eta$ as $|\rho| \rightarrow \infty$. The sign, however, depends on the branch of the square-root in the denominator, which coincides with the branch of the square-root in $\sinh ^{-1}(z)$. Depending on the values of the parameters, one obtains either a reflected or transmitted solution.

For example, several trajectories with $\eta$ real and less than $w, 0<\rho_{0}<\frac{\pi}{2 \eta}$, and $N_{1}=0$ are plotted in figure 3. The solid disks indicate the turning points $r_{ \pm}+\pi i \mathbb{Z}$, and the open circles indicate the singularities $\frac{\pi i}{2}+\pi i \mathbb{Z}$. The blue trajectory that hugs the real axis corresponds $^{22}$ to the real solution with $\rho_{0}=0$ for a particle that rolls up and down the potential hill, turning around at $r_{+}$. For the green trajectory nearly hitting the poles, on the other hand, $\rho_{0}$ is just below $\frac{\pi}{2 \eta}$.

Upon reaching $\rho_{0}=\frac{\pi}{2 \eta}$, the asymptotic imaginary part of the trajectory reaches $\pm \frac{\pi}{2}$, and the saddle becomes singular. The argument of the $\sinh ^{-1}$ in eq. (4.30) hits the branch points at $\pm i$ at finite $\rho$. Indeed, the inverted potential on the real slice $\operatorname{Im}(r)= \pm \frac{\pi}{2}$ is an infinite well, $-V\left(x \pm \frac{\pi i}{2}\right)=-\frac{1}{2} w^{2} \operatorname{csch}^{2}(x)$, pictured in figure 4a, and a particle kicked to the left from $x>0$ falls down the well and hits the singularity. Similarly, on the imaginary axis $r=i y$, the potential experienced by $y$ is ${ }^{23} V(i y)=-\frac{1}{2} w^{2} \sec ^{2}(y)$, which is again singular, as pictured in figure 4b. Remarkably, we will see that the singular trajectories carry finite action and must be included in the saddle-point expansion to correctly reproduce

\footnotetext{
${ }^{20}$ The divergent sum over $\rho_{0}$ is attributed to the infinite $\delta(j-j)$ factor in the 2-point function of $\mathcal{O}_{j n w}$ and $\mathcal{O}_{j,-n,-w}[1]$.

${ }^{21}$ In particular, for complex $\eta$ the argument of the $\sinh ^{-1}$ in eq. (4.30) behaves as a spiral at large $|\rho|$ since $\cosh (\eta \rho) \sim e^{\eta|\rho|}$. In that case one has to pick more complicated spiral branch cuts.

${ }^{22}$ In the figure, $\rho_{0}$ is deformed slightly away from 0 so that the incoming and outgoing segments of the trajectory do not overlap.

${ }^{23}$ Note that the potential for the imaginary part of the complex coordinate has a relative minus sign, due to the combined factors of $i$. The equation of motion is $\ddot{y}=\frac{\mathrm{d}}{\mathrm{d} y}\left(\frac{1}{2} w^{2} \sec ^{2}(y)\right)$.
} 


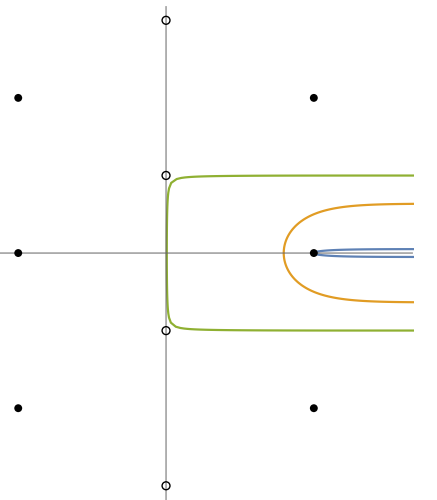

Figure 3. A family of reflected solutions. Pictured here are several solutions with $\eta$ real and less than $w, 0<\rho_{0}<\frac{\pi}{2 \eta}$, and $N_{1}=0$, obtained from eq. (4.30) with the principal branch of $\sinh ^{-1}$. The solid disks indicate the turning points and the open circles indicate the singularities. The solution hugging the real axis has $\rho_{0}$ just above zero, corresponding to the real solution that rolls up and down the same side of the inverted-potential. All trajectories related by continuously dialing $\rho_{0}$ have the same action, unless one hits a singular trajectory in the process. The green solution pictured is nearly singular, with $\rho_{0}$ just below $\frac{\pi}{2 \eta}$. At that value the trajectory will hit the poles of the potential.

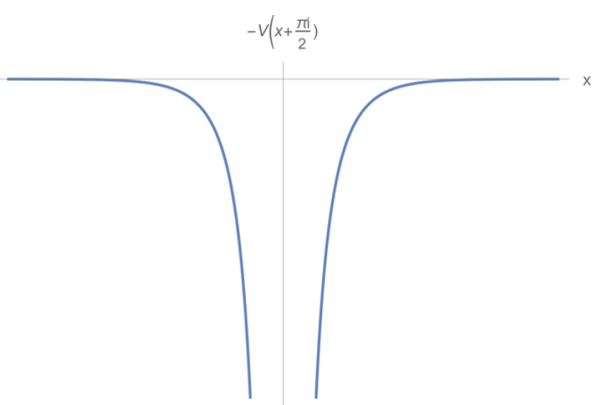

(a)

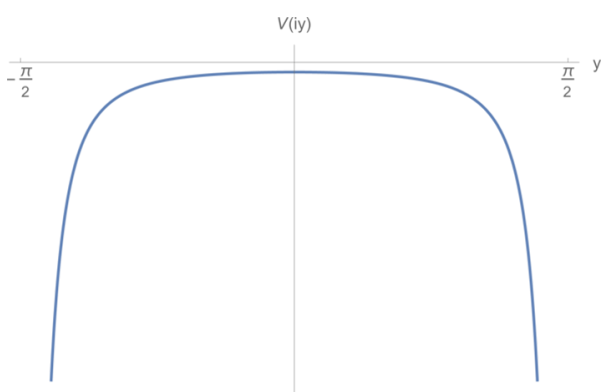

(b)

Figure 4. Singular potentials. On the slices $\operatorname{Im}(r)=\frac{\pi}{2}$ (left) and $\operatorname{Re}(r)=0$ (right), the potential experienced by the particle falls to $-\infty$ at the singular points.

the semi-classical reflection coefficient. The importance of singular saddles was discussed in closely related contexts in $[1,18]$.

\subsubsection{Reflection coefficient on the complex $r$-plane}

We now consider the saddle-point expansion of the reflection coefficient for the complex quantum mechanics on the full $r$-plane. Let us begin by computing the action of the real saddle that exists for $0<\eta<w$. We need to evaluate eq. (4.28) for the contour around the positive real axis in figure 5, which has been slightly deformed away from the real axis so that its incoming and outgoing legs do not overlap. The dashed lines represent a convenient choice of branch cuts of the square-root in the integrand. As before, the solid disks indicate the turning points and the open circles indicate the singularities.

The contribution to the contour integral of the small arc around the turning point vanishes because the integrand is zero there. The contributions of the remaining half-lines 


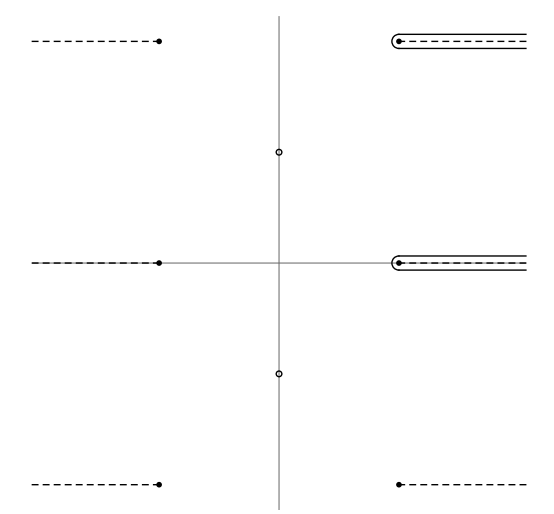

Figure 5. Reflected saddles with constant imaginary part. For $\eta$ real and less than $w$, there is a real saddle corresponding to a particle that comes in from $r \rightarrow \infty$, rolls partway up the hill until it stops at the turning point, and then rolls back out to infinity. The corresponding contour in the complex $r$-plane is pictured here, slightly deformed away from being pure real so that the incoming and outgoing segments of the contour do not overlap. Due to the $r \rightarrow r+\pi i$ shift symmetry of the complexified problem, one likewise has shifted contours with constant imaginary part $\pi N_{1}$, such as the second contour in the figure with $N_{1}=1$. The dashed lines represent branch cuts of the square-root in eq. (4.28).

above and below the real axis are identical because they sit on opposite sides of the branch cut and have opposite orientations, corresponding to the particle coming in from infinity and then going back out to infinity. Each of these two integrals contributes

$$
\int_{r_{+}}^{r(L)} \mathrm{d} r \sqrt{\eta^{2}+2 V(r)}=\eta \log (\eta)+\frac{w-\eta}{2} \log (w-\eta)-\frac{w+\eta}{2} \log (w+\eta)+\eta r(L),
$$

in the limit $L \rightarrow \infty$. Combined with the boundary term that cancels the linear divergence $\operatorname{\eta r}(L)$, we obtain the on-shell action

$$
\tilde{S}_{0}=2 \eta \log (\eta)+(w-\eta) \log (w-\eta)-(w+\eta) \log (w+\eta) .
$$

The contribution of this solution to the saddle-point expansion is then

$$
e^{-k \tilde{S}_{0}}=\eta^{-2 k \eta}(w+\eta)^{k(w+\eta)}(w-\eta)^{k(\eta-w)} .
$$

This accounts for the first half of eq. (4.21) with $0<\operatorname{Re}(\eta)<w$, leaving the three trigonometric factors still to be explained.

The simplest of these three factors to understand is $\csc (\pi k \eta)$. It is due to the shift symmetry of the potential [1]. Even when $\eta$ is real, one has complex solutions shifted by $\pi i N_{1}$, for any integer $N_{1}$. The contour with $N_{1}=1$ is also shown in figure 5 . Each of these shifted saddles has action $\tilde{S}_{0}-2 \pi i \eta N_{1}$, due to the shift of the boundary terms. One should not sum over all of them, however; the appropriate set depends on the sign of $\operatorname{Im}(\eta)$, which determines whether one obtains a convergent geometric series when $N_{1} \in \mathbb{Z}_{\geq 0}$ or when $N_{1} \in \mathbb{Z}_{\leq 0}$. For $\operatorname{Im}(\eta)>0$ one finds

$$
\sum_{N_{1} \in \mathbb{Z}_{\geq 0}} e^{2 \pi i k \eta N_{1}}=\frac{i}{2} e^{-\pi i k \eta} \csc (\pi k \eta)
$$


while for $\operatorname{Im}(\eta)<0$

$$
\sum_{N_{1} \in \mathbb{Z}_{\leq 0}} e^{2 \pi i k \eta N_{1}}=-\frac{i}{2} e^{\pi i k \eta} \csc (\pi k \eta) .
$$

In fact, since the reflection coefficient has poles on the real $\eta$-axis, one should always give $\eta$ a non-zero phase in computing the saddle-point expansion. We see here that depending on whether $\eta$ lies above or below the real axis, we must pick one or the other half-infinite set of shifted saddles. ${ }^{24}$

Next consider the factor of $\csc (\pi k(w-\eta))$, which accounts for the bound states of the infinite-space quantum mechanics. We now argue that it is the singular saddles that are responsible for this factor. This requires some explanation, since one ordinarily expects singular configurations to have infinite action and therefore to make no contribution to the functional integral. In contrast, the singular saddles in this complexified problem have finite action, and are essential to reproducing the correct semi-classical limit $[1,18]$.

Consider the singular saddle discussed in the previous sub-section, obtained as the $\rho_{0} \rightarrow \frac{\pi}{2 \eta}$ limit of eq. (4.30), with $N_{1}=0$. The contour is shown in figure 6a. As pictured in figure 4, on the real and imaginary slices $\operatorname{Im}(r)= \pm \frac{\pi}{2}$ and $\operatorname{Re}(r)=0$, the effective 1-dimensional potential is an infinite well in the neighborhood of the singular points. The speed of the particle subsequently diverges there. However, the remarkable feature of the complexified problem is that the divergent contributions to the action are equal-butopposite on the real and imaginary segments of the trajectory, the speed of the particle being pure real and pure imaginary in the two cases. One may therefore define the total action by a principal-value type limit.

More precisely, let $z=r-\frac{\pi i}{2}$ be a local coordinate in the neighborhood of the singularity at $r=\frac{\pi i}{2}$. From eq. (4.26), the potential has a double-pole there, $V(z)=\frac{w^{2}}{2} \frac{1}{z^{2}}+\mathcal{O}(1)$, and therefore the energy conservation equation in this neighborhood becomes

$$
\frac{\mathrm{d} z}{\mathrm{~d} \rho}=-\frac{w}{z}+\cdots
$$

the minus sign corresponding to the orientation chosen in figure 6a. The solution near the upper pole is then $z(\rho)=-i \sqrt{2 w\left(\rho+\rho_{1}\right)}$, hitting the pole at $\rho=-\rho_{1}$. The speedsquared is

$$
\left(\frac{\mathrm{d} z}{\mathrm{~d} \rho}\right)^{2}=-\frac{w}{2} \frac{1}{\rho+\rho_{1}}+\cdots
$$

\footnotetext{
${ }^{24}$ The same was true for the Gamma function example discussed earlier. $\Gamma(z)$ has poles on the negative real axis, and depending on whether $\operatorname{Im}(z)>0$ or $\operatorname{Im}(z)<0$, the integration contour is deformed into the steepest-descent contours for saddles in either the upper or lower-half $X$-plane.
} 


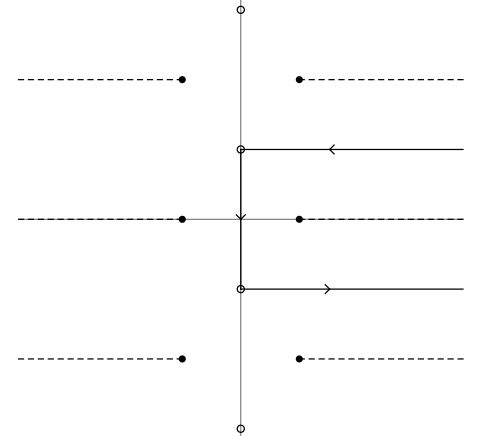

(a)

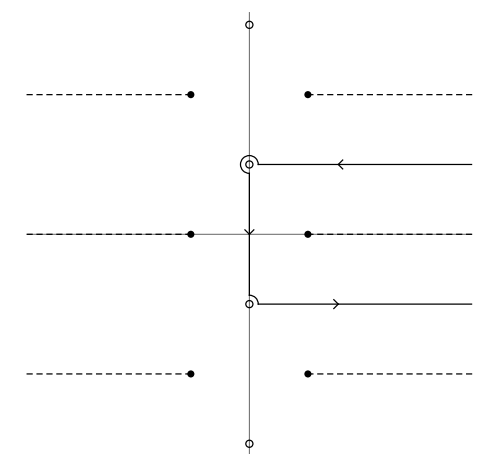

(b)

Figure 6. Singular contours. When the asymptotic imaginary part of the reflected contour shown in figure 3 reaches $\pm \frac{\pi}{2}$, the contour hits the poles of the potential and becomes singular (left). The singular contour must be deformed around the poles of the potential in order to define the action integral eq. (4.28). The action of the singular saddle then differs from eq. (4.33) by residues. The deformation is not unique, and one must sum over an appropriate set of singular saddles to reproduce the correct semi-classical limit. An example deformation is shown on the right, with action $\tilde{S}_{0}-2 \pi i w$.

The integrand of the on-shell action thus has a $\frac{1}{\rho}$ type singularity near $r=\frac{\pi i}{2}$, and the integral is ${ }^{25}$

$$
\int_{-\rho_{1}-\varepsilon}^{-\rho_{1}+\varepsilon} \mathrm{d} \rho\left(\frac{\mathrm{d} z}{\mathrm{~d} \rho}\right)^{2}=-\frac{w}{2}\left(\pi i+2 \pi i N_{2}\right)
$$

with $N_{2}$ an integer. The ambiguity in $2 \pi i \mathbb{Z}$ amounts to the choice of branch of $\log \rho=\int \frac{\mathrm{d} \rho}{\rho}$. One likewise has a $\frac{1}{\rho}$ singularity in the neighborhood of the lower pole. $\dot{r}^{2}$ for the complete singular solution with real $\eta$ is plotted in figure 7 .

In the formulation of the action as a contour integral in the $r$-plane, the ambiguity in the action of the singular saddle arises because one must deform the contour in figure $6 \mathrm{a}$ away from the poles at $r= \pm \frac{\pi i}{2}$, and the deformation is not unique. The same integral eq. (4.39) in the neighborhood of the pole may be written

$$
\int_{-\rho_{1}-\varepsilon}^{-\rho_{1}+\varepsilon} \mathrm{d} \rho\left(\frac{\mathrm{d} z}{\mathrm{~d} \rho}\right)^{2}=-\int_{\mathscr{C}_{\varepsilon}} \mathrm{d} z \frac{w}{z}
$$

\footnotetext{
${ }^{25}$ The integral $\int_{-a}^{a} \frac{\mathrm{d} \rho}{\rho}$ may be defined by continuing $\rho$ to the complex plane and deforming the contour off the real axis:

The integral over the counter-clockwise semi-circle about the pole is $\pi i$. Discarding it defines the principalvalue of the integral, which is zero in this symmetric case. Of course, the deformation of the contour is not unique. One could just as well have deformed it into a clockwise arc above the pole which would instead yield $-\pi i$, or an arc that encircles the pole any number of times. The integral is therefore only defined up to shifts by $2 \pi i \mathbb{Z}$, which is equivalently the ambiguity in the choice of branch of $\int \frac{\mathrm{d} \rho}{\rho}=\log (\rho)$.
} 


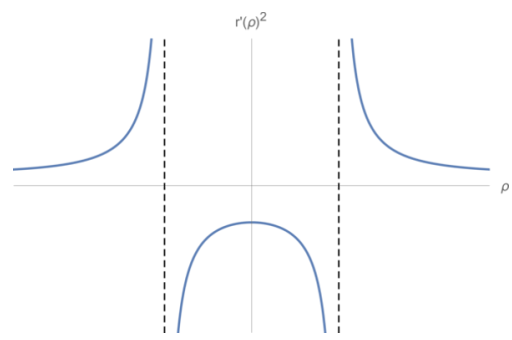

Figure 7. Singular speed-squared. The speed-squared $\dot{r}^{2}$ is plotted here for the singular saddle shown in figure 6a. It has $\frac{1}{\rho}$ type singularities where the contour hits the poles of the potential. The action, which is the integral of $\dot{r}^{2}$, is finite because $\int_{-\varepsilon}^{\varepsilon} \frac{\mathrm{d} \rho}{\rho}$ may be defined by continuation around the pole. The imaginary part of the integral is ambiguous up to shifts by $2 \pi i \mathbb{Z}$, however, corresponding to the choice of how to deform the contour around the poles.

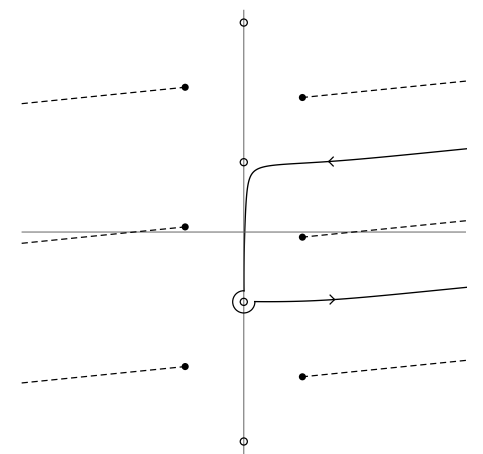

(a)

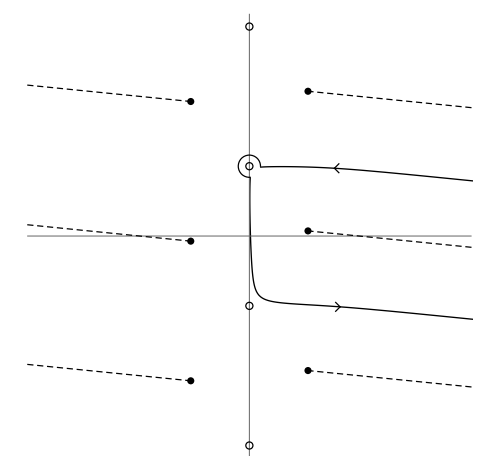

(b)

Figure 8. Singular reflected saddles $(\operatorname{Re}(\eta)<w)$. $\eta$ should be shifted off the real axis for the saddle-point expansion to be well-defined. Compared to figure $6, \eta$ has been given a small positive phase on the left and a small negative phase on the right.

where $\mathscr{C}_{\varepsilon}$ is a contour that avoids the pole. For example, the integral around the pole at $\frac{\pi i}{2}$ along the contour deformation shown in figure $6 \mathrm{~b}$ is $-\frac{3 \pi i}{2} w$, corresponding to $N_{2}=1$ in eq. (4.39).

The outcome of this discussion is that, in addition to the saddles accounted in eqs. (4.34), (4.35), and (4.36), one has singular saddles of finite action corresponding to contours in the $r$-plane that wrap the singularities an integer number of times and so differ from $\tilde{S}_{0}$ by residues. They may be thought of as asymptotic saddles in a fixed topological sector of the functional integral, where the singular points of the potential are excised from the plane, and the configuration space of maps $r:[-L, L] \rightarrow\{\mathbb{C}-$ poles $\}$ is divided into homotopy classes labeled by their winding numbers around the punctures. As the deformations of the contour around the poles shrink away, one asymptotically approaches an exact solution of the equations of motion, whose action differs from $\tilde{S}_{0}$ in its imaginary part.

A singular saddle that wraps $N_{2}$ times around the pole at $r=\frac{\pi i}{2}+\pi i N_{1}$ has action $\tilde{S}_{0}-2 \pi i \eta N_{1}-2 \pi i w N_{2}$. Figures 8 a and 8 b illustrate such saddles with $N_{1}=-1$ and $0, N_{2}=$ -1 and 1 , and $\eta$ having a small positive and negative phase, respectively. By summing over saddles with action of the form $\tilde{S}_{0}-2 \pi i \eta\left(N_{1}+N_{2}\right)+2 \pi i w N_{2}=\widetilde{S}_{0}-2 \pi i \eta N_{1}-2 \pi i(\eta-w) N_{2}$, 
one may obtain both factors of $\csc (\pi k \eta)$ and $\csc (\pi k(w-\eta))$ required in eq. (4.21). We give the precise list of saddles momentarily.

Finally, one must account for the factor of $\sin (\pi k w)$ in eq. (4.21). It is again associated to the singular saddles, but it is qualitatively different from the csc factors, corresponding to a 2-fold degeneracy of saddles rather than an infinite geometric series. Written in the form

$$
\sin (\pi k w) \propto e^{\pi i k w}\left(1-e^{-2 \pi i k w}\right),
$$

we see that we must sum over two sets of singular saddles, identical except that each contour in one set winds an extra time around the pole. The relative minus sign is due to the orientation of the integration contours, which we will not attempt to determine.

Having explained the mechanism by which each factor in eq. (4.21) comes about, let us finally give the list of saddles that reproduces the semi-classical limit for $0<\operatorname{Re}(\eta)<w$.

For $\operatorname{Im}(\eta)>0$, the contributing saddles have action

$$
\begin{array}{ll}
\tilde{S}_{N_{1} N_{2}}=\tilde{S}_{0}-2 \pi i \eta\left(N_{1}+N_{2}\right)+2 \pi i w N_{2}, & N_{1}=0,1,2, \ldots \\
& N_{2}=1,2,3, \ldots,
\end{array}
$$

corresponding to a contour that wraps $N_{2}$ times around the pole at $r=-\frac{\pi i}{2}+\pi i\left(N_{1}+N_{2}\right)$. Note that $N_{1}+N_{2} \geq 1$, meaning that all the contours are in the upper-half plane. In addition, one has a second set of saddles with action

$$
\begin{array}{ll}
\tilde{S}_{N_{1} N_{2}}^{\prime}=\tilde{S}_{0}-2 \pi i \eta\left(N_{1}+N_{2}\right)+2 \pi i w\left(N_{2}-1\right), & N_{1}=0,1,2, \ldots \\
& N_{2}=1,2,3, \ldots
\end{array}
$$

which wrap $N_{2}-1$ times instead. The two sets are weighted with a relative minus sign.

Their contribution to the saddle-point expansion yields at leading order

$$
\begin{aligned}
\sum_{\substack{N_{1} \in \mathbb{Z}_{\geq 0} \\
N_{2} \in \mathbb{Z}_{\geq 1}}}\left(e^{-k \tilde{S}_{N_{1} N_{2}}}-e^{-k \tilde{S}_{N_{1} N_{2}}^{\prime}}\right) \\
\quad=e^{-k \tilde{S}_{0}} \sum_{N_{1} \in \mathbb{Z}_{\geq 0}} e^{2 \pi i k \eta N_{1}} \sum_{N_{2} \in \mathbb{Z}_{\geq 1}} e^{2 \pi i k(\eta-w) N_{2}}\left(1-e^{2 \pi i k w}\right) \\
\propto \eta^{-2 k \eta}(w+\eta)^{k(w+\eta)}(w-\eta)^{k(\eta-w)} \sin (\pi k w) \csc (\pi k \eta) \csc (\pi k(w-\eta)),
\end{aligned}
$$

reproducing eq. (4.21). Note that the geometric series converge for $\operatorname{Im}(\eta)>0$. For $\operatorname{Im}(\eta)<$ 0 , the required sum is instead

$$
e^{-k \tilde{S}_{0}} \sum_{N_{1} \in \mathbb{Z}_{\leq 0}} e^{2 \pi i k \eta N_{1}} \sum_{N_{2} \in \mathbb{Z}_{\leq-1}} e^{2 \pi i k(\eta-w) N_{2}}\left(1-e^{-2 \pi i k w}\right),
$$

with the same result. These are contours that wrap $N_{2}$ or $N_{2}+1$ times around the pole at $r=\frac{\pi i}{2}+\pi i\left(N_{1}+N_{2}\right)$. Note that $N_{1}+N_{2} \leq-1$ implies all of the contours are in the lower-half plane.

As forewarned at the beginning of this section, we have not attempted to explain why these are the saddles that contribute to the functional integral, but merely demonstrated 


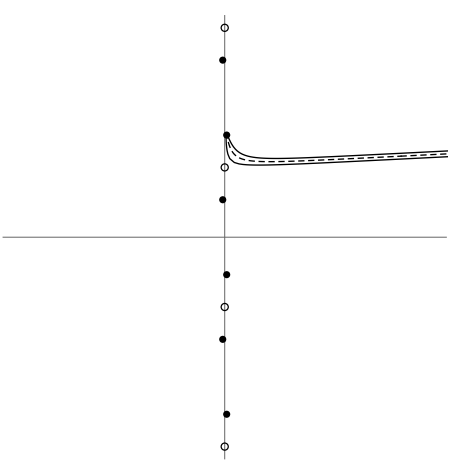

(a)

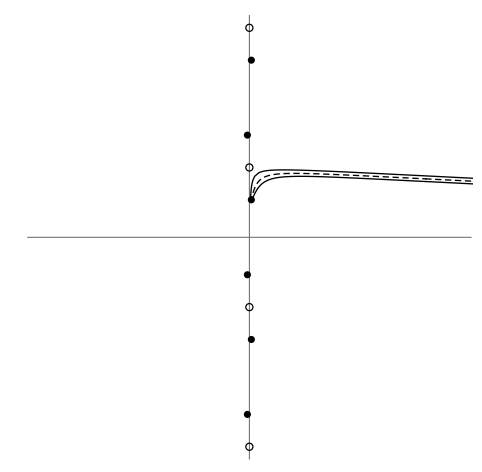

(b)

Figure 9. Reflected saddles $(\operatorname{Re}(\eta)>w)$. When $\eta$ is real and greater than $w$, the only reflected solutions are singular. But one finds non-singular solutions for complex $\eta$. On the left $\eta$ has a small positive phase and on the right it has a small negative phase. The contour is deflected in opposite directions by the pole in the two cases.

that this is the necessary list to reproduce the semi-classical limit of the exact answer. In fact, we take this list as the definition of the contour of the functional integral that computes the reflection coefficient for the quantum mechanics, being given by the sum of the corresponding steepest-descent contours.

So far we have considered the case $0<\operatorname{Re}(\eta)<w$. Next suppose that $\operatorname{Re}(\eta)>w$. In eq. (4.21), the bound state factor now disappears, because $\frac{\eta^{2}}{2}$ exceeds the height of the potential. As discussed previously, in light of this abrupt change in the asymptotic expansion of the reflection coefficient, we expect that $\operatorname{Re}(\eta)=w$ corresponds to a Stokes wall, and that the set of contributing saddles jumps for $\operatorname{Re}(\eta)>w$.

When $\eta$ is real and larger than $w$, one no longer has a real solution of the equations of motion. The energy of the particle is greater than the height of the potential hill and it rolls over from $r \rightarrow \infty$ to $r \rightarrow-\infty$, satisfying the boundary conditions for transmission rather than reflection. The only reflected trajectories with $\eta$ real and greater than $w$ are singular. Once again, $\eta$ should be given a phase, in which case one finds non-singular trajectories. Figures $9 \mathrm{a}$ and $9 \mathrm{~b}$ illustrate reflected solutions for $\eta$ with a small positive and a small negative phase. As is by now familiar, the set of contributing saddles will depend on whether $\eta$ lies above or below the real axis.

The action for the contour with $\operatorname{Im}(\eta)>0$ pictured in figure $9 \mathrm{a}$ is

$$
\tilde{S}_{0}=2 \eta \log (\eta)-(\eta+w) \log (\eta+w)-(\eta-w) \log (\eta-w)-\pi i(\eta+w) .
$$

The contributing saddles for $\operatorname{Im}(\eta)>0$ are the shifted saddles of this form in the upper-half plane,

$$
\tilde{S}_{N_{1}}=\tilde{S}_{0}-2 \pi i \eta N_{1}, \quad N_{1}=0,1,2, \ldots,
$$

as well as a second set that wraps the pole at $r=\frac{\pi i}{2}+\pi i N_{1}$ once,

$$
\tilde{S}_{N_{1}}^{\prime}=\tilde{S}_{N_{1}}+2 \pi i w, \quad N_{1}=0,1,2, \ldots
$$




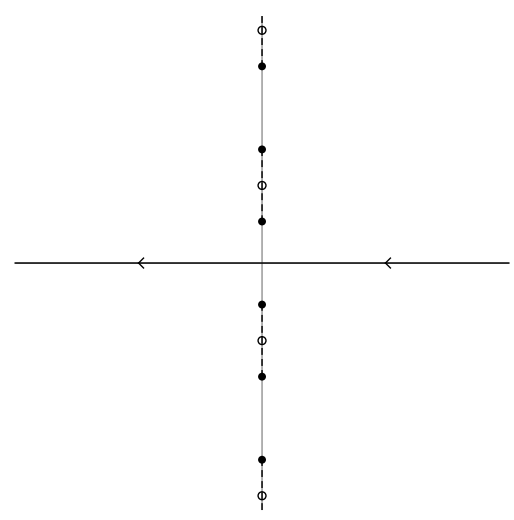

Figure 10. Transmitted saddle. $(\eta>w)$. For $\eta$ real and greater than $w$, there is a real saddle for the transmission coefficient, corresponding to a particle that rolls over the inverted potential from $r \rightarrow \infty$ to $r \rightarrow-\infty$.

The two sets are weighted with a relative minus sign, for a total of

$$
\begin{aligned}
\sum_{N_{1} \in \mathbb{Z}_{\geq 0}}\left(e^{-k \tilde{S}_{N_{1}}}-e^{-k \tilde{S}_{N_{1}}^{\prime}}\right) & =e^{-k \tilde{S}_{0}} \sum_{N_{1} \in \mathbb{Z}_{\geq 0}} e^{2 \pi i k \eta N_{1}}\left(1-e^{-2 \pi i k w}\right) \\
& \propto \eta^{-2 k \eta}(\eta+w)^{k(\eta+w)}(\eta-w)^{k(\eta-w)} \sin (\pi k w) \csc (\pi k \eta) .
\end{aligned}
$$

For $\operatorname{Im}(\eta)<0$, the necessary saddles are of the form in figure $9 \mathrm{~b}$, but in the lower-half plane. The action for the contour in figure $9 \mathrm{~b}$ is

$$
\tilde{S}_{0}=2 \eta \log (\eta)-(\eta+w) \log (\eta+w)-(\eta-w) \log (\eta-w)-\pi i(\eta-w),
$$

and the saddle-point expansion is

$$
e^{-k \tilde{S}_{0}}\left(\sum_{N_{1} \in \mathbb{Z}_{\leq-1}} e^{2 \pi i k \eta N_{1}}\right)\left(1-e^{2 \pi i k w}\right) .
$$

This completes the saddle-point expansion of the reflection coefficient for the infinite-space quantum mechanics. Next we turn to the transmission coefficient.

\subsubsection{Transmission coefficient on the complex $r$-plane}

In this sub-section we compute the saddle-point expansion of the transmission coefficient for the infinite-space quantum mechanics. The action is as in eq. (4.28), but with $r(L) \rightarrow$ $-r(L)$ so that the velocity at late times is fixed to $-\eta$ rather than $\eta$ :

$$
\tilde{S}[r]=\int_{\mathscr{C}} \mathrm{d} r \sqrt{\eta^{2}+2 V(r)}-\eta(-r(L)+r(-L)) .
$$

The simplest saddle of eq. (4.52) is the real trajectory of a particle with $\eta$ real and greater than $w$ that rolls over the potential hill, pictured in figure 10 .

The action for this saddle is ${ }^{26}$

$$
\tilde{S}_{0}=2 \eta \log (\eta)-(\eta+w) \log (\eta+w)-(\eta-w) \log (\eta-w),
$$

\footnotetext{
${ }^{26}$ Note that the appropriate branch of the square-root in the integrand of the action should be negative on the real axis, the velocity of the particle always being to the left for this trajectory.
} 


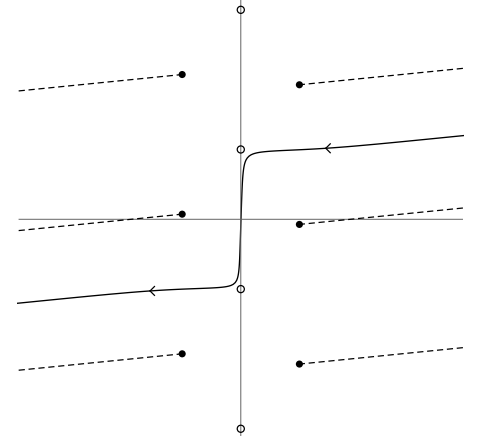

(a)

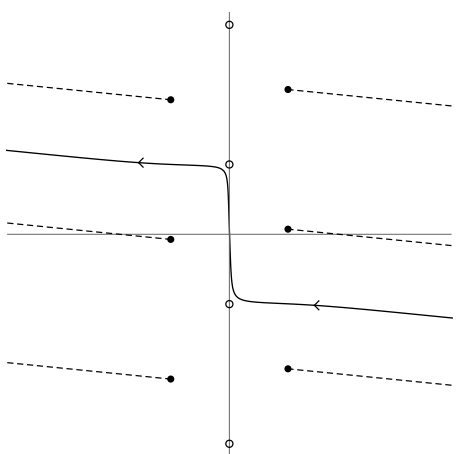

(b)

Figure 11. Transmitted saddles $(\operatorname{Re}(\eta)<w)$. When $\eta$ is real and less than $w$, the only transmitted saddles are singular. Non-singular solutions are found for complex $\eta$. On the left $\eta$ has a small positive phase and on the right it has a small negative phase.

yielding

$$
e^{-k \tilde{S}_{0}}=\eta^{-2 k \eta}(\eta+w)^{k(\eta+w)}(\eta-w)^{k(\eta-w)} .
$$

This reproduces the transmission coefficient eq. (4.22) for $\operatorname{Re}(\eta)>w$. Note that although one again has shifted saddles with constant imaginary part $\pi N_{1}$, the action is invariant under the shift. For the same reason, note that the functional integral over real $r$ no longer diverges for the transmission coefficient. Indeed, given that the semi-classical limit of the transmission coefficient is reproduced by a single real saddle for real $\eta$, we expect that the contour of integration is real in this case.

When $\eta$ is real and less than $w$, the only saddles for the transmission coefficient are singular. Non-singular saddles are obtained for complex $\eta$, as pictured in figure 11.

The action for the contour pictured in figure 11a, where $\operatorname{Im}(\eta)>0$, is

$$
\tilde{S}_{0}=2 \eta \log (\eta)-(w+\eta) \log (w+\eta)+(w-\eta) \log (w-\eta)+\pi i(w-\eta),
$$

which gives

$$
e^{-k \tilde{S}_{0}}=e^{-\pi i k(w-\eta)} \eta^{-2 k \eta}(\eta+w)^{k(\eta+w)}(w-\eta)^{k(\eta-w)} .
$$

The necessary sum is now

$$
e^{-k \tilde{S}_{0}} \sum_{N \in \mathbb{Z} \geq 0} e^{2 \pi i k(\eta-w) N} \propto \eta^{-2 k \eta}(\eta+w)^{k(\eta+w)}(w-\eta)^{k(\eta-w)} \csc (\pi k(w-\eta)),
$$

corresponding to saddles with $\tilde{S}_{N}=\tilde{S}_{0}-2 \pi i \eta N+2 \pi i w N$. As in the reflected case, the shift by $2 \pi i w N$ is accounted for by singular saddles that wrap the pole $N$ times. The shift by $2 \pi i \eta N$ was previously explained by the change in the boundary action under $r \rightarrow r+\pi i N$. As noted a moment ago, however, the boundary action for transmission is invariant under this shift.

Instead, one must consider contours with only one end shifted by $2 \pi i N$, as pictured in figure 12. The integral along the shifted contour is identical to that of figure 11a; their actions differ only by the shift of the boundary term. By summing over contours of 


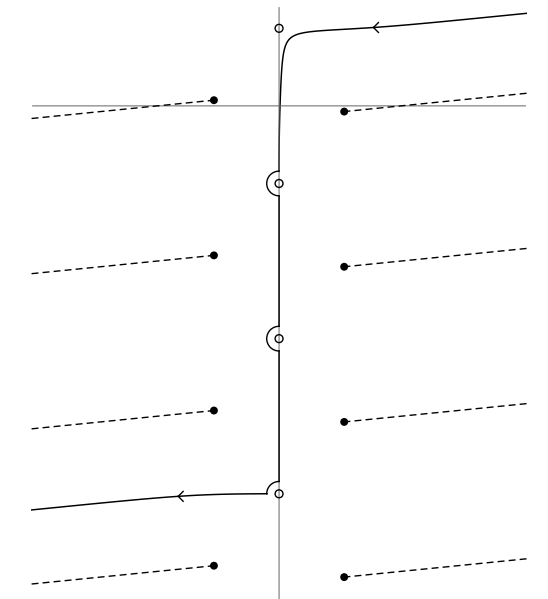

Figure 12. Shifted transmitted contour. When $\operatorname{Re}(\eta)<w$, one must sum over contours with $r(L) \rightarrow r(L)-2 \pi i N$ in order to reproduce the semi-classical limit of the transmission coefficient.

this form, where $r(L) \rightarrow r(L)-2 \pi i N$, together with the $N$-fold wrapping, we obtain the required lattice of actions for eq. (4.57).

For $\operatorname{Im}(\eta)<0$, the action of the saddle pictured in figure $11 \mathrm{~b}$ is

$$
\tilde{S}_{0}=2 \eta \log (\eta)-(w+\eta) \log (w+\eta)+(w-\eta) \log (w-\eta)-\pi i(w-\eta),
$$

and the saddle-point expansion is

$$
e^{-k \tilde{S}_{0}} \sum_{N \in \mathbb{Z}_{\leq 0}} e^{2 \pi i k(\eta-w) N}
$$

One has analogous contours as in figure 12, now with $r(L) \rightarrow r(L)-2 \pi i N$ shifted upward.

We do not have a clear rationale for why contours of this form contribute to the saddle-point expansion. Although the contour pictured in figure 12 is constructed out of the same components as the singular saddles discussed previously, it is not obtained from a limit of smooth saddles, which are always confined to an interval of width $\pi i$. For now we merely observe that this is the set which reproduces the semi-classical limit of the exact transmission coefficient, and leave it as an open question to better understand the rules for determining the set of contours that contribute to the saddle-point expansion.

\subsection{Bound states and the cigar wrapping saddle}

Let us now return to the problem of identifying an asymptotic condition for a bound state insertion in the functional integral mentioned in section 3.3. The asymptotic conditions in eq. (3.31) describing an operator insertion $\mathcal{O}_{j n w}$ in the far past on the cylinder assume a generic value of $j$, with $\operatorname{Re}(j)>\frac{1}{2}$, on which $R(j, n, w)$ is non-singular. On the bound state spectrum $j_{N}, R$ has simples poles, and one must be more careful. In that case, it is the reflected term in eq. (3.18) that dominates in the weak coupling region, $\frac{1}{R} \mathcal{O}_{j n w} \rightarrow \mathcal{V}_{Q j, p_{\mathrm{L}}, p_{\mathrm{R}}}$, and the free field asymptotic condition flips sign,

$$
r \stackrel{\rho \rightarrow-\infty}{\longrightarrow} \frac{2}{k}\left(j-\frac{1}{2}\right) \rho
$$


This asymptotic condition maps the string out of the free field region and is inconsistent. This agrees with the fact that the bound states are normalizable, and do not extend out to infinity in $r$.

The linear solution, pictured in figure 13, is a saddle for the two-point function of the linear-dilaton primaries $e^{-2(1-j) r}$ and $e^{-2 j r}$, sending the string to $r \rightarrow \infty$ and $r \rightarrow$ $-\infty$ in the neighborhoods of the two respective operators. In the cigar, of course, the geometry ends at $r=0$, and in the vicinity of the tip the free cylinder equations of motion are modified by the curvature of the cigar. One would like to understand how the free trajectory is corrected once the string leaves the free field region, and thereby obtain an asymptotic condition for the bound state insertion. We will argue that the string worldsheet asymptotically wraps the tip of the cigar.

Because the neighborhood of the bound state insertion is mapped out of the free field region, the large $r$ expansion $e^{-2 j r}$ of the operator is insufficient to determine the requisite asymptotic condition. The radial dependence of the vertex operator on the full cigar was obtained in $[3]:{ }^{27,28}$

$$
\begin{aligned}
& \frac{4^{j-1}}{\Gamma(2 j-1)} \frac{\Gamma(j+m) \Gamma(j-\bar{m})}{\Gamma(1+m-\bar{m})} \\
& \times \sinh ^{m-\bar{m}}(r) \cosh ^{-(m+\bar{m})}(r)_{2} F_{1}\left(j-\bar{m}, 1-j-\bar{m} ; 1+m-\bar{m} ;-\sinh ^{2}(r)\right) \\
& \quad \stackrel{r \rightarrow \infty}{\longrightarrow} e^{-2(1-j) r}+4^{2 j-1} \frac{\Gamma(1-2 j)}{\Gamma(2 j-1)} \frac{\Gamma(j+m) \Gamma(j-\bar{m})}{\Gamma(1-j+m) \Gamma(1-j-\bar{m})} e^{-2 j r},
\end{aligned}
$$

where $m=\frac{1}{2}(-k w+n)$ and $\bar{m}=\frac{1}{2}(-k w-n)$. Note that the reflection coefficient reproduces the second line of eq. (3.19). ${ }^{29}$

In the parent $\mathrm{SL}(2, \mathbb{R})_{k}$ WZW model prior to the coset, $m$ and $\bar{m}$ correspond to the eigenvalues ${ }^{30}$ of the $\widehat{\mathfrak{s l}}_{k}(2, \mathbb{R})_{\mathrm{L}} \oplus \widehat{\mathfrak{s l}}_{k}(2, \mathbb{R})_{\mathrm{R}}$ current algebra zero-modes $J_{0}^{3}$ and $\bar{J}_{0}^{3} \cdot m-\bar{m}=n$ is the quantized angular momentum around the $\mathrm{AdS}_{3}$ cylinder, and $m+\bar{m}=-k w$ is the projection condition for the coset, which gauges $J^{3}+\bar{J}^{3}[4]$. The poles of $\Gamma(j+m)$ in the reflection coefficient correspond to states in highest-weight discrete series representations of $\mathfrak{s l}(2, \mathbb{R}), m \in-j-\mathbb{N}$, while the poles of $\Gamma(j-\bar{m})$ correspond to states in lowest-weight discrete series representations, $\bar{m} \in j+\mathbb{N}[4]$. For $w<0$, the coset bound states descend from the lowest-weight discrete series states, while for $w>0$ they descend from the highestweight states. ${ }^{31}$ Likewise, in eq. (3.22) we saw that the coset bound states appear in the poles of $\Gamma\left(j+\frac{|n| \pm k w}{2}\right)$, depending on the sign of $w$.

\footnotetext{
${ }^{27}$ This expression holds for $m \geq \bar{m}$ (i.e. $n \geq 0$ ). Note that the reflection coefficient is invariant under $w \rightarrow-w$. For $m \leq \bar{m}$ one sends $m \rightarrow-m$ and $\bar{m} \rightarrow-\bar{m}$, which is equivalent to flipping the signs of $n$ and $w$. Then it is the absolute value of $n$ that appears in the reflection coefficient, as in eq. (3.19).

${ }^{28}$ To obtain the wavefunction, one would multiply by $\cosh (r)$ as described in section 3.2 .

${ }^{29}$ It is unclear to us if the missing factor from the first line of eq. (3.19), which is of order one in the large $k$ limit, is due to a non-perturbative correction to the cigar background as has been suggested in the literature [30-32], or if it is a perturbative correction.

${ }^{30}$ More precisely, their eigenvalues are $m+\frac{k w}{2}$ and $\bar{m}+\frac{k w}{2}$.

${ }^{31}$ The reason being that $m, \bar{m} \in j+\mathbb{N}$ are positive in lowest-weight representations since $j$ is positive, while $m, \bar{m} \in-j-\mathbb{N}$ are negative in highest-weight representations. To satisfy the projection $m+\bar{m}=-k w$ therefore requires a highest-weight representation for $w>0$ and a lowest-weight representation for $w<0$.
} 
Recalling that the hypergeometric series terminates when its first or second argument is a non-positive integer, we find that the hypergeometric function is a finite order polynomial in $\sinh ^{2}(r)$ on the bound state spectrum. On the lowest-weight states, $j-\bar{m}=-N$, this is evident from eq. (4.61), where the hypergeometric function yields an order $N$ polynomial in $\sinh ^{2}(r)$. On the highest-weight states, $j+m=-N$, it becomes evident after applying the hypergeometric fractional transformation rule

$$
\begin{aligned}
& { }_{2} F_{1}\left(j-\bar{m}, 1-j-\bar{m} ; 1+m-\bar{m} ;-\sinh ^{2}(r)\right) \\
& \quad=\cosh ^{2(m+\bar{m})}(r){ }_{2} F_{1}\left(j+m, 1-j+m ; 1+m-\bar{m} ;-\sinh ^{2}(r)\right) .
\end{aligned}
$$

Let us again restrict our focus to the pure-winding sector, $n=0$. On the bound state spectrum $j_{N}=\frac{k|w|}{2}-N$, one obtains

$$
\mathcal{O}_{j_{N}, n=0, w} \propto \operatorname{sech}^{k|w|}(r)_{2} F_{1}\left(-N,-k|w|+N+1 ; 1 ;-\sinh ^{2}(r)\right) .
$$

For $N=0$ the hypergeometric function is 1 , for $N=1$ it is $1+(-k|w|+2) \sinh ^{2}(r)$, and so on.

These operators consist of a heavy factor $\operatorname{sech}^{k|w|}(r)$, which enters at the same order as the leading terms in the action, times a light factor, which is sub-leading. The heavy factor inserts a source in the leading equations of motion and therefore affects the form of the saddles. The light factor, by constrast, is merely evaluated on the leading saddles and contributes to the sub-leading correction in the saddle-point expansion. Moreover, since the heavy factor is independent of $N$, the behavior of the saddle for any bound state insertion is independent of $N$.

We have assumed here that $N$ is of order one in the large $k$ limit, else the order $N$ polynomial in $\sinh ^{2}(r)$ would no longer be a light operator. In view of the upper-bound $j<\frac{k-1}{2}$ on the physical spectrum, we will moreover choose $|w|=1$, such that the bound may be satisfied for $N$ of order one. Indeed, our principal interest is in the sine-Liouville operator eq. (3.24), which is the sum of operators with $j=\frac{k}{2}-1$ and $w= \pm 1$. We will focus below on $w=-1$; the case $w=1$ is analogous.

Since the asymptotic condition for $\mathcal{O}_{j=\frac{k}{2}-N, n=0, w=-1}$ is independent of $N$, we may set $N=0$. This state is not part of the physical spectrum, of course. In fact, it is rather special in the continued space of states; it is in a sense a reflection of the identity operator [4]. Note first of all that its conformal weight is zero. In the coset construction from $\operatorname{SL}(2, \mathbb{R})_{k}$, $\mathcal{O}_{j=\frac{k}{2}, n=0, w=-1}$ descends from the state

$$
\left|j=\frac{k}{2}, m=\frac{k}{2}, \bar{m}=\frac{k}{2} ; w=-1\right\rangle \in \widehat{D}_{\frac{k}{2}}^{+, w=-1} \otimes \widehat{D}_{\frac{k}{2}}^{+, w=-1} .
$$

Here, $D_{\frac{k}{2}}^{+}$denotes the spin $j=\frac{k}{2}$ lowest-weight discrete series representation of the global $\mathfrak{s l}(2, \mathbb{R})$ sub-algebra, $\widehat{D}_{\frac{k}{2}}^{+}$denotes the $\widehat{\mathfrak{s l}}_{k}(2, \mathbb{R})$ current algebra representation built upon it, and $\widehat{D}_{\frac{k}{2}}^{+, w=-1}$ denotes the spectral-flowed current algebra representation by minus one unit. For the details of these representations, see [4]. 


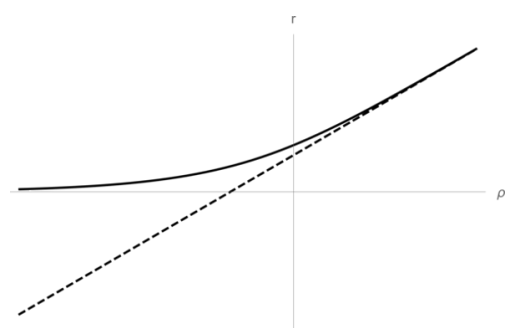

Figure 13. The cigar wrapping saddle. When $j=j_{N}$ lies on the bound state spectrum eq. (3.9b), the reflection coefficient is singular, and it is the otherwise sub-leading term $e^{-2 j r}$ in eq. (3.18) that describes the operator $\mathcal{O}_{j n w}$ in the asymptotic region. The free field Green function, shown by the dashed line, maps the string out of the free field region and must be modified. The complete solution for $|w|=1$ and $n=0$ is $\theta= \pm \phi, r=\sinh ^{-1}\left(e^{\rho}\right)$. The neighborhood of the bound state insertion wraps the tip of the cigar, with $r \rightarrow e^{\rho}$ asymptotically approaching $r=0$. The leading saddle is independent of $N$, which enters in the sub-leading correction to the saddle-point approximation.

$\left|j=\frac{k}{2}, m=\frac{k}{2}, \bar{m}=\frac{k}{2}\right\rangle$ is known as the spectral flow operator $[4,6]$ because its product with another operator imparts one unit of spectral flow. By flowing this state backward by one unit as in eq. (4.64), one obtains a trivial operator of $J_{0}^{3}, \bar{J}_{0}^{3}$, and conformal weight zero. Under the isomorphism $\widehat{D}_{j}^{+, w} \simeq \widehat{D}_{\frac{k}{2}-j}^{-, w+1}$ of spectral-flowed discrete series representations, it maps to the trivial highest-weight state $\left|j^{\prime}=0, m^{\prime}=0, \bar{m}^{\prime}=0 ; w^{\prime}=0\right\rangle$.

To understand the asymptotic condition associated to this operator, return to the cigar quantum mechanics eq. (4.14), obtained after choosing a pure-winding configuration $\theta=\phi$ . The inverted potential $-V(r)=\frac{1}{2} \operatorname{sech}^{2}(r)$ is a hill of height $\frac{1}{2}$, as shown in figure 2. For generic real values of $\eta<1$, one obtained real solutions describing a particle that comes in from infinity with speed $\eta$, rolls partway up the hill to a height of $\frac{1}{2} \eta^{2}$, and then rolls back to infinity. For $\eta=1$, corresponding to $j=\frac{k}{2}$, the particle has just enough energy to asymptotically approach the top of the potential at $r=0$. It does not return to infinity, but rather wraps the tip of the cigar, taking infinite time to do so.

This cigar wrapping solution is

$$
r(\rho)=\sinh ^{-1}\left(e^{\rho}\right)
$$

shown in figure 13, with limiting behavior

$$
r(\rho) \rightarrow \begin{cases}e^{\rho} & \rho \rightarrow-\infty \\ \rho & \rho \rightarrow \infty\end{cases}
$$

As expected from eq. (4.60) with $j=\frac{k}{2}$, in the asymptotic region the solution approaches the free field Green function $r \rightarrow \rho$. Eq. (4.65) gives the completion of the solution beyond the free field region. The asymptotic condition

$$
\begin{aligned}
& r \stackrel{\rho \rightarrow-\infty}{\longrightarrow} e^{\rho} \\
& \theta \stackrel{\rho \rightarrow-\infty}{\longrightarrow} \phi,
\end{aligned}
$$


describes a string that wraps the tip of the cigar. Since the bound state operators for $N \neq 0$ differ at sub-leading order, we claim that this is the appropriate asymptotic condition for any $N$ of order one. Observe that this is simply the holomorphic map that sends the worldsheet coordinate $z=e^{\rho} e^{i \phi}$ to the target coordinate $Z=r e^{i \theta}$ in the neighborhood of the tip of the cigar, where the geometry is $\mathbb{R}^{2}$. As usual, one may shift $\rho$ by a continuous modulus $i \rho_{0}$ that changes the angle at which the trajectory approaches the origin in the complex $r$-plane, and one may moreover consider solutions shifted by $\pi i \mathbb{Z}$.

Note that $\dot{r} \rightarrow r$ as $\rho \rightarrow-\infty$ and $\dot{r} \rightarrow 1$ as $\rho \rightarrow \infty$. This configuration is therefore a saddle of the action

$$
\begin{aligned}
S= & \frac{k}{4 \pi} \int_{-L}^{L} \mathrm{~d} \rho \int_{0}^{2 \pi} \mathrm{d} \phi\left(\left(\partial_{\rho} r\right)^{2}+\left(\partial_{\phi} r\right)^{2}+\tanh ^{2}(r)\left(\left(\partial_{\rho} \theta\right)^{2}+\left(\partial_{\phi} \theta\right)^{2}\right)\right) \\
& +k \int_{0}^{2 \pi} \frac{\mathrm{d} \phi}{2 \pi}\left(-\left.r\right|_{\rho=L}+\left.\frac{1}{2} r^{2}\right|_{\rho=-L}\right) \\
& +k \int_{0}^{2 \pi} \frac{\mathrm{d} \phi}{2 \pi}\left(\sigma_{+}\left(\left.\partial_{\phi} \theta\right|_{\rho=L}-1\right)+\sigma_{-}\left(\left.\partial_{\phi} \theta\right|_{\rho=-L}-1\right)\right)+\mathcal{O}\left(k^{0}\right),
\end{aligned}
$$

with radial boundary equations of motion

$$
\begin{gathered}
\left.\partial_{\rho} r\right|_{\rho=L}=1 \\
\left.\partial_{\rho} r\right|_{\rho=-L}=\left.r\right|_{\rho=-L} .
\end{gathered}
$$

The on-shell action is $S=-k \log (2)$.

Thus, one may interpret the cigar wrapping configuration as a saddle for the two-point function of $\mathcal{O}_{j=\frac{k}{2}-N, n=0, w= \pm 1}$. In the special case when $N=0$, this is a trivial operator. Then the $r^{2}$ boundary term yields the identity operator in the limit that it shrinks away, and one may alternatively interpret the configuration as a saddle for the one-point function. For $N \neq 0$, one inserts the light factor of the operator at the boundary and the insertion becomes non-trivial.

Since the reflection coefficient is singular, the sum over complex saddles may diverge with the free field boundary condition specified by the linear boundary term at $\rho=L$. Our primary interest is not in the cigar wrapping saddle itself, however, but in the tip wrapping asymptotic condition $\dot{r} \rightarrow r$ for the bound states. 
With the asymptotic condition in hand, one may use it to compute the saddle-point expansion of correlation functions with bound state insertions. We will not pursue any such calculations here. ${ }^{32}$ The two-point function of such operators amounts to a choice of normalization, and the saddle-point expansion of a three-point function is considerably more challenging. Some exact three-point functions with bound state insertions have been computed exactly, however, such as the correlator of $\mathcal{O}_{j_{1}=\frac{k}{2}-N, n_{1}=0, w_{1}=-1}, \mathcal{O}_{j_{2}=\frac{k w_{2}}{2}, n_{2}=0, w_{2}}$, and $\mathcal{O}_{j_{3}, n_{3}=0, w_{3}=1-w_{2}}$ in [33]. The result is independent of $N$ at leading order in the large $k$ limit, in support of the semi-classical picture we have described above.

It would be very interesting to explore the implications of this semi-classical definition of the bound state operators for the infinitesimal version of the FZZ duality, which relates the sine-Liouville operator to a deformation of the cigar that shifts the value of the dilaton at the tip [27, 34]. In particular, in the Lorentzian continuation of the duality [35, 36] that we propose in [34], the above prescription describes a string that crosses the black hole horizon, as $r=0$ is the bifurcation point. It is interesting that in the presence of horizons such additional operators are required beyond the ordinary scattering states to close the OPE, and we hope to use these semi-classical methods to more precisely understand string theory in Rindler space [37] and the stringy description of horizon entropy [38].

\subsection{Scattering states}

Above we have focused on the semi-classical limit of the reflection coefficient for purewinding operators $\mathcal{O}_{j, n=0, w}$, which includes the simplest bound states in the $\mathrm{SL}(2, \mathbb{R})_{k} / \mathrm{U}(1)$ spectrum. We close this section by briefly commenting on the analogous calculation for the pure-momentum operators $\mathcal{O}_{j, n, w=0}$, whose continuation under $n \rightarrow i E$ yield scattering states on the Lorentzian black hole background, with spatial momentum $s$ determined by the linear-dilaton momentum $j=\frac{1}{2}(1+i s)$ [3]. The saddle-point expansions in the two sectors are closely parallel because the pure-winding and pure-momentum operators are

\footnotetext{
${ }^{32}$ We conjecture, however, that the relevant saddles for the two-point function of $\mathcal{O}_{j=\frac{k}{2}-N, n=0, w= \pm 1}$ computed with the tip wrapping asymptotic conditions are given by trajectories that asymptote between neighboring maxima of the inverted potential on the complex $r$ plane, such as $r=0$ and $r=\pi i$. On the imaginary axis, pictured in figure $4 \mathrm{~b}$, the potential is singular at $r=\pi i / 2$. However, in the $k$-corrected potential [3],

$$
V(r)=\frac{1}{2}\left(\frac{1}{\operatorname{coth}^{2}(r)-\frac{2}{k}}-\frac{k}{k-2}\right),
$$

the double pole at $r=\pi i / 2$ splits into a pair of simple poles at $\frac{\pi i}{2} \pm \sqrt{\frac{2}{k}}+\mathcal{O}\left(k^{-3 / 2}\right)$. Then the potential on the imaginary axis is regular, and one may consider, for example, a trajectory that asymptotes between $r=0$ in the far past and $r=\pi i$ in the far future:
}

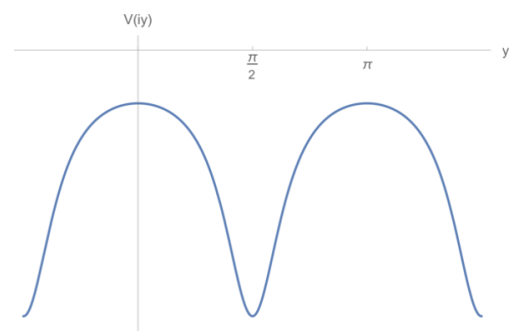


exchanged under T-duality, which in the Euclidean black hole background eq. (3.2a) inverts the metric coefficient $\tanh ^{2}(r) \rightarrow \operatorname{coth}^{2}(r)$ of the compact direction, which is equivalent to a complex field redefinition $r \rightarrow r+\frac{\pi i}{2}$ [3]. Since the saddle-point expansion already requires that we complexify $r$, one obtains a very similar calculation for the pure-momentum modes as for the pure-winding modes.

From eq. (3.31), inserting $\mathcal{O}_{j, n, w=0}$ in the far past on the cylinder imposes the asymptotic conditions

$$
\begin{aligned}
& r(\rho, \phi) \stackrel{\rho \rightarrow-\infty}{\longrightarrow}-\frac{2}{k}\left(j-\frac{1}{2}\right) \rho+\mathcal{O}(1) \\
& \theta(\rho, \phi) \stackrel{\rho \rightarrow-\infty}{\longrightarrow}-\frac{i n}{k} \rho+\mathcal{O}(1),
\end{aligned}
$$

for $\operatorname{Re}(j)>\frac{1}{2}$, which may be extended to complex branch operators by including a regulator $j \rightarrow j+\varepsilon$. From eq. (3.32), the action describing the two-point function of $\mathcal{O}_{j, n, w=0}$ and $\mathcal{O}_{j,-n, w=0}$ is

$$
\begin{aligned}
S_{j n}= & \frac{k}{4 \pi} \int_{-L}^{L} \mathrm{~d} \rho \int_{0}^{2 \pi} \mathrm{d} \phi\left(\left(\partial_{\rho} r\right)^{2}+\left(\partial_{\phi} r\right)^{2}+\tanh ^{2}(r)\left(\left(\partial_{\rho} \theta\right)^{2}+\left(\partial_{\phi} \theta\right)^{2}\right)\right) \\
& +2\left(\frac{1}{2}-j\right) \int_{0}^{2 \pi} \frac{\mathrm{d} \phi}{2 \pi}\left(\left.r\right|_{\rho=L}+\left.r\right|_{\rho=-L}\right)+i n \int_{0}^{2 \pi} \frac{\mathrm{d} \phi}{2 \pi}\left(\left.\theta\right|_{\rho=L}-\left.\theta\right|_{\rho=-L}\right) \\
& +4 \frac{L}{k}\left(j-\frac{1}{2}\right)^{2}-\frac{L}{k} n^{2} .
\end{aligned}
$$

The boundary equations of motion are now

$$
\begin{aligned}
\left.\partial_{\rho} r\right|_{\rho= \pm L} & = \pm \frac{2}{k}\left(j-\frac{1}{2}\right) \\
\left.\tanh ^{2}(r) \partial_{\rho} \theta\right|_{\rho= \pm L} & =-\frac{i n}{k},
\end{aligned}
$$

which, in the large $L$ limit, impose the requisite asymptotic conditions.

In this pure-momentum sector, we look for saddles of the form $r=r(\rho)$ and $\theta=\theta(\rho)$. Then the $\theta$ equation of motion (eq. (3.4b)),

$$
\partial_{\rho}\left(\tanh ^{2}(r) \partial_{\rho} \theta\right)=0
$$

implies that $\tanh ^{2}(r) \partial_{\rho} \theta=-\frac{i n}{k}$ is fixed not only at the boundaries (eq. (4.73b)) but is everywhere constant. Inserting in eq. (4.72) $\partial_{\rho} \theta=-\frac{i n}{k} \operatorname{coth}^{2}(r)$ and its integral

$$
\left.\theta\right|_{\rho=L}-\left.\theta\right|_{\rho=-L}=\int_{-L}^{L} \mathrm{~d} \rho \partial_{\rho} \theta,
$$


we obtain an effective action for $r(\rho)$ :

$$
\begin{aligned}
\frac{1}{k} S_{j n}= & \int_{-L}^{L} \mathrm{~d} \rho\left(\frac{1}{2}\left(\frac{\mathrm{d} r}{\mathrm{~d} \rho}\right)^{2}+\frac{n^{2}}{2 k^{2}} \operatorname{coth}^{2}(r)\right) \\
& +\frac{2}{k}\left(\frac{1}{2}-j\right)\left(\left.r\right|_{\rho=L}+\left.r\right|_{\rho=-L}\right)+\frac{4}{k^{2}}\left(j-\frac{1}{2}\right)^{2} L-\frac{n^{2}}{k^{2}} L .
\end{aligned}
$$

Thus, one finds as expected an effective quantum mechanics in $\operatorname{coth}^{2}(r)$ rather than $\tanh ^{2}(r)$ potential, related to the winding calculation in the preceding sub-sections by $r \rightarrow r+\frac{\pi i}{2}$.

As before, one may then identify the set of saddles that reproduce the large $k$ limit of the exact reflection coefficient, which, at leading order is

$$
\begin{array}{rlrl}
R(\eta, \omega) \stackrel{k \rightarrow \infty}{\longrightarrow} & \eta^{-2 k \eta}(\eta+|\omega|)^{k(\eta+|\omega|)} \csc (\pi k \eta) & \\
& \times \begin{cases}(|\omega|-\eta)^{k(\eta-|\omega|)} & 0<\operatorname{Re}(\eta)<|\omega| \\
(\eta-|\omega|)^{k(\eta-|\omega|)} \sin ^{2}\left(\frac{\pi k}{2}(\eta-|\omega|)\right) & \operatorname{Re}(\eta)>|\omega|,\end{cases}
\end{array}
$$

where $j=\frac{k \eta}{2}$ and $n=k \omega$. As in eq. (4.5), the $\csc (\pi k \eta)$ factor is explained by the complex shift symmetry of the potential. Note the absence in the pure-momentum case of additional csc factors, whose poles in the winding reflection coefficient indicated the existence of the real branch bound states. The bound state spectrum (eq. (3.9b)) is empty for $w=0$, where the associated poles of $R$ are now found on the negative $j$ axis, which is separated from the physical region by Stokes walls, and which would map to non-normalizable wavefunctions (eq. (3.23)). Singular saddles nevertheless appear to be required to account for the $\omega$ dependence of the sin factor for $\operatorname{Re}(\eta)>|\omega|$. It would be interesting to further study the relevance of these singular saddles for scattering in the Lorentzian black hole and to what extent they probe the black hole singularity [39].

\section{Sine-Liouville limit}

The $\operatorname{SL}(2, \mathbb{R})_{k} / \mathrm{U}(1)$ CFT is defined for $k>2$. So far in this note we have focused on the $k \rightarrow \infty$ limit, where the cigar sigma-model provides a weakly-coupled Lagrangian description of the CFT. In the opposite limit, namely $k-2 \rightarrow 0$, the scalar curvature of the cigar diverges, and that description becomes strongly-coupled. However, there exists a dual description of the CFT that is better suited at small $k$ [40, 41]. In this section we consider the saddle-point expansion in the $k-2 \rightarrow 0$ limit using the dual description.

Recall from eq. (3.11) that the cigar sigma-model approaches a free linear-dilaton $\times S^{1}$ background in the weak-coupling region, with canonically normalized coordinates $\hat{r}$ and $\hat{\theta} \sim \hat{\theta}+2 \pi \sqrt{\alpha^{\prime} k}$. The asymptotically linear-dilaton is $\Phi(\hat{r})=-Q \hat{r}$, where $^{33}$

$$
Q=\frac{1}{\sqrt{\alpha^{\prime}(k-2)}} \text {. }
$$

\footnotetext{
${ }^{33}$ Since we are no longer working in the large $k$ limit, here we use the exact value of $Q$, compared to $Q \stackrel{k \rightarrow \infty}{\longrightarrow} \frac{1}{\sqrt{\alpha^{\prime} k}}$ in eq. (3.12). See also footnote 14 .
} 


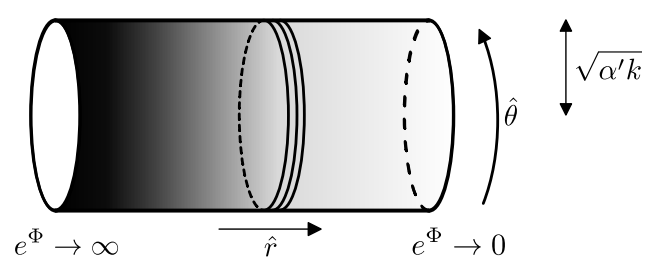

Figure 14. The sine-Liouville background. According to the FZZ duality, the sine-Liouville sigma-model is a dual description of the $\mathrm{SL}(2, \mathbb{R})_{k} / \mathrm{U}(1)$ CFT, better suited when $k-2$ is small. The geometry is an infinite cylinder of radius $\sqrt{\alpha^{\prime} k}$. The dilaton is $\Phi=-Q \hat{r}$, so that the string coupling $e^{\Phi}$ diverges as $\hat{r} \rightarrow-\infty$ and vanishes as $\hat{r} \rightarrow \infty$. The sine-Liouville potential $e^{-\sqrt{(k-2) / \alpha^{\prime}} \hat{r}} \operatorname{Re} e^{i \sqrt{k / \alpha^{\prime}}\left(\hat{\theta}_{\mathrm{L}}-\hat{\theta}_{\mathrm{R}}\right)}$ includes a pure-winding mode of $\hat{\theta}$ (represented by the circles wrapping the middle of the cylinder), times a linear-dilaton primary (represented by the color gradient). Alternatively, one may T-dualize the geometry to obtain a cylinder of radius $\sqrt{\alpha^{\prime} / k}$. We denote the angular coordinates before and after the T-duality by $\hat{\theta}$ and $\hat{\vartheta}$.

The linear-dilaton $\times S^{1}$ itself, with $\hat{r} \in(-\infty, \infty)$ permitted to range over the entire line, is ill-defined because the string coupling diverges as $\hat{r} \rightarrow-\infty$. This strong coupling region is eliminated in the cigar background by ending the geometry. In the dual description, the cigar is replaced by a fully infinite linear-dilaton $\times S^{1}$ background, deformed by the "sineLiouville" potential $V_{\mathrm{SL}} \propto e^{-2 b_{\mathrm{sL}} \hat{r}} \operatorname{Re} e^{i \sqrt{\frac{k}{\alpha^{\prime}}\left(\hat{\theta}_{\mathrm{L}}-\hat{\theta}_{\mathrm{R}}\right)}}$. The potential consists of a Liouville-like radial factor $e^{-2 b_{\mathrm{SL}} \hat{r}}$, together with the unit-winding operator around the $S^{1}$ direction. The linear-dilaton momentum,

$$
b_{\mathrm{sL}}=\frac{1}{2} \sqrt{\frac{k-2}{\alpha^{\prime}}},
$$

is chosen such that the potential is of weight $(1,1)$ :

$$
\alpha^{\prime} b_{\mathrm{sL}}\left(Q-b_{\mathrm{sL}}\right)+\frac{k}{4}=1 .
$$

At large $\hat{r}$, the potential decays and one recovers the same asymptotic linear-dilaton $\times S^{1}$ theory as for the cigar. Note that the presence of the winding operator in the sine-Liouville potential explicitly breaks the winding number symmetry around the cylinder of the free theory. Likewise, the apparent winding conservation law in the asymptotic region of the cigar is violated in the interior, where the string can unwind at the tip. One thinks of the sine-Liouville background as being built up of a condensate of winding strings on top of the cylinder, as pictured in figure 14. The equivalence of the sine-Liouville and cigar descriptions of the $\mathrm{SL}(2, \mathbb{R})_{k} / \mathrm{U}(1) \mathrm{CFT}$ is known as the FZZ duality [40, 41].

In light of the winding operator in the sine-Liouville potential, the background is better written in terms of the T-dual coordinate $\hat{\vartheta} \sim \hat{\vartheta}+2 \pi \sqrt{\frac{\alpha^{\prime}}{k}}$. The action on a closed worldsheet $\Sigma$ is

$$
\begin{aligned}
S= & \frac{1}{4 \pi \alpha^{\prime}} \int_{\Sigma} \mathrm{d}^{2} \sigma \sqrt{h}\left((\nabla \hat{r})^{2}+(\nabla \hat{\vartheta})^{2}+4 \pi \lambda e^{-2 b_{\mathrm{sL}} \hat{r}} \cos \left(\sqrt{\frac{k}{\alpha^{\prime}}} \hat{\vartheta}\right)\right) \\
& -\frac{Q}{4 \pi} \int_{\Sigma} \mathrm{d}^{2} \sigma \sqrt{h} \mathcal{R}[h] \hat{r} .
\end{aligned}
$$


The coefficient $\lambda$ is a positive number, analogous to the coefficient $\mu$ of the Liouville potential (cf. eq. (2.22)). One is again free to add a constant mode to the dilaton, but it may be eliminated by shifting $\hat{r}$ and rescaling $\lambda$.

As in Liouville, the linear-dilaton factor of the potential $e^{-2 b_{\mathrm{sL}} \hat{r}}$ is weakly-coupled when $b_{\mathrm{sL}}$ is small, i.e. when $k$ is near 2 . However, neither the original cylinder radius $\sqrt{\alpha^{\prime} k}$ nor its T-dual $\sqrt{\alpha^{\prime} / k}$ is large in that limit, and so the sine-Liouville background is not strictly speaking weakly-coupled there. It is a far better description of the coset for $k$ near 2 than the cigar, however, which becomes infinitely strongly-coupled in the limit.

Because the asymptotic conditions for the coset operators discussed in section 3.3 in the cigar description mapped the neighborhood of the insertion to the free-field region where the cigar and sine-Liouville backgrounds coincide, the same apply in sine-Liouville. T-dualizing eq. (3.31), the asymptotic conditions for an insertion of $\mathcal{O}_{j n w}$ in the far past on the cylinder are

$$
\begin{aligned}
& \hat{r}(\rho, \phi) \stackrel{\rho \rightarrow-\infty}{\longrightarrow} 2 \alpha^{\prime} Q\left(\frac{1}{2}-j\right) \rho+\mathcal{O}(1) \\
& \hat{\vartheta}(\rho, \phi) \stackrel{\rho \rightarrow-\infty}{\longrightarrow} i w \sqrt{\alpha^{\prime} k} \rho+n \sqrt{\frac{\alpha^{\prime}}{k}} \phi+\mathcal{O}(1) .
\end{aligned}
$$

In the previous discussion on the cigar, the (asymptotically) linear-dilaton played little role as $k \rightarrow \infty$ because $Q$ vanished in the limit. By contrast, $Q$ diverges as $k-2 \rightarrow 0$. The background-charge operators, responsible for the shift by $\frac{1}{2}$ in eq. (5.5a), now behave as heavy operators, scaling with the leading-order terms in the action. Similarly, the $\mathcal{O}_{j n w}$ insertion is itself a heavy operator for $j$ of order one in the $k-2 \rightarrow 0$ limit.

The action with insertions of $\mathcal{O}_{j n w}$ in the far past and $\mathcal{O}_{j,-n,-w}$ in the far future is

$$
\begin{aligned}
S_{j n w}= & \frac{1}{4 \pi \alpha^{\prime}} \int_{-L}^{L} \mathrm{~d} \rho \int_{0}^{2 \pi} \mathrm{d} \phi\left(\left(\partial_{\rho} \hat{r}\right)^{2}+\left(\partial_{\phi} \hat{r}\right)^{2}+\left(\partial_{\rho} \hat{\vartheta}\right)^{2}+\left(\partial_{\phi} \hat{\vartheta}\right)^{2}+4 \pi \lambda e^{-2 b_{\mathrm{sL}} \hat{r}} \cos \left(\sqrt{\frac{k}{\alpha^{\prime}}} \hat{\vartheta}\right)\right) \\
& -\frac{2}{\sqrt{\alpha^{\prime}(k-2)}}\left(j-\frac{1}{2}\right) \int_{0}^{2 \pi} \frac{\mathrm{d} \phi}{2 \pi}\left(\left.\hat{r}\right|_{\rho=L}+\left.\hat{r}\right|_{\rho=-L}\right)-i w \sqrt{\frac{k}{\alpha^{\prime}}} \int_{0}^{2 \pi} \frac{\mathrm{d} \phi}{2 \pi}\left(\left.\hat{\vartheta}\right|_{\rho=L}-\left.\hat{\vartheta}\right|_{\rho=-L}\right) \\
& +\int_{0}^{2 \pi} \frac{\mathrm{d} \phi}{2 \pi}\left(\sigma_{+}\left(\left.\partial_{\phi} \hat{\vartheta}\right|_{\rho=L}-n \sqrt{\frac{\alpha^{\prime}}{k}}\right)+\sigma_{-}\left(\left.\partial_{\phi} \hat{\theta}\right|_{\rho=-L}-n \sqrt{\frac{\alpha^{\prime}}{k}}\right)\right) \\
& +\frac{4}{k-2}\left(j-\frac{1}{2}\right)^{2} L-k w^{2} L-\frac{L}{k} n^{2} .
\end{aligned}
$$

Note that the boundary action for $\hat{\vartheta}$ is well-defined because $w \in \mathbb{Z}$.

Let us again restrict our attention to the $n=0$ sector, where the $k-2 \rightarrow 0$ limit of the exact reflection coefficient eq. (4.1) yields

$$
\begin{aligned}
R(j, w) \stackrel{k-2 \rightarrow 0}{\longrightarrow} & 2^{4\left(j-\frac{1}{2}\right)}\left(j-\frac{1}{2}\right) \frac{\gamma(j+w) \gamma(j-w)}{\gamma(2 j)} \\
& \times\left(\frac{e}{2} \frac{k-2}{j-\frac{1}{2}}\right)^{\frac{4}{k-2}\left(j-\frac{1}{2}\right)} \csc \left(\frac{2 \pi}{k-2}\left(j-\frac{1}{2}\right)\right),
\end{aligned}
$$


where $j=\mathcal{O}\left(k^{0}\right)$ and $\operatorname{Re}(j)>\frac{1}{2}$. Note that the second line, which is the dominant contribution, is independent of $w$.

In this limit, the most interesting factor in eq. (4.1) is $\gamma\left(\frac{2 j-1}{k-2}\right)$, which leads to the csc factor of eq. (5.7). The latter arises in the saddle-point expansion from the following shift symmetry of the sine-Liouville potential:

$$
\begin{aligned}
& \hat{r} \rightarrow \hat{r}+\frac{\pi i}{2 b_{\mathrm{sL}}} \\
& \hat{\vartheta} \rightarrow \hat{\vartheta}+\pi \sqrt{\frac{\alpha^{\prime}}{k}},
\end{aligned}
$$

under which the linear-dilaton and compact-boson factors of the potential each transform by a sign. By the same argument as in section 4.2, the functional integral over real $\hat{r}$ diverges and should instead be defined over an appropriate complex cycle. We expect that the cycle will consist of a sum of steepest-descent contours associated to saddles related by the shift symmetry. Under the shift, the action changes by

$$
S \rightarrow S-\frac{4 \pi i}{k-2}\left(j-\frac{1}{2}\right)
$$

due to the boundary terms. Summing over this discrete moduli space will contribute

$$
\sum_{N \in \mathbb{Z}_{\geq 0}} e^{\frac{4 \pi i}{k-2}\left(j-\frac{1}{2}\right) N}=\frac{i}{2} e^{-\frac{2 \pi i}{k-2}\left(j-\frac{1}{2}\right)} \csc \left(\frac{2 \pi}{k-2}\left(j-\frac{1}{2}\right)\right)
$$

for $\operatorname{Im}(j)>0$, reproducing the csc in eq. (5.7). For $\operatorname{Im}(j)<0$, one sums over $N \in \mathbb{Z}_{\leq 0}$.

Because the sine-Liouville Lagrangian is not actually weakly-coupled, it is more challenging to reproduce the rest of eq. (5.7) by the saddle-point expansion. To attempt to extract the $\frac{1}{k-2}$ scaling from the action, one would define

$$
\tilde{r}=\sqrt{\frac{k-2}{\alpha^{\prime}}} \hat{r}, \quad \tilde{\vartheta}=\sqrt{\frac{k-2}{\alpha^{\prime}}} \hat{\vartheta}, \quad \tilde{\lambda}=\frac{k-2}{\alpha^{\prime}} \lambda,
$$

in terms of which

$$
\begin{aligned}
S_{j w}=\frac{1}{k-2}\{ & \frac{1}{4 \pi} \int_{-L}^{L} \mathrm{~d} \rho \int_{0}^{2 \pi} \mathrm{d} \phi\left(\left(\partial_{\rho} \tilde{r}\right)^{2}+\left(\partial_{\phi} \tilde{r}\right)^{2}+\left(\partial_{\rho} \tilde{\vartheta}\right)^{2}+\left(\partial_{\phi} \tilde{\vartheta}\right)^{2}+4 \pi \tilde{\lambda} e^{-\tilde{r}} \cos \left(\sqrt{\frac{k}{k-2} \tilde{\vartheta}}\right)\right) \\
& -2\left(j-\frac{1}{2}\right) \int_{0}^{2 \pi} \frac{\mathrm{d} \phi}{2 \pi}\left(\left.\tilde{r}\right|_{\rho=L}+\left.\tilde{r}\right|_{\rho=-L}\right)-i w \sqrt{k(k-2)} \int_{0}^{2 \pi} \frac{\mathrm{d} \phi}{2 \pi}\left(\left.\tilde{\vartheta}\right|_{\rho=L}-\left.\tilde{\vartheta}\right|_{\rho=-L}\right) \\
& \left.+4\left(j-\frac{1}{2}\right)^{2} L-k(k-2) w^{2} L\right\} .
\end{aligned}
$$

Were in the functional in braces $\mathcal{O}\left((k-2)^{0}\right)$, one could proceed with the saddle-point expansion as in the preceding sections. However, the sine-Liouville potential oscillates rapidly in this limit, reflecting the fact that the description is not weakly-coupled. 
We will not attempt to reproduce the rest of the semi-classical limit using the sineLiouville description. We point out, however, that the second line of eq. (5.7) coincides with the leading terms in the semi-classical limit of the Liouville reflection coefficient. It was shown in [42] that winding-preserving $n$-point functions in the $\operatorname{SL}(2, \mathbb{R})_{k} / \mathrm{U}(1) \mathrm{CFT}$ are reproduced by a sum of $2 n-2$ point correlation functions in Liouville. In particular, the two-point function of the coset is simply related to the two-point function of Liouville, with a certain dictionary described in [42], and one correspondingly finds that their semiclassical limits are closely related.

\section{Discussion}

In this paper, we have applied saddle-point methods to understand the semi-classical limit of the two-dimensional Euclidean black hole CFT SL $(2, \mathbb{R})_{k} / \mathrm{U}(1)$. Our original motivation in undertaking this work was to discover the asymptotic condition eq. (4.67) that describes an insertion of the sine-Liouville operator in the large $k$ cigar description of the CFT, which plays a role in the Lorentzian continuation of the FZZ duality that we construct in [34] as an example of an ER = EPR string duality. In exploring that question, however, we found that understanding the semi-classical limit of the $\mathrm{SL}(2, \mathbb{R})_{k} / \mathrm{U}(1)$ CFT is itself a rich problem that illustrates remarkable features of the application of complex saddle-point methods to the asymptotic expansions of functional integrals, as well as to black hole physics.

The main result of this paper is the demonstration that there exists a set of complex trajectories on which the leading saddle-point expansion reproduces the large $k$ limit of the exact reflection coefficient of $\mathrm{SL}(2, \mathbb{R})_{k} / \mathrm{U}(1)$. An important feature of the quantum black hole is the existence of the winding string bound states, which manifest as poles of the reflection coefficient. These poles are invisible in perturbation theory and appear only after summing over instantons. We have shown that the contributing instantons necessarily include trajectories that hit the singularities of the black hole. Though singular, these configurations have finite action due to a cancellation between divergences in the real and imaginary parts of the squared speed, enabling a principal-value type definition of the action integral. Because the action is finite, higher order quantum corrections remain suppressed by inverse powers of $k$, and we expect that the semi-classical approximation remains reliable. Alternatively, the contributing trajectories live in different topological sectors of the complexified field space with various winding numbers around the singular points, and the on-shell action may be evaluated as a contour integral that avoids the singularities. Singular configurations also played an important role in the complex saddle-point analyses of $[1,18]$.

We have not attempted to derive this list of saddles from first principles, but rather reverse-engineered it from knowledge of the semi-classical limit of the exact result. It remains an important problem to develop machinery that enables an efficient determination of the set of contributing saddles. This question arises already at the level of the saddlepoint expansion of the path integral for the zero-mode quantum mechanics.

Our analysis here was confined to the leading $e^{\mathcal{O}(k)}$ contribution to the saddle-point expansion. It would also be very interesting to compute the order one quantum correction. The simple, factorized form of the large $k$ limit of the exact reflection coefficient into an 


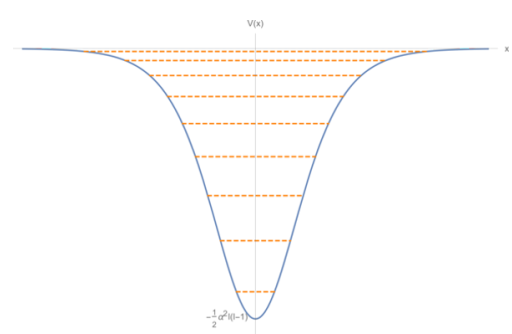

Figure 15. The cigar quantum mechanics potential.

$e^{\mathcal{O}(k)}$ factor times an order one factor indicates that these quantum corrections are the same for all the contributing saddles, up to relative signs as in e.g. eq. (4.44).

\section{Acknowledgments}

We would like to thank Juan Maldacena, Edward Witten, and Xi Yin for stimulating and helpful discussions. This work was supported in part by NSFCAREER grant PHY-1352084.

\section{A Exact solution of the cigar quantum mechanics}

In section 4.2 we saw that the large $k$ limit of the cigar action evaluated on a pure-winding solution $\theta=-w \phi$ reduced to a quantum mechanics for $r$ with a potential proportional to $\operatorname{sech}^{2}(r)$. This quantum mechanics is exactly solvable, ${ }^{34}$ as we review in this appendix.

The potential is usually written in the form

$$
V(x)=-\frac{1}{2} \alpha^{2} l(l-1) \operatorname{sech}^{2}(\alpha x)
$$

with $\alpha>0$ and $l>1$. It is a symmetric well of depth $\frac{1}{2} \alpha^{2} l(l-1)$, and it vanishes as $x \rightarrow \pm \infty$, as pictured in figure 15. It therefore admits both bound states and scattering states. To begin we consider the quantum mechanics on an infinite line, $x \in \mathbb{R}$. The cigar is related to its $\mathrm{Z}_{2}$ quotient $x \sim-x$.

Consider first the scattering states. We look for solutions of

$$
-\frac{1}{2} \psi^{\prime \prime}(x)+V(x) \psi(x)=\frac{p^{2}}{2} \psi(x),
$$

behaving asymptotically as

$$
\psi(x) \rightarrow \begin{cases}e^{i p x}+R(p) e^{-i p x} & x \rightarrow-\infty \\ T(p) e^{i p x} & x \rightarrow \infty\end{cases}
$$

\footnotetext{
${ }^{34}$ See, for example, Landau and Lifshitz's Quantum Mechanics (Second Edition), sections 23 and 25. We set $\hbar$ and the mass to one. The potential is often referred to as the modified Pöschl-Teller potential.
} 
The two linearly independent solutions of this equation are the associated Legendre polynomials $P_{l-1}^{i p / \alpha}(\tanh (\alpha x))$ and $Q_{l-1}^{i p / \alpha}(\tanh (\alpha x))$. The asymptotics of the $P$ function are

$$
\begin{aligned}
& P_{l-1}^{i p / \alpha}(\tanh (\alpha x)) \\
& \quad \rightarrow \begin{cases}\frac{i \pi \operatorname{csch}\left(\frac{\pi p}{\alpha}\right)}{\Gamma\left(1+\frac{i p}{\alpha}\right) \Gamma\left(l-\frac{i p}{\alpha}\right) \Gamma\left(1-l-\frac{i p}{\alpha}\right)} e^{i p x}-\frac{i \sin (\pi l) \operatorname{csch}\left(\frac{\pi p}{\alpha}\right)}{\Gamma\left(1-\frac{i p}{\alpha}\right)} e^{-i p x} & x \rightarrow-\infty \\
\frac{1}{\Gamma\left(1-\frac{i p}{\alpha}\right)} e^{i p x} & x \rightarrow \infty .\end{cases}
\end{aligned}
$$

The asymptotics of the $Q$ function, on the other hand, contain $e^{i p x}$ and $e^{-i p x}$ at both limits, and must be discarded. The scattering wavefunction is then

$$
\psi(x ; p)=-\frac{i}{\pi} \sinh \left(\frac{\pi p}{\alpha}\right) \Gamma\left(1+\frac{i p}{\alpha}\right) \Gamma\left(l-\frac{i p}{\alpha}\right) \Gamma\left(1-l-\frac{i p}{\alpha}\right) P_{l-1}^{i p / \alpha}(\tanh (\alpha x)),
$$

yielding the reflection and transmission coefficients

$$
R(p)=-\frac{1}{\pi} \sin (\pi l) \frac{\Gamma\left(1+\frac{i p}{\alpha}\right)}{\Gamma\left(1-\frac{i p}{\alpha}\right)} \Gamma\left(l-\frac{i p}{\alpha}\right) \Gamma\left(1-l-\frac{i p}{\alpha}\right)
$$

and

$$
T(p)=-\frac{i}{\pi} \sinh \left(\frac{\pi p}{\alpha}\right) \frac{\Gamma\left(1+\frac{i p}{\alpha}\right)}{\Gamma\left(1-\frac{i p}{\alpha}\right)} \Gamma\left(l-\frac{i p}{\alpha}\right) \Gamma\left(1-l-\frac{i p}{\alpha}\right) .
$$

Note this potential has the remarkable property that it is reflectionless when $l$ is an integer; $R(p)$ vanishes due to the factor of $\sin (\pi l)$. In that case, the transmission coefficient may be written

$$
\left.T(p)\right|_{l \in \mathbb{Z}}=\prod_{n=1}^{l-1} \frac{l-n-\frac{i p}{\alpha}}{n-l-\frac{i p}{\alpha}}
$$

by repeatedly applying the factorial property of the Gamma function, $\Gamma(z+1)=z \Gamma(z)$. In particular, $T(p)$ is a pure phase,

$$
\left.|T(p)|^{2}\right|_{l \in \mathbb{Z}}=\prod_{n=1}^{l-1} \frac{(l-n)^{2}+\frac{p^{2}}{\alpha^{2}}}{(n-l)^{2}+\frac{p^{2}}{\alpha^{2}}}=1,
$$

as required by probability conservation, $|R|^{2}+|T|^{2}=1$.

Meanwhile, the bound states are solutions of

$$
-\frac{1}{2} \psi^{\prime \prime}(x)+V(x) \psi(x)=E \psi(x),
$$

with $-\frac{1}{2} \alpha^{2} l(l-1)<E<0$. They may be obtained from the scattering solutions by continuing $p=i \sqrt{2|E|} \in i \mathbb{R}_{+}$, so that $\psi(x ; i \sqrt{2|E|}) \stackrel{x \rightarrow \infty}{\longrightarrow} T e^{-\sqrt{2|E|} x}$ decays. As $x \rightarrow-\infty$,

$$
\psi(x ; i \sqrt{2|E|}) \stackrel{x \rightarrow-\infty}{\longrightarrow} e^{-\sqrt{2|E|} x}+R e^{\sqrt{2|E| x}},
$$


which generically diverges, unless $p=i \sqrt{2|E|}$ is such that $R$ has a pole. At those discrete points, one may hope to find a normalizable bound state proportional to $\frac{1}{R} \psi(x ; i \sqrt{2|E|})$.

$R(p)$ has three sets of simple poles due to the three Gamma functions in its numerator. The first, $\Gamma\left(1+\frac{i p}{\alpha}\right)$, has poles for $p=i \alpha(n+1)$, with $n$ a natural number. These do not correspond to bound states, however, because in the $x \rightarrow-\infty$ limit of the $P$ function it is the ratio $\operatorname{csch}\left(\frac{\pi p}{\alpha}\right) / \Gamma\left(1+\frac{i p}{\alpha}\right)$ that appears, which is regular. The second Gamma function in $R(p), \Gamma\left(l-\frac{i p}{\alpha}\right)$, has poles for $p=-i \alpha(l+n)$, but these do not belong to the domain $i \mathbb{R}_{+}$, which was necessary for convergence at large $x$.

It is instead the last Gamma function which is responsible for the bound states, $\Gamma\left(1-l-\frac{i p}{\alpha}\right)$. The poles are found at

$$
p_{n}=i \alpha(l-1-n)
$$

which belong to $i \mathbb{R}_{+}$provided $n<l-1$. Thus we find the spectrum of bound state energies

$$
E_{n}=\frac{p_{n}^{2}}{2}=-\frac{\alpha^{2}}{2}(l-1-n), \quad 0 \leq n<l-1,
$$

with wavefunctions

$$
\psi_{n}(x)=P_{l-1}^{-(l-1-n)}(\tanh (\alpha x)) .
$$

Next consider the semi-classical limit. Define

$$
\tilde{x} \equiv \alpha x, \quad \tilde{l} \equiv \alpha^{2} \sqrt{l(l-1)},
$$

in terms of which the Hamiltonian may be written

$$
H=\frac{1}{\alpha^{2}}\left(\frac{1}{2}\left(\frac{\mathrm{d} \tilde{x}}{\mathrm{~d} t}\right)^{2}+\tilde{V}(\tilde{x})\right)
$$

where

$$
\tilde{V}(\tilde{x}) \equiv-\frac{1}{2} \tilde{l}^{2} \operatorname{sech}^{2}(\tilde{x}) .
$$

Comparing to eq. (4.15), we find the same quantum mechanics as the pure-winding sector of the cigar CFT, with the dictionary $w=\tilde{l}$ and $k=\frac{1}{\alpha^{2}}$.

The semi-classical limit is $\alpha \rightarrow 0$ with $\tilde{l}$ fixed. In this limit the bound state spectrum is

$$
E_{n} \rightarrow-\frac{\alpha^{2}}{2}\left(n-\frac{\tilde{l}}{\alpha^{2}}\right)^{2} .
$$

The same semi-classical spectrum may be obtained from the WKB approximation, which says that

$$
\int_{-x_{*}}^{x_{*}} \mathrm{~d} x \sqrt{2\left(E_{n}-V(x)\right)}=\pi n
$$

where

$$
x_{*}=\frac{1}{\alpha} \cosh ^{-1}\left(\sqrt{\frac{\frac{1}{2} \alpha^{2} l(l-1)}{-E_{n}}}\right)
$$


is the classical turning point, $V\left( \pm x_{*}\right)=E_{n}$. The integral is

$$
\int_{-x_{*}}^{x_{*}} \mathrm{~d} x \sqrt{2\left(E_{n}-V(x)\right)}=\pi\left(\sqrt{l(l-1)}-\frac{1}{\alpha} \sqrt{-2 E_{n}}\right),
$$

from which we obtain

$$
E_{n} \approx-\frac{\alpha^{2}}{2}(n-\sqrt{l(l-1)})^{2}
$$

reproducing the semi-classical limit of the exact spectrum.

As for the scattering states, define

$$
p \equiv i \frac{\eta}{\alpha}
$$

in terms of which the exact reflection and transmission coefficients may be written

$$
R(\eta)=\frac{1}{\pi} \frac{\tilde{l}-\eta}{\eta} \frac{\sin (\pi k \tilde{l})}{\gamma(k \eta)} \Gamma(k(\tilde{l}+\eta)) \Gamma(-k(\tilde{l}-\eta))
$$

and

$$
T(\eta)=-\frac{1}{\pi} \frac{\tilde{l}-\eta}{\eta} \frac{\sin (\pi k \eta)}{\gamma(k \eta)} \Gamma(k(\tilde{l}+\eta)) \Gamma(-k(\tilde{l}-\eta)),
$$

where again $k \equiv \frac{1}{\alpha^{2}}$. Applying eqs. (4.3)-(4.4) we find in the semi-classical limit

$$
\begin{aligned}
& R(\eta) \stackrel{k \rightarrow \infty}{\longrightarrow} \eta^{-2 k \eta}(\tilde{l}+\eta)^{k(\tilde{l}+\eta)} \sin (\pi k \tilde{l}) \csc (\pi k \eta) \\
& \times \begin{cases}-\frac{1}{2}(\tilde{l}-\eta)^{k(\eta-\tilde{l})} \csc (\pi k(\tilde{l}-\eta)) & 0<\operatorname{Re}(\eta)<\tilde{l} \\
-i(\eta-\tilde{l})^{k(\eta-\tilde{l})} & \operatorname{Re}(\eta)>\tilde{l}\end{cases} \\
& \times \sqrt{\frac{\tilde{l}-\eta}{\tilde{l}+\eta}}
\end{aligned}
$$

and

$$
\begin{aligned}
& T(\eta) \stackrel{k \rightarrow \infty}{\longrightarrow} \eta^{-2 k \eta}(\tilde{l}+\eta)^{k(\tilde{l}+\eta)} \\
& \times \begin{cases}\frac{1}{2}(\tilde{l}-\eta)^{k(\eta-\tilde{l})} \csc (\pi k(\tilde{l}-\eta)) & 0<\operatorname{Re}(\eta)<\tilde{l} \\
i(\eta-\tilde{l})^{k(\eta-\tilde{l})} & \operatorname{Re}(\eta)>\tilde{l}\end{cases} \\
& \times \sqrt{\frac{\tilde{l}-\eta}{\tilde{l}+\eta}}
\end{aligned}
$$

The bound states now correspond to the poles of the $\csc (\pi k(\tilde{l}-\eta))$ factors.

So far we have considered the quantum mechanics on a fully-infinite line. However, the cigar quantum mechanics obtained in section 4.2 by setting $\theta=-w \phi$ was defined on a half-line. Returning to eq. (A.2), the scattering solutions on a half-line are now the linear combinations of Legendre polynomials that vanish at the origin:

$$
\begin{aligned}
\psi_{1 / 2}(x ; p)= & 2 \frac{\Gamma\left(l-\frac{i p}{\alpha}\right) \Gamma\left(1-l-\frac{i p}{\alpha}\right)}{\Gamma\left(-\frac{i p}{\alpha}\right)} \cos ^{2}\left(\frac{\pi}{2}\left(l+\frac{i p}{\alpha}\right)\right) \\
& \times\left(P_{l-1}^{i p / \alpha}(\tanh (\alpha x))-\frac{2}{\pi} \tan \left(\frac{\pi}{2}\left(l+\frac{i p}{\alpha}\right)\right) Q_{l-1}^{i p / \alpha}(\tanh (\alpha x))\right) .
\end{aligned}
$$


It behaves as

$$
\psi_{1 / 2}(x ; p) \stackrel{x \rightarrow-\infty}{\longrightarrow} e^{i p x}+R_{1 / 2}(p) e^{-i p x},
$$

where the reflection coefficient for the half-line problem is

$$
\begin{aligned}
R_{1 / 2}(p)= & 2 \frac{\Gamma\left(l-\frac{i p}{\alpha}\right) \Gamma\left(1-l-\frac{i p}{\alpha}\right)}{\Gamma\left(1-\frac{i p}{\alpha}\right) \Gamma\left(-\frac{i p}{\alpha}\right)} \\
& \times \sin \left(\frac{\pi}{2}\left(l-\frac{i p}{\alpha}\right)\right) \cos \left(\frac{\pi}{2}\left(l+\frac{i p}{\alpha}\right)\right) \csc \left(\frac{\pi i p}{\alpha}\right) .
\end{aligned}
$$

Alternatively, having already solved the theory on a line, the solution on the half-line is given by its quotient with respect to the reflection symmetry $x \sim-x$. The reflection coefficient $R_{1 / 2}(p)$ is then the difference of the reflection and transmission coefficients $R(p)$ and $T(p)$,

$$
R_{1 / 2}(p)=R(p)-T(p),
$$

as can be checked for eqs. (A.6), (A.7), and (A.30), and the bound state spectrum is given by the odd solutions

$$
E_{n}=-\frac{\alpha^{2}}{2}(l-1-n)^{2}, \quad n=1,3,5, \ldots<l-1 .
$$

Previously, we identified the bound states with the poles $p_{n}=i \alpha(l-1-n)$ of $\Gamma\left(1-l-\frac{i p}{\alpha}\right)$. Now we find

$$
R_{1 / 2}\left(p_{n}\right)=\frac{\Gamma(2 l-1-n)}{\Gamma(l-n) \Gamma(l-1-n)} \Gamma(-n)\left((-)^{n}-1\right) .
$$

Whereas $R\left(p_{n}\right) \supset \Gamma(-n)$ was singular for all $n=0,1,2, \ldots$, the additional factor of $(-)^{n}-1$ in $R_{1 / 2}\left(p_{n}\right)$ eliminates the poles with even $n$.

Finally, let us compute the semi-classical limit of $R_{1 / 2}$. With the same notation as before we may write

$$
\begin{aligned}
R_{1 / 2}(\eta)= & 2 \frac{\tilde{l}-\eta}{\eta} \frac{\Gamma(k(\tilde{l}+\eta)) \Gamma(-k(\tilde{l}-\eta))}{\Gamma(k \eta)^{2}} \\
& \times \sin \left(\frac{\pi}{2} k(\tilde{l}+\eta)\right) \cos \left(\frac{\pi}{2} k(\tilde{l}-\eta)\right) \csc (\pi k \eta) .
\end{aligned}
$$

At large $k$ we obtain

$$
\begin{aligned}
R_{1 / 2}(\eta) \stackrel{k \rightarrow \infty}{\longrightarrow} & \eta^{-2 k \eta}(\tilde{l}+\eta)^{k(\tilde{l}+\eta)} \csc (\pi k \eta) \sin \left(\frac{\pi}{2} k(\tilde{l}+\eta)\right) \\
& \times\left\{\begin{array}{l}
-\frac{1}{2}(\tilde{l}-\eta)^{k(\eta-\tilde{l})} \csc \left(\frac{\pi}{2} k(\tilde{l}-\eta)\right) \quad 0<\operatorname{Re}(\eta)<\tilde{l} \\
-2 i(\eta-\tilde{l})^{k(\eta-\tilde{l})} \cos \left(\frac{\pi}{2} k(\tilde{l}-\eta)\right) \quad \operatorname{Re}(\eta)>\tilde{l}
\end{array}\right. \\
& \times \sqrt{\frac{\tilde{l}-\eta}{\tilde{l}+\eta}} .
\end{aligned}
$$


Compared to eq. (A.26), the factor of $\csc (\pi k(\tilde{l}-\eta))$ has been replaced by $\csc \left(\frac{\pi}{2} k(\tilde{l}-\eta)\right)$, reflecting the smaller set of bound states.

Looking back at the large $k$ limit of the exact coset reflection coefficient eq. (4.5), we find agreement with eq. (A.35) to order $e^{k}$, where the discrepancy between $\cos \left(\frac{\pi}{2} k(\tilde{l}-\eta)\right)$ and $\sin \left(\frac{\pi}{2} k(\tilde{l}-\eta)\right)$ in those expressions is of order $e^{k^{0}}$. We conclude that the restriction to the cigar quantum mechanics in section 4.2 is sufficient to extract the saddle-point expansion of the reflection coefficient, i.e. $\theta=-w \phi$ is the only saddle of the $\theta$ equations of motion that contributes to the expansion.

Open Access. This article is distributed under the terms of the Creative Commons Attribution License (CC-BY 4.0), which permits any use, distribution and reproduction in any medium, provided the original author(s) and source are credited.

\section{References}

[1] D. Harlow, J. Maltz and E. Witten, Analytic continuation of Liouville theory, JHEP 12 (2011) 071 [arXiv: 1108.4417] [INSPIRE].

[2] E. Witten, On string theory and black holes, Phys. Rev. D 44 (1991) 314 [InSPIRE].

[3] R. Dijkgraaf, H.L. Verlinde and E.P. Verlinde, String propagation in a black hole geometry, Nucl. Phys. B 371 (1992) 269 [InSPIRE].

[4] J.M. Maldacena and H. Ooguri, Strings in $A d S_{3}$ and $\mathrm{SL}(2, R)$ WZW model. Part 1. The spectrum, J. Math. Phys. 42 (2001) 2929 [hep-th/0001053] [INSPIRE].

[5] J.M. Maldacena, H. Ooguri and J. Son, Strings in $A d S_{3}$ and the $\mathrm{SL}(2, R)$ WZW model. Part 2. Euclidean black hole, J. Math. Phys. 42 (2001) 2961 [hep-th/0005183] [INSPIRE].

[6] J.M. Maldacena and H. Ooguri, Strings in $A d S_{3}$ and the $\mathrm{SL}(2, R)$ WZW model. Part 3. Correlation functions, Phys. Rev. D 65 (2002) 106006 [hep-th/0111180] [INSPIRE].

[7] K. Gawędzki, Noncompact WZW conformal field theories, in NATO advanced study institute: new symmetry principles in quantum field theory, (1991), pg. 0247 [hep-th/9110076] [INSPIRE].

[8] J. Teschner, On structure constants and fusion rules in the $\mathrm{SL}(2, C) / \mathrm{SU}(2)$ WZNW model, Nucl. Phys. B 546 (1999) 390 [hep-th/9712256] [INSPIRE].

[9] J. Teschner, Operator product expansion and factorization in the $H_{3}^{+}-W Z N W$ model, Nucl. Phys. B 571 (2000) 555 [hep-th/9906215] [INSPIRE].

[10] A. Hanany, N. Prezas and J. Troost, The partition function of the two-dimensional black hole conformal field theory, JHEP 04 (2002) 014 [hep-th/0202129] [INSPIRE].

[11] A. Giveon and D. Kutasov, Comments on double scaled little string theory, JHEP 01 (2000) 023 [hep-th/9911039] [INSPIRE].

[12] E. Witten, A new look at the path integral of quantum mechanics, arXiv:1009.6032 [INSPIRE].

[13] E. Witten, Analytic continuation of Chern-Simons theory, AMS/IP Stud. Adv. Math. 50 (2011) 347 [arXiv:1001.2933] [INSPIRE]. 
[14] R. Balian, G. Parisi and A. Voros, Quartic oscillator, in Feynman path integrals, (1978), pg. 337.

[15] A. Voros, The return of the quartic oscillator. The complex WKB method, Ann. I.H.P. Phys. Théor. 39 (1983) 211.

[16] A. Cherman, D. Dorigoni and M. Ünsal, Decoding perturbation theory using resurgence: Stokes phenomena, new saddle points and Lefschetz thimbles, JHEP 10 (2015) 056 [arXiv: 1403.1277] [INSPIRE].

[17] A. Behtash, G.V. Dunne, T. Schäfer, T. Sulejmanpasic and M. Ünsal, Complexified path integrals, exact saddles and supersymmetry, Phys. Rev. Lett. 116 (2016) 011601 [arXiv: 1510.00978] [INSPIRE].

[18] A. Behtash, G.V. Dunne, T. Schäfer, T. Sulejmanpasic and M. Ünsal, Toward Picard-Lefschetz theory of path integrals, complex saddles and resurgence, Ann. Math. Sci. Appl. 02 (2017) 95 [arXiv:1510.03435] [INSPIRE].

[19] T. Fujimori, S. Kamata, T. Misumi, M. Nitta and N. Sakai, Nonperturbative contributions from complexified solutions in $C P^{N-1}$ models, Phys. Rev. D 94 (2016) 105002 [arXiv: 1607.04205] [INSPIRE].

[20] A. Behtash, G.V. Dunne, T. Schaefer, T. Sulejmanpasic and M. Ünsal, Critical points at infinity, non-gaussian saddles, and bions, JHEP 06 (2018) 068 [arXiv:1803.11533] [INSPIRE].

[21] H. Dorn and H.J. Otto, Two and three point functions in Liouville theory, Nucl. Phys. B 429 (1994) 375 [hep-th/9403141] [INSPIRE].

[22] A.B. Zamolodchikov and A.B. Zamolodchikov, Structure constants and conformal bootstrap in Liouville field theory, Nucl. Phys. B 477 (1996) 577 [hep-th/9506136] [INSPIRE].

[23] N. Seiberg, Notes on quantum Liouville theory and quantum gravity, Prog. Theor. Phys. Suppl. 102 (1990) 319 [INSPIRE].

[24] O. Aharony, A. Giveon and D. Kutasov, LSZ in LST, Nucl. Phys. B 691 (2004) 3 [hep-th/0404016] [INSPIRE].

[25] J.B. Hartle and S.W. Hawking, Path integral derivation of black hole radiance, Phys. Rev. D 13 (1976) 2188 [INSPIRE].

[26] J. Teschner, Liouville theory revisited, Class. Quant. Grav. 18 (2001) R153 [hep-th/0104158] [INSPIRE].

[27] A. Giveon, N. Itzhaki and D. Kutasov, Stringy horizons II, JHEP 10 (2016) 157 [arXiv: 1603.05822] [INSPIRE].

[28] M.V. Berry, Infinitely many Stokes smoothings in the gamma function, Proc. Roy. Soc. 434 (1991) 465.

[29] S. Pasquetti and R. Schiappa, Borel and Stokes nonperturbative phenomena in topological string theory and $c=1$ matrix models, Annales Henri Poincaré 11 (2010) 351 [arXiv: 0907.4082] [INSPIRE].

[30] D. Kutasov, Accelerating branes and the string/black hole transition, hep-th/0509170 [INSPIRE].

[31] A. Giveon, N. Itzhaki and D. Kutasov, Stringy horizons, JHEP 06 (2015) 064 [arXiv: 1502.03633] [INSPIRE]. 
[32] A. Giveon and N. Itzhaki, String theory at the tip of the cigar, JHEP 09 (2013) 079 [arXiv: 1305.4799] [INSPIRE].

[33] G. Giribet, Scattering of low lying states in the black hole atmosphere, Phys. Rev. D 94 (2016) 026008 [Addendum ibid. 94 (2016) 049902] [arXiv: 1606.06919] [INSPIRE].

[34] D.L. Jafferis and E. Schneider, Stringy ER=EPR, arXiv:2104.07233 [INSPIRE].

[35] A. Giveon and N. Itzhaki, Stringy black hole interiors, JHEP 11 (2019) 014 [arXiv: 1908.05000] [INSPIRE].

[36] R. Ben-Israel, A. Giveon, N. Itzhaki and L. Liram, On the black hole interior in string theory, JHEP 05 (2017) 094 [arXiv: 1702.03583] [INSPIRE].

[37] E. Witten, Open strings on the Rindler horizon, JHEP 01 (2019) 126 [arXiv:1810.11912] [INSPIRE].

[38] L. Susskind and J. Uglum, Black hole entropy in canonical quantum gravity and superstring theory, Phys. Rev. D 50 (1994) 2700 [hep-th/9401070] [INSPIRE].

[39] M. Grinberg and J. Maldacena, Proper time to the black hole singularity from thermal one-point functions, JHEP 03 (2021) 131 [arXiv: 2011.01004] [INSPIRE].

[40] V. Fateev, A. Zamolodchikov and Al. Zamolodchikov, unpublished.

[41] V. Kazakov, I.K. Kostov and D. Kutasov, A matrix model for the two-dimensional black hole, Nucl. Phys. B 622 (2002) 141 [hep-th/0101011] [INSPIRE].

[42] S. Ribault and J. Teschner, $H_{3}^{+}-W Z N W$ correlators from Liouville theory, JHEP 06 (2005) 014 [hep-th/0502048] [INSPIRE]. 